\title{
Set Theoretic Rajan Transform and its Properties
}

\author{
By G. Prashanthi, G. Sathya, Manish Prateek \& E. G. Rajan
}

University of Petroleum and Energy Studies

Abstract- In this paper, we describe the formulation of a novel transform called Set Theoretic Rajan Transform (STRT) which is an extension of Rajan Transform (RT). RT is a coding morphism by which a number sequence (integer, rational, real, or complex) of length equal to any power of two is transformed into a highly correlated number sequence of same length. STRT was introduced by G. Sathya. In STRT, RT is applied to a sequence of sets instead of sequences of numbers. Here the union $(U)$ is analogous to addition $(+)$ operation and symmetric difference $(\sim)$ is analogous to subtraction (-). This transform satisfies some interesting set theoretic properties like Cyclic Shift Invariance, Dyadic Shift invariance, Graphical Inverse Invariance. This paper explains in detail about STRT and all of its set theoretic properties.

\section{GJCST-D Classification: F.2.1}

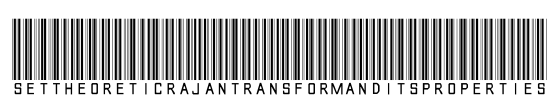

Strictly as per the compliance and regulations of:

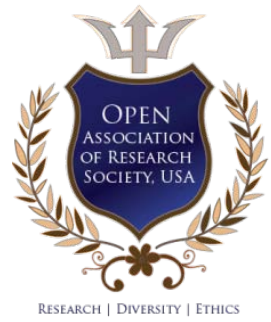

(C) 2020. G. Prashanthi, G. Sathya, Manish Prateek \& E. G. Rajan. This is a research/review paper, distributed under the terms of the Creative Commons Attribution-Noncommercial 3.0 Unported License http://creativecommons.org/licenses/by-nc/3.0/), permitting all non commercial use, distribution, and reproduction in any medium, provided the original work is properly cited. 


\title{
Set Theoretic Rajan Transform and its Properties
}

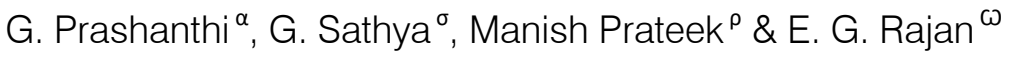

Abstract- In this paper, we describe the formulation of a novel transform called Set Theoretic Rajan Transform (STRT) which is an extension of Rajan Transform (RT). RT is a coding morphism by which a number sequence (integer, rational, real, or complex) of length equal to any power of two is transformed into a highly correlated number sequence of same length. STRT was introduced by G. Sathya. In STRT, RT is applied to a sequence of sets instead of sequences of numbers. Here the union $(U)$ is analogous to addition $(+)$ operation and symmetric difference $(\sim)$ is analogous to subtraction $(-)$. This transform satisfies some interesting set theoretic properties like Cyclic Shift Invariance, Dyadic Shift invariance, Graphical Inverse Invariance. This paper explains in detail about STRT and all of its set theoretic properties.

\section{InTRODUCTION}

n STRT, given a sequence of sets $X(n)$ of length $N$, which is a power of two, first it is divided into the first half and the second half each consisting of (N/2) points so that the following holds good:

$$
\begin{aligned}
& G(j)=X(i) U X(i+(N / 2)) ; 0 \leq j \leq(N / 2) ; 0 \leq i \leq(N / 2) \\
& H(j)=X(i) \sim X(i-(N / 2)) ; \quad 0 \leq j \leq(N / 2) ; 0 \leq i \leq(N / 2)
\end{aligned}
$$

Now each (N/2) -point segment is further divided into two half's each consisting of (N/4) points so that the following holds good:

$$
\begin{array}{ll}
\mathrm{G} 1(\mathrm{k})=\mathrm{G}(\mathrm{j}) \cup \mathrm{G}(\mathrm{j}+(\mathrm{N} / 4)) ; 0 \leq \mathrm{k} \leq(\mathrm{N} / 4) ; 0 \leq \mathrm{j} \leq(\mathrm{N} / 4) \\
\mathrm{G} 2(\mathrm{k})=\mathrm{G}(\mathrm{j}) \sim \mathrm{G}(\mathrm{j}-(\mathrm{N} / 4)) ; 0 \leq \mathrm{k} \leq(\mathrm{N} / 4) ; 0 \leq \mathrm{j} \leq(\mathrm{N} / 4) \\
\mathrm{H} 1(\mathrm{k})=\mathrm{H}(\mathrm{j}) \cup H(\mathrm{j}+(\mathrm{N} / 4)) ; 0 \leq \mathrm{k} \leq(\mathrm{N} / 4) ; 0 \leq \mathrm{j} \leq(\mathrm{N} / 4) \\
\mathrm{H} 2(\mathrm{k})=\mathrm{H}(\mathrm{j}) \sim \mathrm{H}(\mathrm{j}-(\mathrm{N} / 4)) ; 0 \leq \mathrm{k} \leq(\mathrm{N} / 4) ; 0 \leq \mathrm{j} \leq(\mathrm{N} / 4)
\end{array}
$$

This process is continued till no more division is possible. The total number of stages thus turns out to be log2N. Then the signal flow graph for STRT of length eight would be of the form shown in the Fig. 1.

Author a: Director, Computer Vision, Pentagram Research Centre Pvt. Ltd., Hyderabad, India. e-mail: prashanthi89@gmail.com

Author o: Director, Artificial Intelligence, Pentagram Research Centre Pvt. Ltd., Hyderabad, India. e-mail: sathyag413@gmail.com

Author $\rho$ : Professor and Dean, University of Petroleum and Energy Studies, Dehradun Uttarakhand, India.

e-mail:mprateek@ddn.upes.ac.in

Author W: Adjunct Professor, University of Petroleum and Energy Studies, Dehradun Uttarakhand, India. e-mail: dr.rajaneg@gmail.com

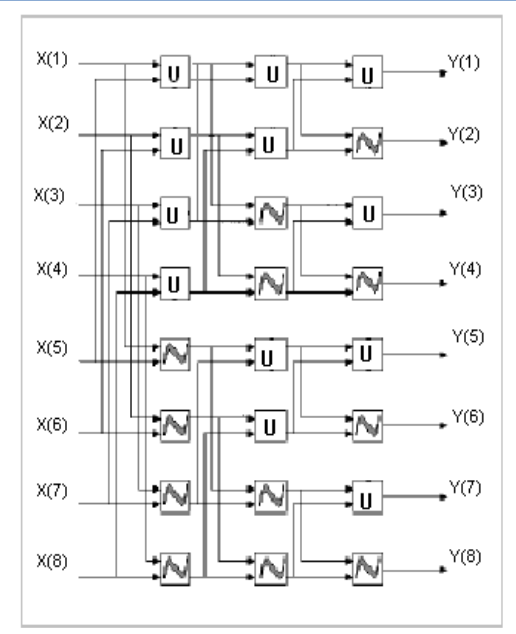

Fig. 1: Signal Flow graph of STRT

Unlike RT, duality doesn't hold good in STRT. If $X(n)$ is a set sequence of length $N=2 k, k>0$ then its Set Theoretic Rajan Transform is denoted by $Y(k)$. Consider a set sequence $X(1)=\{1,2\}, X(2)=\{3,4,6\}, X(3)=\{4,5\}$, $X(4)=\{1,5\}, X(5)=\{1,4,5\}, X(6)=\{3,4,5\}, X(7)=\{2,6\}$, $X(8)=\{1,4,6\}$. Then STRT is computed as follows.

\begin{tabular}{|c|c|c|c|}
\hline $\begin{array}{c}\text { Input set } \\
\text { sequence }\end{array}$ & Stage \#1 & Stage \#2 & $\begin{array}{c}\text { Stage \#3 } \\
\text { STRT } \\
\text { spectrum }\end{array}$ \\
\hline$\{1,2\}$ & $\{1,2,4,5\}$ & $\{1,2,3,4,5,6\}$ & $\{1,2,3,4,5,6\}$ \\
\hline$\{3,4,6\}$ & $\{3,4,5,6\}$ & $\{1,3,4,5,6\}$ & $\{2,3\}$ \\
\hline$\{4,5\}$ & $\{2,4,5,6\}$ & $\{1,6\}$ & $\{1,3,6\}$ \\
\hline$\{1,5\}$ & $\{1,4,5,6\}$ & $\{1,3\}$ & $\{3,6\}$ \\
\hline$\{1,4,5\}$ & $\{2,4,5\}$ & $\{2,4,5,6\}$ & $\{2,4,5,6\}$ \\
\hline$\{3,4,5\}$ & $\{5,6\}$ & $\{4,5,6\}$ & $\{2\}$ \\
\hline$\{2,6\}$ & $\{2,4,5,6\}$ & $\{6\}$ & $\{4,6\}$ \\
\hline$\{1,4,6\}$ & $\{4,5,6\}$ & $\{4\}$ & $\{4,6\}$ \\
\hline
\end{tabular}

\section{il. Algebraic Properties of Strt}

STRT satisfies few interesting properties like Cyclic Shift Invariance, Graphical Inverse Invariance, Dyadic Shift invariance. All these properties are discussed below.

a) Cyclic Shift Invariance

Let us consider the same set of sequences $X(n)=\{1,2\}, \quad\{3,4,6\},\{4,5\}\{1,5\},\{1,4,5\},\{3,4,5\},\{2,6\}$, $\{1,4,6\}$. Using this set of sequences, one can generate seven more cyclic shifted versions such as $X_{C 1}=\{1,4,6\},\{1,2\},\{3,4,6\},\{4,5\}\{1,5\},\{1,4,5\},\{3,4,5\}$, $\{2,6\} ; \quad X c 2=\{2,6\},\{1,4,6\}, \quad\{1,2\},\{3,4,6\},\{4,5\}\{1,5\}$, $\{1,4,5\}, \quad\{3,4,5\} ; \quad X c 3=\{3,4,5\}, \quad\{2,6\},\{1,4,6\},\{1,2\}$, 
$\{3,4,6\},\{4,5\}\{1,5\},\{1,4,5\} ; \quad X_{c} 4=\{1,4,5\},\{3,4,5\},\{2,6\}$, $\{1,4,6\},\{1,2\},\{3,4,6\},\{4,5\}\{1,5\} ; \quad X c 5=\{1,5\},\{1,4,5\}$, $\{3,4,5\},\{2,6\},\{1,4,6\},\{1,2\},\{3,4,6\},\{4,5\} ; \quad X c 6=\{4,5\}$, $\{1,5\},\{1,4,5\},\{3,4,5\},\{2,6\},\{1,4,6\},\{1,2\},\{3,4,6\} ; X_{c} 7=$ $\{3,4,6\},\{4,5\},\{1,5\},\{1,4,5\},\{3,4,5\},\{2,6\},\{1,4,6\},\{1,2\}$. It is obvious that the cyclic shifted version of $X_{c} 7(n)$ is $X(n)$ itself. One can easily verify that all these eight sequences have the same $Y(k)$, that is $\{1,2,3,4,5,6\}$, $\{2,3\},\{1,3,6\},\{3,6\},\{2,4,5,6\},\{2\},\{4,6\},\{4,6\}$.

\section{b) Graphical Inverse Invariance Property}

Consider a sample sequence $X(n)=\{1,2\}$, $\{3,4,6\},\{4,5\}\{1,5\},\{1,4,5\},\{3,4,5\},\{2,6\},\{1,4,6\}$ and its has Graphical Inverse $X-1(n)=\{1,4,6\},\{2,6\},\{3,4,5\}$, $\{1,4,5\},\{1,5\},\{4,5\},\{3,4,6\},\{1,2\}$. Using this sequence one can generate seven more cyclic shifted versions such as Xc1-1(n)=\{1,2\},\{1,4,6\},\{2,6\},\{3,4,5\},\{1,4,5\}, $\{1,5\},\{4,5\},\{3,4,6\} ; \quad X c 2-1(n)=\{3,4,6\},\{1,2\},\{1,4,6\}$, $\{2,6\},\{3,4,5\},\{1,4,5\},\{1,5\},\{4,5\} ; \quad X \subset 3-1(n)=\{4,5\}$, $\{3,4,6\},\{1,2\},\{1,4,6\},\{2,6\},\{3,4,5\},\{1,4,5\},\{1,5\} ; \quad X_{c} 4-$ $1(n)=\{1,5\},\{4,5\},\{3,4,6\},\{1,2\},\{1,4,6\},\{2,6\},\{3,4,5\}$, $\{1,4,5\} ; \quad X c 5-1(n)=\{1,4,5\},\{1,5\},\{4,5\}, \quad\{3,4,6\},\{1,2\}$, $\{1,4,6\},\{2,6\},\{3,4,5\} ; \quad X c 6-1(n)=\{3,4,5\},\{1,4,5\},\{1,5\}$, $\{4,5\},\{3,4,6\},\{1,2\},\{1,4,6\},\{2,6\} ; \quad X \mathrm{C} 7-1(n)=\{2,6\}$ $\{3,4,5\},\{1,4,5\},\{1,5\},\{4,5\}, \quad\{3,4,6\},\{1,2\},\{1,4,6\}$. It is obvious that the cyclic shifted version of $X c 8-1(n)$ is $X$ $1(n)$ itself. One can easily verify that all these eight sequences have the same $Y(k)$, that is, $\{1,2,3,4,5,6\}$, $\{2,3\},\{1,3,6\},\{3,6\},\{2,4,5,6\},\{2\},\{4,6\},\{4,6\}$.

\section{c) Dyadic Shift Invariance property}

The term 'dyad' refers to a group of two, and the term 'dyadic shift' to the operation of transposition of two blocks of elements in a sequence. For instance, let us take $X(n)=\{1,2\},\{3,4,6\},\{4,5\}\{1,5\},\{1,4,5\},\{3,4,5\}$, $\{2,6\},\{1,4,6\}$ and transpose its first half with the second half. The resulting sequence $\operatorname{Td}(2)[X n)]=\{1,4,5\}$, $\{3,4,5\},\{2,6\} .\{1,4,6\},\{1,2\},\{3,4,6\},\{4,5\},\{1,5\}$ is the 2-block dyadic shifted version of $X(n)$. The symbol Td(2) denotes the 2- block dyadic shift operator. In the same manner, we obtain $\operatorname{Td}(4)[\operatorname{Td}(2)[X(n)]]=\{2,6\},\{1,4,6\}$ $\{1,4,5\},\{3,4,5\},\{4,5\},\{1,5\},\{1,2\},\{3,4,6\}$ and $\operatorname{Td}(8)[T d$ (4) $[\operatorname{Td}(2)[X(n)]]]=\{1,4,6\},\{2,6\},\{3,4,5\}, \quad\{1,4,5\},\{1,5\}$, $\{4,5\},\{3,4,6\},\{1,2\}$. One can easily verify that all these dyadic shifted sequences have the same $Y(k)$, that is, $\{1,2,3,4,5,6\},\{2,3\},\{1,3,6\},\{3,6\}, \quad\{2,4,5,6\},\{2\},\{4,6\}$, $\{4,6\}$. There is yet another way of dyadic shifting input sequence $X(n)$ to $\operatorname{Td}(2)[\operatorname{Td}(4)[\operatorname{Td}(8)[X(n)]]]$. Let us take $X(n)=,\{1,2\}, \quad\{3,4,6\},\{4,5\}\{1,5\},\{1,4,5\},\{3,4,5\},\{2,6\}$, $\{1,4,6\}$ and obtain following dyadic shifts: $\operatorname{Td}(8)[X(n)]=$ $\{3,4,6\},\{1,2\},\{1,5\},\{4,5\},\{3,4,5\},\{1,4,5\}, \quad\{1,4,6\},\{2,6\}$ $\operatorname{Td}(4)[\operatorname{Td}(8)[X(n)]]=\{1,5\},\{4,5\},\{3,4,6\}, \quad\{1,2\},\{1,4,6\}$, $\{2,6\},\{3,4,5\},\{1,4,5\}$ and $\operatorname{Td}(2)[\operatorname{Td}(4) \quad[\operatorname{Td}(8)[X(n)]]]=$ $\{1,4,6\},\{2,6\},\{3,4,5\},\{1,4,5\},\{1,5\}, \quad\{4,5\},\{3,4,6\}$, $\{1,2\}$. Note that $\operatorname{Td}(2)[\operatorname{Td}(4)[\operatorname{Td}(8)[X(n)]]]=\operatorname{Td}(8)[\operatorname{Td}(4)$ $[\operatorname{Td}(2)[X(n)]]]$. One can easily verify from the above that other than $\operatorname{Td}(4)[\operatorname{Td}(2)[X(n)]]$ and $\operatorname{Td}(8)[X(n)]$, all other dyadically permuted sequences fall under the category of the cyclic permutation class of $X(n)$ and $X-1(n)$. This amounts to saying that the cyclic permutation class of $X(n)$ has eight non-repeating independent sequences, that of $X-1(n)$ has eight non-repeating independent sequences and the dyadic permutation classes of $X(n)$ has two non-repeating independent sequences. To conclude, all these 18 sequences could be seen to have the same $Y(k)$.Set Theoretic Rajan Transform has many emerging applications. It can be used as a powerful tool in encrypting digital (color) images. It has many other applications in domains like Signal Processing and Higher Order Mathematics.

\section{ili. Application of strt in the Study of Extended Topological Filters Defined Over a Finite Set}

Consider a finite set $X=\{a, b, c\}$. Then its power set is $\{\{\Phi\},\{a\},\{b\},\{c\},\{a, b\},\{a, c\},\{b, c\},\{a, b, c\}\}$. One can construct a filter set $F$ whose elements satisfy the following property: 'Any element of $F$ ensures the presence of all its super sets present in the power set of $X$. For example consider a set $X=\{a, b, c\}$. The power set is $\{\phi,\{a\},\{b\},\{c\},\{a, b\},\{a, c\},\{b, c\},\{a, b, c\}\}$. The set $F=\{\{a\},\{a, b\},\{a, c\},\{a, b, c\}\}$ is a valid topological filter set since every element in $\mathrm{F}$ ensures the presence of all its super sets. One can construct 18 such topological filters from the ground set $X$ as shown in table 1.

Table 1: List of topological filters from $X=\{a, b, c\}$

\begin{tabular}{|c|c|c|}
\hline Filters & Filter Contents & $\begin{array}{c}\text { Cardi } \\
\text { nality }\end{array}$ \\
\hline F1 & $\{\{a\},\{b\},\{c\},\{a, b\},\{a, c\},\{b, c\},\{a, b, c\}$ & 7 \\
\hline F2 & $\{\{a\},\{b\},\{a, b\},\{a, c\},\{b, c\},\{a, b, c\}\}$ & 6 \\
\hline F3 & $\{\{a\},\{c\},\{a, b\},\{a, c\},\{b, c\},\{a, b, c\}\}$ & 6 \\
\hline F4 & $\{\{b\},\{c\},\{a, b\},\{a, c\},\{b, c\},\{a, b, c\}\}$ & 6 \\
\hline F5 & $\{\{a\},\{a, b\},\{a, c\},\{b, c\},\{a, b, c\})$ & 5 \\
\hline F6 & $\{\{b\},\{a, b\},\{a, c\},\{b, c\},\{a, b, c\})$ & 5 \\
\hline F7 & $\{\{c\},\{a, b\},\{a, c\},\{b, c\},\{a, b, c\})$ & 5 \\
\hline F8 & $\{\{a\},\{a, b\},\{a, c\},\{a, b, c\}\}$ & 4 \\
\hline$F 9$ & $\{\{b\},\{a, b\},\{b, c\},\{a, b, c\}\}$ & 4 \\
\hline F10 & $\{\{c\},\{a, c\},\{b, c\},\{a, b, c\}\}$ & 4 \\
\hline F11 & $\{\{a, b\},\{a, c\},\{b, c\},\{a, b, c\}\}$ & 4 \\
\hline F12 & $\{\{a, b\},\{a, c\},\{a, b, c\}\}$ & 3 \\
\hline F13 & $\{\{a, b\},\{b, c\},\{a, b, c\}\}$ & 3 \\
\hline F14 & $\{\{a, c\},\{b, c\},\{a, b, c\}\}$ & 3 \\
\hline F15 & $\{\{a, b\},\{a, b, c\}\}$ & 2 \\
\hline F16 & $\{\{a, c\},\{a, b, c\}\}$ & 2 \\
\hline F17 & $\{\{b, c\},\{a, b, c\}\}$ & 2 \\
\hline F18 & $\{\{a, b, c\}\}$ & 1 \\
\hline
\end{tabular}


Lattice of topological filters

The lattice $<\Phi, \subseteq>$ is constructed as given in

over the ground set $X=\{a, b, c\}$. Note that the symbol $\subseteq$ Fig. 2 whose elements are 18 topological filters defined

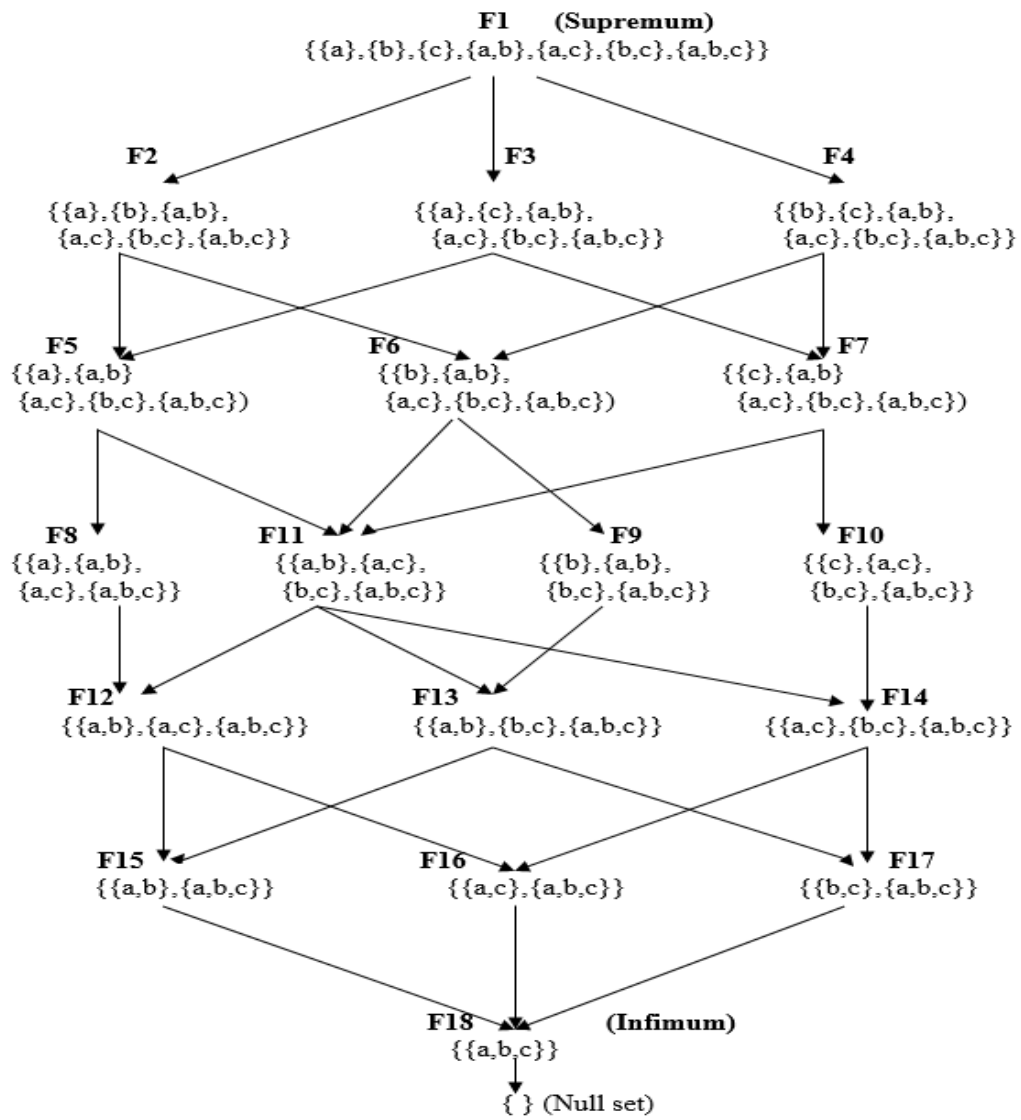

Fig. 2: Lattice diagram showing the linear filter chains over a set $X=\{a, b, c\}$

To apply STRT to a filter chain, the length of the chain should be a power of 2 . The length of the chain in this case is 7 . So the null set \{\} is considered as the eighth filter as it is a subset of any set. By applying STRT to these 48 filter chains, we get their corresponding spectra. Table 2 gives the STRT spectra of all 48 linear filter chains.

Table 2: STRT spectra of all 48 linear filter chains

Filter chain \# 1 and its STRT spectrum

\begin{tabular}{|l|l|l|l|l|}
\hline F1 & $\begin{array}{l}\{\{a\},\{b\},\{c\},\{a, b\},\{a,\} \\
\{b, c\},\{a, b, c\}\}\end{array}$ & $\begin{array}{l}\{\{a\},\{b\},\{c\},\{a, b\},\{a,\} \\
\{b, c\},\{a, b, c\}\}\end{array}$ & $\begin{array}{l}\{\{a\},\{b\},\{c\},\{a, b\},\{a, c\}, \\
\{b, c\},\{a, b, c\}\}\end{array}$ & $\begin{array}{l}\{a a\},\{b\},\{c\},\{a, b\},\{a, c\}, \\
\{b, c\},\{a, b, c\}\}\end{array}$ \\
\hline$F 2$ & $\begin{array}{l}\{\{a\},\{b\},\{a, b\},\{a, c\}, \\
\{b, c\},\{a, b, c\}\}\end{array}$ & $\begin{array}{l}\{\{a\},\{b\},\{a, b\},\{a, c\}, \\
\{b, c\},\{a, b, c\}\}\end{array}$ & $\begin{array}{l}\{\{a\},\{b\},\{a, b\},\{a, c\}, \\
\{b, c\},\{a, b, c\}\}\end{array}$ & $\{\{c\}\}$ \\
\hline$F 5$ & $\begin{array}{l}\{\{a\},\{a, b\},\{a, c\},\{b, c\}, \\
\{a, b, c\}\}\end{array}$ & $\begin{array}{l}\{\{a\},\{a, b\},\{a, c\},\{b, c\}, \\
\{a, b, c\}\}\end{array}$ & $\{\{b\},\{c\}\}$ & $\{\{b\},\{c\},\{b, c\}\}$ \\
\hline$F 8$ & $\begin{array}{l}\{\{a\},\{a, b\},\{a, c\},\{a, b, c \\
\}\end{array}$ & $\begin{array}{l}\{\{a\},\{a, b\},\{a, c\},\{a, b, c \\
\}\end{array}$ & $\{\{b\},\{b, c\}\}$ & $\{\{c\},\{b, c\}\}$ \\
\hline$F 12$ & $\{\{a, b\},\{a, c\},\{a, b, c\}\}$ & $\{\{a\},\{b\},\{c\},\{b, c\}\}$ & $\begin{array}{l}\{\{a\},\{b\},\{c\},\{a, b\},\{a, c\},\{ \\
b, c\}\}\end{array}$ & $\begin{array}{l}\{a b\},\{b\},\{c\},\{a, b\},\{a, c\}, \\
\{b, c\},\{a, b, c\}\}\end{array}$ \\
\hline F15 & $\{\{a, b\},\{a, b, c\}\}$ & $\{\{a\},\{b\},\{a, c\},\{b, c\}\}$ & $\begin{array}{l}\{\{a\},\{b\},\{a, b\},\{a, c\}, \\
\{b, c\},\{a, b, c\}\}\end{array}$ & $\{\{c\},\{a, b, c\}\}$ \\
\hline$F 18$ & $\{\{a, b, c\}\}$ & $\{\{a\},\{a, b\},\{a, c\},\{b, c\}\}$ & $\{\{b\},\{c\},\{a, b\},\{a, c\}\}$ & $\begin{array}{l}\{\{b\},\{c\},\{a, b\},\{a, c\}, \\
\{b, c\},\{a, b, c\}\}\end{array}$ \\
\hline- & $\{\Phi\}$ & $\begin{array}{l}\{\{a\},\{a, b\},\{a, c\},\{a, b, c \\
\}\}\end{array}$ & $\{\{b\},\{a, b\},\{b, c\},\{a, b, c\}\}$ & $\{\{c\},\{a, c\},\{b, c\},\{a, b, c\}\}$ \\
\hline
\end{tabular}


Filter chain \# 2 and its STRT spectrum

\begin{tabular}{|c|c|c|c|c|}
\hline $\mathrm{F} 1$ & $\begin{array}{l}\{\{a\},\{b\},\{c\},\{a, b\},\{a \\
c\},\{b, c\},\{a, b, c\}\}\end{array}$ & $\begin{array}{l}\{\{a\},\{b\},\{c\},\{a, b\},\{a, c\},\{b \\
, c\},\{a, b, c\}\}\end{array}$ & $\begin{array}{l}\{\{a\},\{b\},\{c\},\{a, b\},\{a, \\
c\}, \\
\{b, c\},\{a, b, c\}\}\end{array}$ & $\begin{array}{l}\{\{a\},\{b\},\{c\},\{a, b\},\{a, c\},\{b \\
, c\},\{a, b, c\}\}\end{array}$ \\
\hline F2 & $\begin{array}{l}\{\{a\},\{b\},\{a, b\},\{a, c\} \\
\{b, c\},\{a, b, c\}\}\end{array}$ & $\begin{array}{l}\{\{a\},\{b\},\{a, b\},\{a, c\},\{b, c\} \\
\{a, b, c\}\}\end{array}$ & $\begin{array}{l}\{\{a\},\{b\},\{a, b\},\{a, c\},\{ \\
b, c\}, \\
\{a, b, c\}\}\end{array}$ & $\{\{c\}\}$ \\
\hline F5 & $\begin{array}{l}\{a a\},\{a, b\},\{a, c\},\{b, c\}, \\
\{a, b, c\}\}\end{array}$ & $\begin{array}{l}\{\{a\},\{a, b\},\{a, c\},\{b, c\}, \\
\{a, b, c\}\}\end{array}$ & $\{\{b\},\{c\}\}$ & $\{\{b\},\{c\},\{b, c\}\}$ \\
\hline F8 & $\begin{array}{l}\{\{a\},\{a, b\},\{a, c\},\{a, b, c \\
\}\}\end{array}$ & $\{\{a\},\{a, b\},\{a, c\},\{a, b, c\}\}$ & $\{\{b\},\{b, c\}\}$ & $\{\{c\},\{b, c\}\}$ \\
\hline F12 & $\{\{a, b\},\{a, c\},\{a, b, c\}\}$ & $\{\{a\},\{b\},\{c\},\{b, c\}\}$ & $\begin{array}{l}\{\{a\},\{b\},\{c\},\{a, b\},\{a, \\
c\},\{b, c\}\}\end{array}$ & $\begin{array}{l}\{\{a\},\{b\},\{c\},\{a, b\},\{a, c\} \\
\{b, c\},\{a, b, c\}\}\end{array}$ \\
\hline F16 & $\{\{a, c\},\{a, b, c\}\}$ & $\{\{a\},\{b\},\{a, b\},\{b, c\}\}$ & $\begin{array}{l}\{\{a\},\{b\},\{a, b\},\{a, c\},\{ \\
b, c\}, \\
\{a, b, c\}\}\end{array}$ & $\{\{c\},\{a, b, c\}\}$ \\
\hline F18 & $\{\{a, b, c\}\}$ & $\{\{a\},\{a, b\},\{a, c\},\{b, c\}\}$ & $\{\{b\},\{c\},\{a, b\},\{a, c\}\}$ & $\begin{array}{l}\{\{b\},\{c\},\{a, b\},\{a, c\}, \\
\{b, c\},\{a, b, c\}\}\end{array}$ \\
\hline - & $\{\Phi\}$ & $\{\{a\},\{a, b\},\{a, c\},\{a, b, c\}\}$ & $\begin{array}{l}\{\{b\},\{a, c\},\{b, c\},\{a, b, \\
c\}\}\end{array}$ & $\{\{c\},\{a, b\},\{b, c\},\{a, b, c\}\}$ \\
\hline
\end{tabular}

Filter chain \# 3 and its STRT spectrum

\begin{tabular}{|c|c|c|c|c|}
\hline F1 & $\begin{array}{l}\{\{a\},\{b\},\{c\},\{a, b\},\{a, c\}, \\
\{b, c\},\{a, b, c\}\}\end{array}$ & $\begin{array}{l}\{\{a\},\{b\},\{c\},\{a, b\},\{a, c\}, \\
\{b, c\},\{a, b, c\}\}\end{array}$ & $\begin{array}{l}\{\{a\},\{b\},\{c\},\{a, b\},\{a, c\}, \\
\{b, c\},\{a, b, c\}\}\end{array}$ & $\begin{array}{l}\{\{a\},\{b\},\{c\},\{a, b\},\{a, c\}, \\
\{b, c\},\{a, b, c\}\}\end{array}$ \\
\hline F2 & $\begin{array}{l}\{\{a\},\{b\},\{a, b\},\{a, c\} \\
\{b, c\},\{a, b, c\}\}\end{array}$ & $\begin{array}{l}\{\{a\},\{b\},\{a, b\},\{a, c\} \\
\{b, c\},\{a, b, c\}\}\end{array}$ & $\begin{array}{l}\{a \mathrm{a}\},\{b\},\{a, b\},\{a, c\},\{b, c\}, \\
\{a, b, c\}\}\end{array}$ & $\{\{c\}\}$ \\
\hline F5 & $\begin{array}{l}\{a \mathrm{a}\},\{a, b\},\{a, c\},\{b, c\}, \\
\{a, b, c\}\}\end{array}$ & $\begin{array}{l}\{\{a\},\{a, b\},\{a, c\},\{b, c\}, \\
\{a, b, c\}\}\end{array}$ & $\{\{b\},\{c\}\}$ & $\{\{a\},\{b\},\{c\}\}$ \\
\hline F11 & $\begin{array}{l}\{a \mathrm{a}, \mathrm{b}\},\{\mathrm{a}, \mathrm{c}\},\{\mathrm{b}, \mathrm{c}\} \\
\{\mathrm{a}, \mathrm{b}, \mathrm{c}\}\}\end{array}$ & $\begin{array}{l}\{\{a, b\},\{a, c\},\{b, c\},\{a, b, c\} \\
\}\end{array}$ & $\{\{a\},\{b\}\}$ & $\{\{a\},\{c\}\}$ \\
\hline F12 & $\{\{a, b\},\{a, c\},\{a, b, c\}\}$ & $\{\{a\},\{b\},\{c\},\{b, c\}\}$ & $\begin{array}{l}\{\{a\},\{b\},\{c\},\{a, b\},\{a, c\} \\
\{b, c\}\}\end{array}$ & $\begin{array}{l}\{\{a\},\{b\},\{c\},\{a, b\},\{a, c\}, \\
\{b, c\},\{a, b, c\}\}\end{array}$ \\
\hline F15 & $\{\{a, b\},\{a, b, c\}\}$ & $\{\{a\},\{b\},\{a, c\},\{b, c\}\}$ & $\begin{array}{l}\{\{a\},\{b\},\{a, c\},\{a, b\},\{b, c\}, \\
\{a, b, c\}\}\end{array}$ & $\{\{c\},\{a, b, c\}\}$ \\
\hline F18 & $\{\{a, b, c\}\}$ & $\{\{a\},\{a, b\},\{a, c\},\{b, c\}\}$ & $\{\{b\},\{c\},\{a, b\},\{a, c\}\}$ & $\begin{array}{l}\{\{a\},\{b\},\{c\},\{a, b\},\{a, c\}, \\
\{a, b, c\}\}\end{array}$ \\
\hline - & $\{\Phi\}$ & $\begin{array}{l}\{\{a, b\},\{a, c\},\{b, c\},\{a, b, c\} \\
\}\end{array}$ & $\{\{a\},\{b\},\{a, b\},\{a, b, c\}\}$ & $\{\{a\},\{c\},\{a, c\},\{a, b, c\}\}$ \\
\hline
\end{tabular}

Filter chain \# 4 and its STRT spectrum

\begin{tabular}{|c|c|c|c|c|}
\hline $\mathrm{F} 1$ & $\begin{array}{l}\{\{a\},\{b\},\{c\},\{a, b\},\{a, \\
c\}, \\
\{b, c\},\{a, b, c\}\}\end{array}$ & $\begin{array}{l}\{\{a\},\{b\},\{c\},\{a, b\},\{a, \\
c\}, \\
\{b, c\},\{a, b, c\}\}\end{array}$ & $\begin{array}{l}\{\{a\},\{b\},\{c\},\{a, b\},\{a, c\} \\
\{b, c\},\{a, b, c\}\}\end{array}$ & $\begin{array}{l}\{\{a\},\{b\},\{c\},\{a, b\},\{a, c\} \\
\{b, c\},\{a, b, c\}\}\end{array}$ \\
\hline F2 & $\begin{array}{l}\{\{a\},\{b\},\{a, b\},\{a, c\} \\
\{b, c\},\{a, b, c\}\}\end{array}$ & $\begin{array}{l}\{\{a\},\{b\},\{a, b\},\{a, c\} \\
\{b, c\},\{a, b, c\}\}\end{array}$ & $\begin{array}{l}\{\{a\},\{b\},\{a, b\},\{a, c\},\{b, c\}, \\
\{a, b, c\}\}\end{array}$ & $\{\{c\}\}$ \\
\hline F5 & $\begin{array}{l}\{\{a\},\{a, b\},\{a, c\},\{b, c\} \\
\{a, b, c\}\}\end{array}$ & $\begin{array}{l}\{\{a\},\{a, b\},\{a, c\},\{b, c\} \\
\{a, b, c\}\}\end{array}$ & $\{\{b\},\{c\}\}$ & $\{\{a\},\{b\},\{c\}\}$ \\
\hline $\mathrm{F} 11$ & $\begin{array}{l}\{\{a, b\},\{a, c\},\{b, c\} \\
\{a, b, c\}\}\end{array}$ & $\begin{array}{l}\{\{a, b\},\{a, c\},\{b, c\} \\
\{a, b, c\}\}\end{array}$ & $\{\{a\},\{b\}\}$ & $\{\{a\},\{c\}\}$ \\
\hline F12 & $\{\{a, b\},\{a, c\},\{a, b, c\}\}$ & $\{\{a\},\{b\},\{c\},\{b, c\}\}$ & $\begin{array}{l}\{\{a\},\{b\},\{c\},\{a, b\},\{a, c\},\{b, \\
c\}\}\end{array}$ & $\begin{array}{l}\{\{a\},\{b\},\{c\},\{a, b\},\{a, c\}, \\
\{b, c\},\{a, b, c\}\}\end{array}$ \\
\hline F16 & $\{\{a, c\},\{a, b, c\}\}$ & $\{\{a\},\{b\},\{a, b\},\{b, c\}\}$ & $\begin{array}{l}\{\{a\},\{b\},\{a, b\},\{a, c\} \\
\{b, c\},\{a, b, c\}\}\end{array}$ & $\{\{c\},\{a, b, c\}\}$ \\
\hline F18 & $\{\{a, b, c\}\}$ & $\begin{array}{l}\{\{a\},\{a, b\},\{a, c\},\{b, c\} \\
\}\end{array}$ & $\{\{b\},\{c\},\{a, b\},\{a, c\}\}$ & $\begin{array}{l}\{\{a\},\{b\},\{c\},\{a, b\},\{a, c\}, \\
\{a, b, c\}\}\end{array}$ \\
\hline- & $\{\Phi\}$ & $\begin{array}{l}\{\{a, b\},\{a, c\},\{b, c\} \\
\{a, b, c\}\}\end{array}$ & $\{\{a\},\{b\},\{a, c\},\{a, b, c\}\}$ & $\{\{a\},\{c\},\{a, b\},\{a, b, c\}\}$ \\
\hline
\end{tabular}


Filter chain \# 5 and its STRT spectrum

\begin{tabular}{|c|c|c|c|c|}
\hline F1 & $\begin{array}{l}\{\{a\},\{b\},\{c\},\{a, b\},\{a, c\}, \\
\{b, c\},\{a, b, c\}\}\end{array}$ & $\begin{array}{l}\{\{a\},\{b\},\{c\},\{a, b\},\{a, c\}, \\
\{b, c\},\{a, b, c\}\}\end{array}$ & $\begin{array}{l}\{\{a\},\{b\},\{c\},\{a, b\},\{a, c\}, \\
\{b, c\},\{a, b, c\}\}\end{array}$ & $\begin{array}{l}\{\{a\},\{b\},\{c\},\{a, b\},\{a, c\}, \\
\{b, c\},\{a, b, c\}\}\end{array}$ \\
\hline F2 & $\begin{array}{l}\{\{a\},\{b\},\{a, b\},\{a, c\} \\
\{b, c\},\{a, b, c\}\}\end{array}$ & $\begin{array}{l}\{\{a\},\{b\},\{a, b\},\{a, c\} \\
\{b, c\},\{a, b, c\}\}\end{array}$ & $\begin{array}{l}\{a \mathrm{a}\},\{b\},\{a, b\},\{a, c\} \\
\{b, c\},\{a, b, c\}\}\end{array}$ & $\{\{c\}\}$ \\
\hline F5 & $\begin{array}{l}\{\{a\},\{a, b\},\{a, c\},\{b, c\}, \\
\{a, b, c\}\}\end{array}$ & $\begin{array}{l}\{\{a\},\{a, b\},\{a, c\},\{b, c\}, \\
\{a, b, c\}\}\end{array}$ & $\{\{b\},\{c\}\}$ & $\{\{a\},\{b\},\{c\}\}$ \\
\hline F11 & $\begin{array}{l}\{\{a, b\},\{a, c\},\{b, c\} \\
\{a, b, c\}\}\end{array}$ & $\begin{array}{l}\{\{a, b\},\{a, c\},\{b, c\} \\
\{a, b, c\}\}\end{array}$ & $\{\{a\},\{b\}\}$ & $\{\{a\},\{c\}\}$ \\
\hline F13 & $\{\{a, b\},\{b, c\},\{a, b, c\}\}$ & $\{\{a\},\{b\},\{c\},\{a, c\}\}$ & $\begin{array}{l}\{\{a\},\{b\},\{c\},\{a, b\} \\
\{a, c\},\{b, c\}\}\end{array}$ & $\begin{array}{l}\{\{a\},\{b\},\{c\},\{a, b\},\{a, c\}, \\
\{b, c\},\{a, b, c\}\}\end{array}$ \\
\hline F15 & $\{\{a, b\},\{a, b, c\}\}$ & $\{\{a\},\{b\},\{a, c\},\{b, c\}\}$ & $\begin{array}{l}\{\{a\},\{b\},\{a, b\},\{a, c\}, \\
\{b, c\},\{a, b, c\}\}\end{array}$ & $\{\{c\},\{a, b, c\}\}$ \\
\hline F18 & $\{\{a, b, c\}\}$ & $\{\{a\},\{a, b\},\{a, c\},\{b, c\}\}$ & $\{\{b\},\{c\},\{a, b\},\{b, c\}\}$ & $\begin{array}{l}\{\{a\},\{b\},\{c\},\{a, b\},\{b, c\}, \\
\{a, b, c\}\}\end{array}$ \\
\hline- & $\{\Phi\}$ & $\{a \mathrm{a}, \mathrm{b}\},\{\mathrm{a}, \mathrm{c}\},\{\mathrm{b}, \mathrm{c}\},\{\mathrm{a}, \mathrm{b}, \mathrm{c}\}\}$ & $\{\{a\},\{b\},\{a, b\},\{a, b, c\}\}$ & $\{\{a\},\{c\},\{b, c\},\{a, b, c\}\}$ \\
\hline
\end{tabular}

Filter chain \# 6 and its STRT spectrum

\begin{tabular}{|c|c|c|c|c|}
\hline F1 & $\begin{array}{l}\{\{a\},\{b\},\{c\},\{a, b\},\{ \\
a, c\}, \\
\{b, c\},\{a, b, c\}\}\end{array}$ & $\begin{array}{l}\{\{a\},\{b\},\{c\},\{a, b\},\{a, c\}, \\
\{b, c\},\{a, b, c\}\}\end{array}$ & $\begin{array}{l}\{\{a\},\{b\},\{c\},\{a, b\},\{a, c \\
\}, \\
\{b, c\},\{a, b, c\}\}\end{array}$ & $\begin{array}{l}\{\{a\},\{b\},\{c\},\{a, b\},\{a, c\}, \\
\{b, c\},\{a, b, c\}\}\end{array}$ \\
\hline F2 & $\begin{array}{l}\{\{a\},\{b\},\{a, b\},\{a, c\}, \\
\{b, c\},\{a, b, c\}\}\end{array}$ & $\begin{array}{l}\{\{a\},\{b\},\{a, b\},\{a, c\}, \\
\{b, c\},\{a, b, c\}\}\end{array}$ & $\begin{array}{l}\{\{a\},\{b\},\{a, b\},\{a, c\} \\
\{b, c\},\{a, b, c\}\}\end{array}$ & $\{\{c\}\}$ \\
\hline F5 & $\begin{array}{l}\{\{a\},\{a, b\},\{a, c\},\{b, c \\
\}, \\
\{a, b, c\}\}\end{array}$ & $\begin{array}{l}\{\{a\},\{a, b\},\{a, c\},\{b, c\}, \\
\{a, b, c\}\}\end{array}$ & $\{\{b\},\{c\}\}$ & $\{\{a\},\{b\},\{c\}\}$ \\
\hline F11 & $\begin{array}{l}\{\{a, b\},\{a, c\},\{b, c\},\{a \\
, b, c\}\}\end{array}$ & $\begin{array}{l}\{\{a, b\},\{a, c\},\{b, c\},\{a, b, c \\
\}\}\end{array}$ & $\{\{a\},\{b\}\}$ & $\{\{a\},\{c\}\}$ \\
\hline F13 & $\{\{a, b\},\{b, c\},\{a, b, c\}\}$ & $\{\{a\},\{b\},\{c\},\{a, c\}\}$ & $\begin{array}{l}\{\{a\},\{b\},\{c\},\{a, b\},\{a, c \\
\}, \\
\{b, c\}\}\end{array}$ & $\begin{array}{l}\{\{a\},\{b\},\{c\},\{a, b\},\{a, c\}, \\
\{b, c\},\{a, b, c\}\}\end{array}$ \\
\hline F17 & $\{\{b, c\},\{a, b, c\}\}$ & $\{\{a\},\{b\},\{a, b\},\{a, c\}\}$ & $\begin{array}{l}\{\{a\},\{b\},\{a, b\},\{a, c\},\{b \\
, c\}, \\
\{a, b, c\}\}\end{array}$ & $\{\{c\},\{a, b, c\}\}$ \\
\hline F18 & $\{\{a, b, c\}\}$ & $\{\{a\},\{a, b\},\{a, c\},\{b, c\}\}$ & $\{\{b\},\{c\},\{a, b\},\{b, c\}\}$ & $\begin{array}{l}\{\{a\},\{b\},\{c\},\{a, b\},\{b, c\}, \\
\{a, b, c\}\}\end{array}$ \\
\hline- & $\{\Phi\}$ & $\begin{array}{l}\{\{a, b\},\{a, c\},\{b, c\},\{a, b, c \\
\}\}\end{array}$ & $\{\{a\},\{b\},\{b, c\},\{a, b, c\}\}$ & $\{\{a\},\{c\},\{a, b\},\{a, b, c\}\}$ \\
\hline
\end{tabular}

Filter chain \# 7 and its STRT spectrum

\begin{tabular}{|l|l|l|l|l|}
\hline F1 & $\begin{array}{l}\{\{a\},\{b\},\{c\},\{a, b \\
\},\{a, c\},\{b, c\},\{a, b, c\}\}\end{array}$ & $\begin{array}{l}\{\{a\},\{b\},\{c\},\{a, b\},\{a, c\}, \\
\{b, c\},\{a, b, c\}\}\end{array}$ & $\begin{array}{l}\{\{a\},\{b\},\{c\},\{a, b\},\{a, c \\
\{b, c\},\{a, b, c\}\}\end{array}$ & $\begin{array}{l}\{\{a\},\{b\},\{c\},\{a, b\},\{a, c\}, \\
\{b, c\},\{a, b, c\}\}\end{array}$ \\
\hline F2 & $\begin{array}{l}\{\{a\},\{b\},\{a, b\},\{a, c\}, \\
\{b, c\},\{a, b, c\}\}\end{array}$ & $\begin{array}{l}\{\{a\},\{b\},\{a, b\},\{a, c\}, \\
\{b, c\},\{a, b, c\}\}\end{array}$ & $\begin{array}{l}\{\{a\},\{b\},\{a, b\},\{a, c\}, \\
\{b, c\},\{a, b, c\}\}\end{array}$ & $\{\{c\}\}$ \\
\hline F5 & $\begin{array}{l}\{\{a\},\{a, b\},\{a, c\},\{b, c\}, \\
\{a, b, c\}\}\end{array}$ & $\begin{array}{l}\{\{a\},\{a, b\},\{a, c\},\{b, c\}, \\
\{a, b, c\}\}\end{array}$ & $\{\{b\},\{c\}\}$ & $\{\{a\},\{b\},\{c\}\}$ \\
\hline$F 11$ & $\begin{array}{l}\{\{a, b\},\{a, c\},\{b, c\},\{a, b, c \\
\}\}\end{array}$ & $\begin{array}{l}\{\{a, b\},\{a, c\},\{b, c\},\{a, b, c \\
\}\}\end{array}$ & $\{\{a\},\{b\}\}$ & $\{\{a\},\{c\}\}$ \\
\hline F14 & $\{\{a, c\},\{b, c\},\{a, b, c\}\}$ & $\{\{a\},\{b\},\{c\},\{a, b\}\}$ & $\begin{array}{l}\{\{a\},\{b\},\{c\},\{a, b\},\{a, c \\
\},\{b, c\}\}\end{array}$ & $\begin{array}{l}\{\{a\},\{b\},\{c\},\{a, b\},\{a, c\}, \\
\{b, c\},\{a, b, c\}\}\end{array}$ \\
\hline F16 & $\{\{a, c\},\{a, b, c\}\}$ & $\{\{a\},\{b\},\{a, b\},\{b, c\}\}$ & $\begin{array}{l}\{\{a\},\{b\},\{a, b\},\{a, c\},\{b, \\
c\}, \\
\{a, b, c\}\}\end{array}$ & $\{\{c\},\{a, b, c\}\}$ \\
\hline$F 18$ & $\{\{a, b, c\}\}$ & $\{\{a\},\{a, b\},\{a, c\},\{b, c\}\}$ & $\{\{b\},\{c\},\{a, c\},\{b, c\}\}$ & $\begin{array}{l}\{\{a\},\{b\},\{c\},\{a, c\},\{b, c\}, \\
\{a, b, c\}\}\end{array}$ \\
\hline- & $\{\Phi\}$ & $\begin{array}{l}\{\{a, b\},\{a, c\},\{b, c\},\{a, b, c \\
\}\}\end{array}$ & $\{\{a\},\{b\},\{a, c\},\{a, b, c\}\}$ & $\{\{a\},\{c\},\{b, c\},\{a, b, c\}\}$ \\
\hline
\end{tabular}


Filter chain \# 8 and its STRT spectrum

\begin{tabular}{|c|c|c|c|c|}
\hline $\mathrm{F} 1$ & $\begin{array}{l}\{\{a\},\{b\},\{c\},\{a, b\},\{a, c\}, \\
\{b, c\},\{a, b, c\}\}\end{array}$ & $\begin{array}{l}\{\{a\},\{b\},\{c\},\{a, b\},\{a, c\}, \\
\{b, c\},\{a, b, c\}\}\end{array}$ & $\begin{array}{l}\{\{a\},\{b\},\{c\},\{a, b\},\{a, c\}, \\
\{b, c\},\{a, b, c\}\}\end{array}$ & $\begin{array}{l}\{\{a\},\{b\},\{c\},\{a, b\},\{a \\
, c\}, \\
\{b, c\},\{a, b, c\}\}\end{array}$ \\
\hline F2 & $\begin{array}{l}\{\{a\},\{b\},\{a, b\},\{a, c\} \\
\{b, c\},\{a, b, c\}\}\end{array}$ & $\begin{array}{l}\{\{a\},\{b\},\{a, b\},\{a, c\} \\
\{b, c\},\{a, b, c\}\}\end{array}$ & $\begin{array}{l}\{\{a\},\{b\},\{a, b\},\{a, c\} \\
\{b, c\},\{a, b, c\}\}\end{array}$ & $\{\{c\}\}$ \\
\hline F5 & $\begin{array}{l}\{\{a\},\{a, b\},\{a, c\} \\
\{b, c\},\{a, b, c\}\}\end{array}$ & $\begin{array}{l}\{\{a\},\{a, b\},\{a, c\},\{b, c\} \\
\{a, b, c\}\}\end{array}$ & $\{\{\mathrm{b}\},\{\mathrm{c}\}\}$ & $\{\{a\},\{b\},\{c\}\}$ \\
\hline $\begin{array}{l}\mathrm{F} 1 \\
1\end{array}$ & $\begin{array}{l}\{\{a, b\},\{a, c\},\{b, c\},\{a, b, c \\
\}\}\end{array}$ & $\begin{array}{l}\{\{a, b\},\{a, c\},\{b, c\},\{a, b, c \\
\}\}\end{array}$ & $\{\{a\},\{b\}\}$ & $\{\{a\},\{c\}\}$ \\
\hline $\begin{array}{l}\mathrm{F} 1 \\
4\end{array}$ & $\{\{a, c\},\{b, c\},\{a, b, c\}\}$ & $\{\{a\},\{b\},\{c\},\{a, b\}\}$ & $\begin{array}{l}\{\{a\},\{b\},\{c\},\{a, b\},\{a, c\},\{b, c \\
\}\}\end{array}$ & $\begin{array}{l}\{\{a\},\{b\},\{c\},\{a, b\},\{a \\
, c\}, \\
\{b, c\},\{a, b, c\}\}\end{array}$ \\
\hline $\begin{array}{l}\mathrm{F} 1 \\
7\end{array}$ & $\{\{b, c\},\{a, b, c\}\}$ & $\{\{a\},\{b\},\{a, b\},\{a, c\}\}$ & $\begin{array}{l}\{\{a\},\{b\},\{a, b\},\{a, c\},\{b, c\}, \\
\{a, b, c\}\}\end{array}$ & $\{\{c\},\{a, b, c\}\}$ \\
\hline $\begin{array}{l}\mathrm{F} 1 \\
8\end{array}$ & $\{\{a, b, c\}\}$ & $\{\{a\},\{a, b\},\{a, c\},\{b, c\}\}$ & $\{\{b\},\{c\},\{a, c\},\{b, c\}\}$ & $\begin{array}{l}\{\{a\},\{b\},\{c\},\{a, c\},\{b \\
, c\}, \\
\{a, b, c\}\}\end{array}$ \\
\hline - & $\{\Phi\}$ & $\begin{array}{l}\{\{a, b\},\{a, c\},\{b, c\},\{a, b, c \\
\}\}\end{array}$ & $\{\{a\},\{b\},\{b, c\},\{a, b, c\}\}$ & $\{\{a\},\{c\},\{a, c\},\{a, b, c$ \\
\hline
\end{tabular}

Filter chain \# 9 and its STRT spectrum

\begin{tabular}{|c|c|c|c|c|}
\hline F1 & $\begin{array}{l}\{\{a\},\{b\},\{c\},\{a, b\},\{a, c\} \\
\{b, c\},\{a, b, c\}\}\end{array}$ & $\begin{array}{l}\{\{a\},\{b\},\{c\},\{a, b\},\{a, c\} \\
\{b, c\},\{a, b, c\}\}\end{array}$ & $\begin{array}{l}\{\{a\},\{b\},\{c\},\{a, b\},\{a, c\} \\
\{b, c\},\{a, b, c\}\}\end{array}$ & $\begin{array}{l}\{\{a\},\{b\},\{c\},\{a, b\},\{a \\
, c\}, \\
\{b, c\},\{a, b, c\}\}\end{array}$ \\
\hline F2 & $\begin{array}{l}\{\{a\},\{b\},\{a, b\},\{a, c\} \\
\{b, c\},\{a, b, c\}\}\end{array}$ & $\begin{array}{l}\{\{a\},\{b\},\{a, b\},\{a, c\} \\
\{b, c\},\{a, b, c\}\}\end{array}$ & $\begin{array}{l}\{\{a\},\{b\},\{a, b\},\{a, c\}, \\
\{b, c\},\{a, b, c\}\}\end{array}$ & $\{\{c\}\}$ \\
\hline F6 & $\begin{array}{l}\{\{b\},\{a, b\},\{a, c\},\{b, c\},\{a, \\
b, c\}\}\end{array}$ & $\begin{array}{l}\{\{b\},\{a, b\},\{a, c\},\{b, c\},\{a, \\
b, c\}\}\end{array}$ & $\{\{a\},\{c\}\}$ & $\{\{a\},\{b\},\{c\}\}$ \\
\hline $\mathrm{F} 11$ & $\begin{array}{l}\{\{a, b\},\{a, c\},\{b, c\},\{a, b, c\} \\
\}\end{array}$ & $\begin{array}{l}\{\{a, b\},\{a, c\},\{b, c\},\{a, b, c\} \\
\}\end{array}$ & $\{\{a\},\{b\}\}$ & $\{\{b\},\{c\}\}$ \\
\hline $\mathrm{F} 12$ & $\{\{a, b\},\{a, c\},\{a, b, c\}\}$ & $\{\{a\},\{b\},\{c\},\{b, c\}\}$ & $\begin{array}{l}\{\{a\},\{b\},\{c\},\{a, b\},\{a, c\},\{b \\
, c\}\}\end{array}$ & $\begin{array}{l}\{\{a\},\{b\},\{c\},\{a, b\},\{a \\
, c\}, \\
\{b, c\},\{a, b, c\}\}\end{array}$ \\
\hline $\mathrm{F} 15$ & $\{\{a, b\},\{a, b, c\}\}$ & $\{\{a\},\{b\},\{a, c\},\{b, c\}\}$ & $\begin{array}{l}\{\{a\},\{b\},\{a, b\},\{a, c\},\{b, c\}, \\
\{a, b, c\}\}\end{array}$ & $\{\{c\},\{a, b, c\}\}$ \\
\hline F18 & $\{\{a, b, c\}\}$ & $\{\{b\},\{a, b\},\{a, c\},\{b, c\}\}$ & $\{\{a\},\{c\},\{a, b\},\{a, c\}\}$ & $\begin{array}{l}\{\{a\},\{b\},\{c\},\{a, b\} \\
\{a, c\},\{a, b, c\}\}\end{array}$ \\
\hline & $\{\Phi\}$ & $\{\{a, b\},\{a, c\},\{b, c\},\{a, b, c\}$ & $\{\{a\},\{b\},\{a, b\},\{a, b, c\}\}$ & $\begin{array}{l}\{\{b\},\{c\},\{a, c\},\{a, b, c \\
\}\}\end{array}$ \\
\hline
\end{tabular}

Filter chain \# 10 and its STRT spectrum

\begin{tabular}{|l|l|l|l|l|}
\hline F1 & $\begin{array}{l}\{\{a\},\{b\},\{c\},\{a, b\},\{a, c\}, \\
\{b, c\},\{a, b, c\}\}\end{array}$ & $\begin{array}{l}\{\{a\},\{b\},\{c\},\{a, b\},\{a, c\} \\
,\{b, c\},\{a, b, c\}\}\end{array}$ & $\begin{array}{l}\{\{a\},\{b\},\{c\},\{a, b\},\{a, c\}, \\
\{b, c\},\{a, b, c\}\}\end{array}$ & $\begin{array}{l}\{\{a\},\{b\},\{c\},\{a, b\},\{a, c \\
\},\{b, c\},\{a, b, c\}\}\end{array}$ \\
\hline F2 & $\begin{array}{l}\{\{a\},\{b\},\{a, b\},\{a, c\},\{b, c\} \\
\{a, b, c\}\}\end{array}$ & $\begin{array}{l}\{\{a\},\{b\},\{a, b\},\{a, c\}, \\
\{b, c\},\{a, b, c\}\}\end{array}$ & $\begin{array}{l}\{\{a\},\{b\},\{a, b\},\{a, c\}, \\
\{b, c\},\{a, b, c\}\}\end{array}$ & $\{\{c\}\}$ \\
\hline F6 & $\begin{array}{l}\{\{b\},\{a, b\},\{a, c\},\{b, c\},\{a, \\
b, c\}\}\end{array}$ & $\begin{array}{l}\{\{b\},\{a, b\},\{a, c\},\{b, c\}, \\
\{a, b, c\}\}\end{array}$ & $\{\{a\},\{c\}\}$ & $\{\{a\},\{b\},\{c\}\}$ \\
\hline$F 11$ & $\begin{array}{l}\{\{a, b\},\{a, c\},\{b, c\},\{a, b, c\} \\
\}\end{array}$ & $\begin{array}{l}\{\{a, b\},\{a, c\},\{b, c\},\{a, b, \\
c\}\}\end{array}$ & $\{\{a\},\{b\}\}$ & $\{\{b\},\{c\}\}$ \\
\hline$F 12$ & $\{\{a, b\},\{a, c\},\{a, b, c\}\}$ & $\{\{a\},\{b\},\{c\},\{b, c\}\}$ & $\begin{array}{l}\{\{a\},\{b\},\{c\},\{a, b\},\{a, c\},\{b, c \\
f\}\end{array}$ & $\begin{array}{l}\{\{a\},\{b\},\{c\},\{a, b\},\{a, c \\
\{b, c\},\{a, b, c\}\}\end{array}$ \\
\hline$F 16$ & $\{\{a, c\},\{a, b, c\}\}$ & $\{\{a\},\{b\},\{a, b\},\{b, c\}\}$ & $\begin{array}{l}\{\{a\},\{b\},\{a, b\},\{a, c\},\{b, c\}, \\
\{a, b, c\}\}\end{array}$ & $\{\{c\},\{a, b, c\}\}$ \\
\hline F18 & $\{\{a, b, c\}\}$ & $\{\{b\},\{a, b\},\{a, c\},\{b, c\}\}$ & $\{\{a\},\{c\},\{a, b\},\{a, c\}\}$ & $\begin{array}{l}\{\{a\},\{b\},\{c\},\{a, b\},\{a, c \\
\},\{a, b, c\}\}\end{array}$ \\
\hline- & $\{\Phi\}$ & $\begin{array}{l}\{\{a, b\},\{a, c\},\{b, c\},\{a, b, \\
c\}\}\end{array}$ & $\{\{a\},\{b\},\{a, c\},\{a, b, c\}\}$ & $\{\{b\},\{c\},\{a, b\},\{a, b, c\}\}$ \\
\hline
\end{tabular}


Filter chain \#11 and its STRT spectrum

\begin{tabular}{|c|c|c|c|c|}
\hline F1 & $\begin{array}{l}\{a \mathrm{a}\},\{b\},\{c\},\{a, b\},\{a, c\} \\
\{b, c\},\{a, b, c\}\}\end{array}$ & $\begin{array}{l}\{\{a\},\{b\},\{c\},\{a, b\},\{a, c \\
\} \\
\{b, c\},\{a, b, c\}\}\end{array}$ & $\begin{array}{l}\{\{a\},\{b\},\{c\},\{a, b\},\{a, c\} \\
\{b, c\},\{a, b, c\}\}\end{array}$ & $\begin{array}{l}\{\{a\},\{b\},\{c\},\{a, b\},\{a, \\
c\}, \\
\{b, c\},\{a, b, c\}\}\end{array}$ \\
\hline F2 & $\begin{array}{l}\{\{a\},\{b\},\{a, b\},\{a, c\} \\
\{b, c\},\{a, b, c\}\}\end{array}$ & $\begin{array}{l}\{a \mathrm{a}\},\{b\},\{a, b\},\{a, c\} \\
\{b, c\},\{a, b, c\}\}\end{array}$ & $\begin{array}{l}\{\{a\},\{b\},\{a, b\},\{a, c\},\{b, c\}, \\
\{a, b, c\}\}\end{array}$ & $\{\{c\}\}$ \\
\hline F6 & $\begin{array}{l}\{\{b\},\{a, b\},\{a, c\},\{b, c\},\{a \\
, b, c\}\}\end{array}$ & $\begin{array}{l}\{\{b\},\{a, b\},\{a, c\},\{b, c\}, \\
\{a, b, c\}\}\end{array}$ & $\{\{a\},\{c\}\}$ & $\{\{a\},\{b\},\{c\}\}$ \\
\hline F11 & $\begin{array}{l}\{\{a, b\},\{a, c\},\{b, c\},\{a, b, c \\
\}\}\end{array}$ & $\begin{array}{l}\{\{a, b\},\{a, c\},\{b, c\} \\
\{a, b, c\}\}\end{array}$ & $\{\{a\},\{b\}\}$ & $\{\{\mathrm{b}\},\{\mathrm{c}\}\}$ \\
\hline F13 & $\{\{a, b\},\{b, c\},\{a, b, c\}\}$ & $\{\{a\},\{b\},\{c\},\{a, c\}\}$ & $\begin{array}{l}\{\{a\},\{b\},\{c\},\{a, b\},\{a, c\},\{b, \\
c\}\}\end{array}$ & $\begin{array}{l}\{\{a\},\{b\},\{c\},\{a, b\},\{a, \\
c\}, \\
\{b, c\},\{a, b, c\}\}\end{array}$ \\
\hline F15 & $\{\{a, b\},\{a, b, c\}\}$ & $\{\{a\},\{b\},\{a, c\},\{b, c\}\}$ & $\begin{array}{l}\{\{a\},\{b\},\{a, b\},\{a, c\},\{b, c\}, \\
\{a, b, c\}\}\end{array}$ & $\{\{c\},\{a, b, c\}\}$ \\
\hline F18 & $\{\{a, b, c\}\}$ & $\{\{b\},\{a, b\},\{a, c\},\{b, c\}\}$ & $\{\{a\},\{c\},\{a, b\},\{b, c\}\}$ & $\begin{array}{l}\{\{a\},\{b\},\{c\},\{a, b\},\{b, \\
c\}, \\
\{a, b, c\}\}\end{array}$ \\
\hline & $\{\Phi\}$ & $\begin{array}{l}\{\{a, b\},\{a, c\},\{b, c\},\{a, b, \\
c\}\}\end{array}$ & $\{\{a\},\{b\},\{a, b\},\{a, b, c\}\}$ & $\begin{array}{l}\{\{b\},\{c\},\{b, c\},\{a, b, c\} \\
\}\end{array}$ \\
\hline
\end{tabular}

Filter chain \# 12 and its STRT spectrum

\begin{tabular}{|c|c|c|c|c|}
\hline F1 & $\begin{array}{l}\{\{a\},\{b\},\{c\},\{a, b\},\{a, c\}, \\
\{b, c\},\{a, b, c\}\}\end{array}$ & $\begin{array}{l}\{\{a\},\{b\},\{c\},\{a, b\},\{a, c \\
\}, \\
\{b, c\},\{a, b, c\}\}\end{array}$ & $\begin{array}{l}\{\{a\},\{b\},\{c\},\{a, b\},\{a, c\} \\
\{b, c\},\{a, b, c\}\}\end{array}$ & $\begin{array}{l}\{\{a\},\{b\},\{c\},\{a, b\},\{a, \\
c\}, \\
\{b, c\},\{a, b, c\}\}\end{array}$ \\
\hline F2 & $\begin{array}{l}\{\{a\},\{b\},\{a, b\},\{a, c\}, \\
\{b, c\},\{a, b, c\}\}\end{array}$ & $\begin{array}{l}\{\{a\},\{b\},\{a, b\},\{a, c\} \\
\{b, c\},\{a, b, c\}\}\end{array}$ & $\begin{array}{l}\{\{a\},\{b\},\{a, b\},\{a, c\},\{b, c\}, \\
\{a, b, c\}\}\end{array}$ & $\{\{c\}\}$ \\
\hline F6 & $\begin{array}{l}\left\{\begin{array}{l}\{b\},\{a, b\},\{a, c\},\{b, c\} \\
\{a, b, c\}\}\end{array}\right.\end{array}$ & $\begin{array}{l}\{\{b\},\{a, b\},\{a, c\},\{b, c\} \\
\{a, b, c\}\}\end{array}$ & $\{\{a\},\{c\}\}$ & $\{\{\mathrm{a}\},\{\mathrm{b}\},\{\mathrm{c}\}\}$ \\
\hline F11 & $\begin{array}{l}\{\{a, b\},\{a, c\},\{b, c\},\{a, b, c\} \\
\}\end{array}$ & $\begin{array}{l}\{\{a, b\},\{a, c\},\{b, c\},\{a, b, \\
c\}\}\end{array}$ & $\{\{a\},\{b\}\}$ & $\{\{\mathrm{b}\},\{\mathrm{c}\}\}$ \\
\hline F13 & $\{\{a, b\},\{b, c\},\{a, b, c\}\}$ & $\{\{a\},\{b\},\{c\},\{a, c\}\}$ & $\begin{array}{l}\{\{a\},\{b\},\{c\},\{a, b\},\{a, c\},\{b, c \\
\}\}\end{array}$ & $\begin{array}{l}\{\{a\},\{b\},\{c\},\{a, b\},\{a, \\
c\}, \\
\{b, c\},\{a, b, c\}\}\end{array}$ \\
\hline F17 & $\{\{b, c\},\{a, b, c\}\}$ & $\{\{a\},\{b\},\{a, b\},\{a, c\}\}$ & $\begin{array}{l}\{\{a\},\{b\},\{a, b\},\{a, c\},\{b, c\}, \\
\{a, b, c\}\}\end{array}$ & $\{\{c\},\{a, b, c\}\}$ \\
\hline F18 & $\{\{a, b, c\}\}$ & $\{\{b\},\{a, b\},\{a, c\},\{b, c\}\}$ & $\{\{a\},\{c\},\{a, b\},\{b, c\}\}$ & $\begin{array}{l}\{\{a\},\{b\},\{c\},\{a, b\},\{b, \\
c\}, \\
\{a, b, c\}\}\end{array}$ \\
\hline - & $\{\Phi\}$ & $\begin{array}{l}\{\{a, b\},\{a, c\},\{b, c\},\{a, b, \\
c\}\}\end{array}$ & $\{\{a\},\{b\},\{b, c\},\{a, b, c\}\}$ & $\begin{array}{l}\{\{b\},\{c\},\{a, b\},\{a, b, c\} \\
\}\end{array}$ \\
\hline
\end{tabular}

Filter chain \# 13 and its STRT spectrum

\begin{tabular}{|c|c|c|c|c|}
\hline $\mathrm{F} 1$ & $\begin{array}{c}\{\{a\},\{b\},\{c\},\{a, b\},\{a, c\} \\
\{b, c\},\{a, b, c\}\}\end{array}$ & $\begin{array}{c}\{\{a\},\{b\},\{c\},\{a, b\},\{a, c \\
\}, \\
\{b, c\},\{a, b, c\}\}\end{array}$ & $\begin{array}{c}\{\{a\},\{b\},\{c\},\{a, b\},\{a, c \\
\}, \\
\{b, c\},\{a, b, c\}\}\end{array}$ & $\begin{array}{c}\{\{a\},\{b\},\{c\},\{a, b\},\{a, c\}, \\
\{b, c\},\{a, b, c\}\}\end{array}$ \\
\hline F2 & $\begin{array}{c}\{\{a\},\{b\},\{a, b\},\{a, c\} \\
\{b, c\},\{a, b, c\}\}\end{array}$ & $\begin{array}{c}\{\{a\},\{b\},\{a, b\},\{a, c\} \\
\quad\{b, c\},\{a, b, c\}\}\end{array}$ & $\begin{array}{c}\{\{a\},\{b\},\{a, b\},\{a, c\} \\
\{b, c\},\{a, b, c\}\}\end{array}$ & $\{\{c\}\}$ \\
\hline F6 & $\begin{array}{c}\{\{b\},\{a, b\},\{a, c\},\{b, c\},\{a \\
b, c\}\}\end{array}$ & $\begin{array}{c}\{\{b\},\{a, b\},\{a, c\},\{b, c\}, \\
\{a, b, c\}\}\end{array}$ & $\{\{a\},\{c\}\}$ & $\{\{a\},\{b\},\{c\}\}$ \\
\hline F11 & $\{\{a, b\},\{a, c\},\{b, c\},\{a, b, c\}\}$ & $\begin{array}{c}\{\{a, b\},\{a, c\},\{b, c\},\{a, b \\
, c\}\}\end{array}$ & $\{\{a\},\{b\}\}$ & $\{\{b\},\{c\}\}$ \\
\hline F14 & $\{\{a, c\},\{b, c\},\{a, b, c\}\}$ & $\{\{a\},\{b\},\{c\},\{a, b\}\}$ & $\begin{array}{c}\{a \mathrm{a}\},\{b\},\{c\},\{a, b\},\{a, c \\
\},\{b, c\}\}\end{array}$ & $\begin{array}{c}\{\{a\},\{b\},\{c\},\{a, b\},\{a, c\}, \\
\{b, c\},\{a, b, c\}\}\end{array}$ \\
\hline F16 & $\{\{a, c\},\{a, b, c\}\}$ & $\{\{a\},\{b\},\{a, b\},\{b, c\}\}$ & $\begin{array}{c}\{\{a\},\{b\},\{a, b\},\{a, c\},\{b \\
, c\},\{a, b, c\}\}\end{array}$ & $\{\{c\},\{a, b, c\}\}$ \\
\hline F18 & $\{\{a, b, c\}\}$ & $\{\{b\},\{a, b\},\{a, c\},\{b, c\}\}$ & $\{\{a\},\{c\},\{a, c\},\{b, c\}\}$ & $\begin{array}{c}\{\{a\},\{b\},\{c\},\{a, c\},\{b, c\}, \\
\{a, b, c\}\}\end{array}$ \\
\hline - & $\{\Phi\}$ & $\begin{array}{c}\{\{a, b\},\{a, c\},\{b, c\},\{a, b \\
, c\}\}\end{array}$ & $\{\{a\},\{b\},\{a, c\},\{a, b, c\}\}$ & $\{\{b\},\{c\},\{b, c\},\{a, b, c\}\}$ \\
\hline
\end{tabular}


Filter chain \# 14 and its STRT spectrum

\begin{tabular}{|c|c|c|c|c|}
\hline F1 & $\begin{array}{l}\{\{a\},\{b\},\{c\},\{a, b\},\{a, c\} \\
\{b, c\},\{a, b, c\}\}\end{array}$ & $\begin{array}{l}\{\{a\},\{b\},\{c\},\{a, b\},\{a, c\} \\
\{b, c\},\{a, b, c\}\}\end{array}$ & $\begin{array}{l}\{\{a\},\{b\},\{c\},\{a, b\},\{a, c\} \\
\{b, c\},\{a, b, c\}\}\end{array}$ & $\begin{array}{l}\{\{a\},\{b\},\{c\},\{a, b\},\{a, c\} \\
\{b, c\},\{a, b, c\}\}\end{array}$ \\
\hline F2 & $\begin{array}{l}\{\{a\},\{b\},\{a, b\},\{a, c\} \\
\{b, c\},\{a, b, c\}\}\end{array}$ & $\begin{array}{l}\{\{a\},\{b\},\{a, b\},\{a, c\} \\
\{b, c\},\{a, b, c\}\}\end{array}$ & $\begin{array}{l}\{a \mathrm{a}\},\{\mathrm{b}\},\{a, b\},\{a, c\} \\
\{b, c\},\{a, b, c\}\}\end{array}$ & $\{\{c\}\}$ \\
\hline F6 & $\begin{array}{l}\{\{b\},\{a, b\},\{a, c\},\{b, c\} \\
\{a, b, c\}\}\end{array}$ & $\begin{array}{l}\{\{b\},\{a, b\},\{a, c\},\{b, c\} \\
\{a, b, c\}\}\end{array}$ & $\{\{a\},\{c\}\}$ & $\{\{a\},\{b\},\{c\}\}$ \\
\hline F11 & $\begin{array}{l}\{\{a, b\},\{a, c\},\{b, c\},\{a, b, c\} \\
\}\end{array}$ & $\begin{array}{l}\{\{a, b\},\{a, c\},\{b, c\},\{a, b, c\} \\
\}\end{array}$ & $\{\{a\},\{b\}\}$ & $\{\{b\},\{c\}\}$ \\
\hline F14 & $\{\{a, c\},\{b, c\},\{a, b, c\}\}$ & $\{\{a\},\{b\},\{c\},\{a, b\}\}$ & $\begin{array}{l}\{\{a\},\{b\},\{c\},\{a, b\},\{a, c\} \\
,\{b, c\}\}\end{array}$ & $\begin{array}{l}\{\{a\},\{b\},\{c\},\{a, b\},\{a, c\} \\
\{b, c\},\{a, b, c\}\}\end{array}$ \\
\hline F17 & $\{\{b, c\},\{a, b, c\}\}$ & $\{\{a\},\{b\},\{a, b\},\{a, c\}\}$ & $\begin{array}{l}\{\{a\},\{b\},\{a, b\},\{a, c\},\{b \\
c\}, \\
\{a, b, c\}\}\end{array}$ & $\{\{c\},\{a, b, c\}\}$ \\
\hline F18 & $\{\{a, b, c\}\}$ & $\{\{b\},\{a, b\},\{a, c\},\{b, c\}\}$ & $\{\{a\},\{c\},\{a, c\},\{b, c\}\}$ & $\begin{array}{l}\{\{a\},\{b\},\{c\},\{a, c\},\{b, c\} \\
\{a, b, c\}\}\end{array}$ \\
\hline- & $\{\Phi\}$ & $\{\{a, b\},\{a, c\},\{b, c\},\{a, b, c\}$ & $\{\{a\},\{b\},\{b, c\},\{a, b, c\}\}$ & $\{\{b\},\{c\},\{a, c\},\{a, b, c\}\}$ \\
\hline
\end{tabular}

Filter chain \# 15 and its STRT spectrum

\begin{tabular}{|l|l|l|l|l|}
\hline F1 & $\begin{array}{l}\{\{a\},\{b\},\{c\},\{a, b\},\{a, c\}, \\
\{b, c\},\{a, b, c\}\}\end{array}$ & $\begin{array}{l}\{\{a\},\{b\},\{c\},\{a, b\},\{a, c \\
\{, \\
\{b, c\},\{a, b, c\}\}\end{array}$ & $\begin{array}{l}\{\{a\},\{b\},\{c\},\{a, b\},\{a, c\}, \\
\{b, c\},\{a, b, c\}\}\end{array}$ & $\begin{array}{l}\{\{a\},\{b\},\{c\},\{a, b\},\{a, \\
c\}, \\
\{b, c\},\{a, b, c\}\}\end{array}$ \\
\hline F2 & $\begin{array}{l}\{\{a\},\{b\},\{a, b\},\{a, c\}, \\
\{b, c\},\{a, b, c\}\}\end{array}$ & $\begin{array}{l}\{\{a\},\{b\},\{a, b\},\{a, c\}, \\
\{b, c\},\{a, b, c\}\}\end{array}$ & $\begin{array}{l}\{\{a\},\{b\},\{a, b\},\{a, c\},\{b, c\}, \\
\{a, b, c\}\}\end{array}$ & $\{\{c\}\}$ \\
\hline F6 & $\begin{array}{l}\{\{b\},\{a, b\},\{a, c\},\{b, c\}, \\
\{a, b, c\}\}\end{array}$ & $\begin{array}{l}\{\{b\},\{a, b\},\{a, c\},\{b, c\}, \\
\{a, b, c\}\}\end{array}$ & $\{\{a\},\{c\}\}$ & $\{\{a\},\{c\},\{a, c\}\}$ \\
\hline F9 & $\{\{b\},\{a, b\},\{b, c\},\{a, b, c\}\}$ & $\begin{array}{l}\{\{b\},\{a, b\},\{b, c\},\{a, b, c \\
\}\}\end{array}$ & $\{\{a\},\{a, c\}\}$ & $\{\{c\},\{a, c\}\}$ \\
\hline F13 & $\{\{a, b\},\{b, c\},\{a, b, c\}\}$ & $\{\{a\},\{b\},\{c\},\{a, c\}\}$ & $\begin{array}{l}\{\{a\},\{b\},\{c\},\{a, b\},\{a, c\},\{b, c \\
\}\}\end{array}$ & $\begin{array}{l}\{\{a\},\{b\},\{c\},\{a, b\},\{a, \\
c\}, \\
\{b, c\},\{a, b, c\}\}\end{array}$ \\
\hline F15 & $\{\{a, b\},\{a, b, c\}\}$ & $\{\{a\},\{b\},\{a, c\},\{b, c\}\}$ & $\begin{array}{l}\{\{a\},\{b\},\{a, b\},\{a, c\},\{b, c\},\{a \\
, b, c\}\}\end{array}$ & $\{\{c\},\{a, b, c\}\}$ \\
\hline F18 & $\{\{a, b, c\}\}$ & $\begin{array}{l}\{\{b\},\{a, b\},\{a, c\},\{b, c\} \\
\}\end{array}$ & $\{\{a\},\{c\},\{a, b\},\{b, c\}\}$ & $\begin{array}{l}\{\{a\},\{c\},\{a, b\},\{a, c\},\{ \\
b, c\}, \\
\{a, b, c\}\}\end{array}$ \\
\hline- & $\{\Phi\}$ & $\begin{array}{l}\{\{b\},\{a, b\},\{b, c\},\{a, b, c \\
\}\}\end{array}$ & $\{\{a\},\{a, b\},\{a, c\},\{a, b, c\}\}$ & $\begin{array}{l}\{\{c\},\{a, c\},\{b, c\},\{a, b, c \\
\}\}\end{array}$ \\
\hline
\end{tabular}

Filter chain \# 16 and its STRT spectrum

\begin{tabular}{|c|c|c|c|c|}
\hline $\mathrm{F} 1$ & $\begin{array}{l}\{\{a\},\{b\},\{c\},\{a, b\},\{a, c\} \\
\{b, c\},\{a, b, c\}\}\end{array}$ & $\begin{array}{l}\{\{a\},\{b\},\{c\},\{a, b\},\{a, c \\
\}, \\
\{b, c\},\{a, b, c\}\}\end{array}$ & $\begin{array}{l}\{\{a\},\{b\},\{c\},\{a, b\},\{a, c\} \\
\{b, c\},\{a, b, c\}\}\end{array}$ & $\begin{array}{l}\{\{a\},\{b\},\{c\},\{a, b\},\{a, c\} \\
\{b, c\},\{a, b, c\}\}\end{array}$ \\
\hline F2 & $\begin{array}{l}\{\{a\},\{b\},\{a, b\},\{a, c\}, \\
\{b, c\},\{a, b, c\}\}\end{array}$ & $\begin{array}{l}\{a \mathrm{a}\},\{b\},\{a, b\},\{a, c\} \\
\{b, c\},\{a, b, c\}\}\end{array}$ & $\begin{array}{l}\{\{a\},\{b\},\{a, b\},\{a, c\},\{b, c\} \\
\{a, b, c\}\}\end{array}$ & $\{\{c\}\}$ \\
\hline F6 & $\begin{array}{l}\{\{b\},\{a, b\},\{a, c\},\{b, c\},\{a, \\
b, c\}\}\end{array}$ & $\begin{array}{l}\left\{\begin{array}{l}\{b\},\{a, b\},\{a, c\},\{b, c\} \\
\{a, b, c\}\}\end{array}\right.\end{array}$ & $\{\{a\},\{c\}\}$ & $\{\{a\},\{c\},\{a, c\}\}$ \\
\hline F9 & $\{\{b\},\{a, b\},\{b, c\},\{a, b, c\}\}$ & $\begin{array}{l}\{\{b\},\{a, b\},\{b, c\},\{a, b, c \\
\}\}\end{array}$ & $\{\{a\},\{a, c\}\}$ & $\{\{c\},\{a, c\}\}$ \\
\hline $\begin{array}{l}\mathrm{F} 1 \\
3\end{array}$ & $\{\{a, b\},\{b, c\},\{a, b, c\}\}$ & $\{\{a\},\{b\},\{c\},\{a, c\}\}$ & $\begin{array}{l}\{\{a\},\{b\},\{c\},\{a, b\},\{a, c\},\{b, \\
c\}\}\end{array}$ & $\begin{array}{l}\{\{a\},\{b\},\{c\},\{a, b\},\{a, c\} \\
,\{b, c\},\{a, b, c\}\}\end{array}$ \\
\hline $\begin{array}{l}\mathrm{F} 1 \\
7 \\
\end{array}$ & $\{\{b, c\},\{a, b, c\}\}$ & $\{\{a\},\{b\},\{a, b\},\{a, c\}\}$ & $\begin{array}{l}\{\{a\},\{b\},\{a, b\},\{a, c\},\{b, c\}, \\
\{a, b, c\}\}\end{array}$ & $\{\{c\},\{a, b, c\}\}$ \\
\hline $\begin{array}{l}\mathrm{F} 1 \\
8\end{array}$ & $\{\{a, b, c\}\}$ & $\{\{b\},\{a, b\},\{a, c\},\{b, c\}\}$ & $\{\{a\},\{c\},\{a, b\},\{b, c\}\}$ & $\begin{array}{l}\{\{a\},\{c\},\{a \cdot b\},\{a, c\},\{b, \\
c\},\{a, b, c\}\}\end{array}$ \\
\hline & $\{\Phi\}$ & $\begin{array}{l}\{\{b\},\{a, b\},\{b, c\},\{a, b, c \\
\}\}\end{array}$ & $\{\{a\},\{a, c\},\{b, c\},\{a, b, c\}\}$ & $\begin{array}{l}\{\{c\},\{a, b\},\{a, c\},\{a, b, c\} \\
\}\end{array}$ \\
\hline
\end{tabular}


Filter chain \# 17 and its STRT spectrum

\begin{tabular}{|l|l|l|l|l|}
\hline F1 & $\begin{array}{l}\{\{a\},\{b\},\{c\},\{a, b\},\{a, c\},, \\
\{b, c\},\{a, b, c\}\}\end{array}$ & $\begin{array}{l}\{\{a\},\{b\},\{c\},\{a, b\},\{a, c\}, \\
\{b, c\},\{a, b, c\}\}\end{array}$ & $\begin{array}{l}\{\{a\},\{b\},\{c\},\{a, b\},\{a, c\}, \\
\{b, c\},\{a, b, c\}\}\end{array}$ & $\begin{array}{l}\{\{a\},\{b\},\{c\},\{a, b\},\{a, c\}, \\
\{b, c\},\{a, b, c\}\}\end{array}$ \\
\hline F3 & $\begin{array}{l}\{\{a\},\{c\},\{a, b\},\{a, c\}, \\
\{b, c\},\{a, b, c\}\}\end{array}$ & $\begin{array}{l}\{\{a\},\{c\},\{a, b\},\{a, c\}, \\
\{b, c\},\{a, b, c\}\}\end{array}$ & $\begin{array}{l}\{\{a\},\{c\},\{a, b\},\{a, c\}, \\
\{b, c\},\{a, b, c\}\}\end{array}$ & $\{\{b\}\}$ \\
\hline F5 & $\begin{array}{l}\{\{a\},\{a, b\},\{a, c\},\{b, c\}, \\
\{a, b, c\}\}\end{array}$ & $\begin{array}{l}\{\{a\},\{a, b\},\{a, c\},\{b, c\}, \\
\{a, b, c\}\}\end{array}$ & $\{\{b\},\{c\}\}$ & $\{\{b\},\{c\},\{b, c\}\}$ \\
\hline F8 & $\{\{a\},\{a, b\},\{a, c\},\{a, b, c\}\}$ & $\{\{a\},\{a, b\},\{a, c\},\{a, b, c\}\}$ & $\{\{c\},\{b, c\}\}$ & $\{\{b\},\{b, c\}\}$ \\
\hline F12 & $\{\{a, b\},\{a, c\},\{a, b, c\}\}$ & $\{\{a\},\{b\},\{c\},\{b, c\}\}$ & $\begin{array}{l}\{\{a\},\{b\},\{c\},\{a, b\},\{a, c\},\{ \\
b, c\}\}\end{array}$ & $\begin{array}{l}\{\{a\},\{b\},\{c\},\{a, b\},\{a, c\}, \\
\{b, c\},\{a, b, c\}\}\end{array}$ \\
\hline F15 & $\{\{a, b\},\{a, b, c\}\}$ & $\{\{a\},\{c\},\{a, c\},\{b, c\}\}$ & $\begin{array}{l}\{\{a\},\{c\},\{a, b\},\{a, c\},\{b, c\} \\
\{a, b, c\}\}\end{array}$ & $\{\{b\},\{a, b, c\}\}$ \\
\hline F18 & $\{\{a, b, c\}\}$ & $\{\{a\},\{a, b\},\{a, c\},\{b, c\}\}$ & $\{\{b\},\{c\},\{a, b\},\{a, c\}\}$ & $\begin{array}{l}\{\{b\},\{c\},\{a, b\},\{b, c\},\{a, c\} \\
\{a, b, c\}\}\end{array}$ \\
\hline- & $\{\Phi\}$ & $\{\{a\},\{a, b\},\{a, c\},\{a, b, c\}\}$ & $\{\{c\},\{a, b\},\{b, c\},\{a, b, c\}\}$ & $\{\{b\},\{a, c\},\{b, c\},\{a, b, c\}\}$ \\
\hline
\end{tabular}

Filter chain \# 18 and its STRT spectrum

\begin{tabular}{|c|c|c|c|c|}
\hline $\mathrm{F} 1$ & $\begin{array}{c}\{\{a\},\{b\},\{c\},\{a, b\},\{a, c \\
\}, \\
\{b, c\},\{a, b, c\}\}\end{array}$ & $\begin{array}{c}\{\{a\},\{b\},\{c\},\{a, b\},\{a, c\} \\
\{b, c\},\{a, b, c\}\}\end{array}$ & $\begin{array}{c}\{\{a\},\{b\},\{c\},\{a, b\},\{a, c\} \\
\{b, c\},\{a, b, c\}\}\end{array}$ & $\begin{array}{c}\{\{a\},\{b\},\{c\},\{a, b\},\{a, c \\
\}, \\
\{b, c\},\{a, b, c\}\}\end{array}$ \\
\hline F3 & $\begin{array}{c}\{\{a\},\{c\},\{a, b\},\{a, c\} \\
\{b, c\},\{a, b, c\}\}\end{array}$ & $\begin{array}{c}\{\{a\},\{c\},\{a, b\},\{a, c\} \\
\{b, c\},\{a, b, c\}\}\end{array}$ & $\begin{array}{c}\{\{a\},\{c\},\{a, b\},\{a, c\}, \\
\{b, c\},\{a, b, c\}\}\end{array}$ & $\{\{b\}\}$ \\
\hline F5 & $\begin{array}{c}\{\{a\},\{a, b\},\{a, c\},\{b, c\},\{ \\
a, b, c\}\}\end{array}$ & $\begin{array}{r}\{a\},\{a, b\},\{a, c\},\{b, c\},\{a, b \\
, c\}\}\end{array}$ & $\{\{b\},\{c\}\}$ & $\{\{b\},\{c\},\{b, c\}\}$ \\
\hline F8 & $\{\{a\},\{a, b\},\{a, c\},\{a, b, c\}$ & $\{\{a\},\{a, b\},\{a, c\},\{a, b, c\}\}$ & $\{\{c\},\{b, c\}\}$ & $\{\{b\},\{b, c\}\}$ \\
\hline F12 & $\{\{a, b\},\{a, c\},\{a, b, c\}\}$ & $\{\{a\},\{b\},\{c\},\{b, c\}\}$ & $\begin{array}{c}\{\{a\},\{b\},\{c\},\{a, b\},\{a, c\},\{b \\
c\}\}\end{array}$ & $\begin{array}{c}\{\{a\},\{b\},\{c\},\{a, b\},\{a, c \\
\}, \\
\{b, c\},\{a, b, c\}\}\end{array}$ \\
\hline F16 & $\{\{a, c\},\{a, b, c\}\}$ & $\{\{a\},\{c\},\{a, b\},\{b, c\}\}$ & $\begin{array}{c}\{\{a\},\{c\},\{a, b\},\{a, c\},\{b, c\} \\
\{a, b, c\}\}\end{array}$ & $\{\{b\},\{a, b, c\}\}$ \\
\hline F18 & $\{\{a, b, c\}\}$ & $\{\{a\},\{a, b\},\{a, c\},\{b, c\}\}$ & $\{\{b\},\{c\},\{a, b\},\{a, c\}\}$ & $\begin{array}{c}\{\{b\},\{c\},\{a \cdot b\},\{a, c\} \\
\{b, c\},\{a, b, c\}\}\end{array}$ \\
\hline- & $\{\Phi\}$ & $\{\{a\},\{a, b\},\{a, c\},\{a, b, c\}\}$ & $\{\{c\},\{a, c\},\{b, c\},\{a, b, c\}\}$ & $\begin{array}{c}\{\{b\},\{a, b\},\{b, c\},\{a, b, c \\
\}\}\end{array}$ \\
\hline
\end{tabular}

Filter chain \# 19 and its STRT spectrum

\begin{tabular}{|l|l|l|l|l|}
\hline F1 & $\begin{array}{l}\{\{a\},\{b\},\{c\},\{a, b\},\{a, c\}, \\
\{b, c\},\{a, b, c\}\}\end{array}$ & $\begin{array}{l}\{\{a\},\{b\},\{c\},\{a, b\},\{a, \\
c\}, \\
\{b, c\},\{a, b, c\}\}\end{array}$ & $\begin{array}{l}\{\{a\},\{b\},\{c\},\{a, b\},\{a, c\}, \\
\{b, c\},\{a, b, c\}\}\end{array}$ & $\begin{array}{l}\{\{a\},\{b\},\{c\},\{a, b\},\{a, c\}, \\
\{b, c\},\{a, b, c\}\}\end{array}$ \\
\hline F3 & $\begin{array}{l}\{\{a\},\{c\},\{a, b\},\{a, c\}, \\
\{b, c\},\{a, b, c\}\}\end{array}$ & $\begin{array}{l}\{\{a\},\{c\},\{a, b\},\{a, c\}, \\
\{b, c\},\{a, b, c\}\}\end{array}$ & $\begin{array}{l}\{\{a\},\{c\},\{a, b\},\{a, c\},\{b, c\}, \\
\{a, b, c\}\}\end{array}$ & $\{\{b\}\}$ \\
\hline F5 & $\begin{array}{l}\{\{a\},\{a, b\},\{a, c\},\{b, c\}, \\
\{a, b, c\}\}\end{array}$ & $\begin{array}{l}\{\{a\},\{a, b\},\{a, c\},\{b, c\}, \\
\{a, b, c\}\}\end{array}$ & $\{\{b\},\{c\}\}$ & $\{\{a\},\{b\},\{c\}\}$ \\
\hline F11 & $\begin{array}{l}\{\{a, b\},\{a, c\},\{b, c\},\{a, b, c\} \\
\}\end{array}$ & $\begin{array}{l}\{\{a, b\},\{a, c\},\{b, c\},\{a, b \\
, c\}\}\end{array}$ & $\{\{a\},\{c\}\}$ & $\{\{a\},\{b\}\}$ \\
\hline F12 & $\{\{a, b\},\{a, c\},\{a, b, c\}\}$ & $\{\{a\},\{b\},\{c\},\{b, c\}\}$ & $\begin{array}{l}\{\{a\},\{b\},\{c\},\{a, b\},\{a, c\},\{b, \\
c\}\}\end{array}$ & $\begin{array}{l}\{\{a\},\{b\},\{c\},\{a, b\},\{a, c\}, \\
\{b, c\},\{a, b, c\}\}\end{array}$ \\
\hline F15 & $\{\{a, b\},\{a, b, c\}\}$ & $\{\{a\},\{c\},\{a, c\},\{b, c\}\}$ & $\begin{array}{l}\{\{a\},\{c\},\{a, b\},\{a, c\},\{b, c\}, \\
\{a, b, c\}\}\end{array}$ & $\{\{b\},\{a, b, c\}\}$ \\
\hline F18 & $\{\{a, b, c\}\}$ & $\begin{array}{l}\{\{a\},\{a, b\},\{a, c\},\{b, c\} \\
\}\end{array}$ & $\{\{b\},\{c\},\{a, b\},\{a, c\}\}$ & $\begin{array}{l}\{\{a\},\{b\},\{c\},\{a, b\},\{a, c\}, \\
\{a, b, c\}\}\end{array}$ \\
\hline- & $\{\Phi\}$ & $\begin{array}{l}\{\{a, b\},\{a, c\},\{b, c\},\{a, b \\
, c\}\}\end{array}$ & $\{\{a\},\{c\},\{a, b\},\{a, b, c\}\}$ & $\{\{a\},\{b\},\{a, c\},\{a, b, c\}\}$ \\
\hline
\end{tabular}

Filter chain \# 20 and its STRT spectrum

\begin{tabular}{|l|l|l|l|l|}
\hline $\mathrm{F} 1$ & $\begin{array}{l}\{\{a\},\{b\},\{c\},\{a, b\},\{a, c \\
\}, \\
\{b, c\},\{a, b, c\}\}\end{array}$ & $\begin{array}{l}\{\{a\},\{b\},\{c\},\{a, b\},\{a, c\}, \\
\{b, c\},\{a, b, c\}\}\end{array}$ & $\begin{array}{l}\{\{a\},\{b\},\{c\},\{a, b\},\{a, c\}, \\
\{b, c\},\{a, b, c\}\}\end{array}$ & $\begin{array}{l}\{\{a\},\{b\},\{c\},\{a, b\},\{a, c\}, \\
\{b, c\},\{a, b, c\}\}\end{array}$ \\
\hline
\end{tabular}




\begin{tabular}{|l|l|l|l|l|}
\hline F3 & $\begin{array}{l}\{\{a\},\{c\},\{a, b\},\{a, c\}, \\
\{b, c\},\{a, b, c\}\}\end{array}$ & $\begin{array}{l}\{\{a\},\{c\},\{a, b\},\{a, c\},\{b, c \\
\{a, b, c\}\}\end{array}$ & $\begin{array}{l}\{\{a\},\{c\},\{a, b\},\{a, c\},\{b, c \\
\}, \\
\{a, b, c\}\}\end{array}$ & $\{\{b\}\}$ \\
\hline F5 & $\begin{array}{l}\{\{a\},\{a, b\},\{a, c\},\{b, c\}, \\
\{a, b, c\}\}\end{array}$ & $\begin{array}{l}\{\{a\},\{a, b\},\{a, c\},\{b, c\}, \\
\{a, b, c\}\}\end{array}$ & $\{\{b\},\{c\}\}$ & $\{\{a\},\{b\},\{c\}\}$ \\
\hline F11 & $\begin{array}{l}\{\{a, b\},\{a, c\},\{b, c\},\{a, b, \\
c\}\}\end{array}$ & $\begin{array}{l}\{\{a, b\},\{a, c\},\{b, c\},\{a, b, c \\
\}\}\end{array}$ & $\{\{a\},\{c\}\}$ & $\{\{a\},\{b\}\}$ \\
\hline F12 & $\{\{a, b\},\{a, c\},\{a, b, c\}\}$ & $\{\{a\},\{b\},\{c\},\{b, c\}\}$ & $\begin{array}{l}\{\{a\},\{b\},\{c\},\{a, b\},\{a, c\}, \\
\{b, c\}\}\end{array}$ & $\begin{array}{l}\{\{a\},\{b\},\{c\},\{a, b\},\{a, c\}, \\
\{b, c\},\{a, b, c\}\}\end{array}$ \\
\hline$F 16$ & $\{\{a, c\},\{a, b, c\}\}$ & $\{\{a\},\{c\},\{a, b\},\{b, c\}\}$ & $\begin{array}{l}\{\{a\},\{c\},\{a, b\},\{a, c\},\{b, c \\
\{a, b, c\}\}\end{array}$ & $\{\{b\},\{a, b, c\}\}$ \\
\hline F18 & $\{\{a, b, c\}\}$ & $\{\{a\},\{a, b\},\{a, c\},\{b, c\}\}$ & $\{\{b\},\{c\},\{a, b\},\{a, c\}\}$ & $\begin{array}{l}\{\{a\},\{b\},\{c\},\{a, b\},\{a, c\}, \\
\{a, b, c\}\}\end{array}$ \\
\hline- & $\{\Phi\}$ & $\begin{array}{l}\{\{a, b\},\{a, c\},\{b, c\},\{a, b, c \\
\}\}\end{array}$ & $\{\{a\},\{c\},\{a, c\},\{a, b, c\}\}$ & $\{\{a\},\{b\},\{a, b\},\{a, b, c\}\}$ \\
\hline
\end{tabular}

Filter chain \# 21 and its STRT spectrum

\begin{tabular}{|l|l|l|l|l|}
\hline F1 & $\begin{array}{l}\{\{a\},\{b\},\{c\},\{a, b\},\{a, c\}, \\
\{b, c\},\{a, b, c\}\}\end{array}$ & $\begin{array}{l}\{\{a\},\{b\},\{c\},\{a, b\},\{a, c\}, \\
\{b, c\},\{a, b, c\}\}\end{array}$ & $\begin{array}{l}\{\{a\},\{b\},\{c\},\{a, b\},\{a, c\}, \\
\{b, c\},\{a, b, c\}\}\end{array}$ & $\begin{array}{l}\{a \mathrm{a}\},\{b\},\{c\},\{a, b\},\{a, c\}, \\
\{b, c\},\{a, b, c\}\}\end{array}$ \\
\hline F3 & $\begin{array}{l}\{\{a\},\{c\},\{a, b\},\{a, c\}, \\
\{b, c\},\{a, b, c\}\}\end{array}$ & $\begin{array}{l}\{\{a\},\{c\},\{a, b\},\{a, c\}, \\
\{b, c\},\{a, b, c\}\}\end{array}$ & $\begin{array}{l}\{\{a\},\{c\},\{a, b\},\{a, c\}, \\
\{b, c\},\{a, b, c\}\}\end{array}$ & $\{\{b\}\}$ \\
\hline F5 & $\begin{array}{l}\{\{a\},\{a, b\},\{a, c\},\{b, c\}, \\
\{a, b, c\}\}\end{array}$ & $\begin{array}{l}\{\{a\},\{a, b\},\{a, c\},\{b, c\}, \\
\{a, b, c\}\}\end{array}$ & $\{\{b\},\{c\}\}$ & $\{\{a\},\{b\},\{c\}\}$ \\
\hline$F 11$ & $\begin{array}{l}\{\{a, b\},\{a, c\},\{b, c\},\{a, b, c\} \\
\{\end{array}$ & $\begin{array}{l}\{\{a, b\},\{a, c\},\{b, c\},\{a, b, c\} \\
\}\end{array}$ & $\{\{a\},\{c\}\}$ & $\{\{a\},\{b\}\}$ \\
\hline$F 13$ & $\{\{a, b\},\{b, c\},\{a, b, c\}\}$ & $\{\{a\},\{b\},\{c\},\{a, c\}\}$ & $\begin{array}{l}\{\{a\},\{b\},\{c\},\{a, b\},\{a, c\}, \\
\{b, c\}\}\end{array}$ & $\begin{array}{l}\{\{a\},\{b\},\{c\},\{a, b\},\{a, c\}, \\
\{b, c\},\{a, b, c\}\}\end{array}$ \\
\hline F15 & $\{\{a, b\},\{a, b, c\}\}$ & $\{\{a\},\{c\},\{a, c\},\{b, c\}\}$ & $\begin{array}{l}\{\{a\},\{c\},\{a, b\},\{a, c\},\{b, c\}, \\
\{a, b, c\}\}\end{array}$ & $\{\{b\},\{a, b, c\}\}$ \\
\hline F18 & $\{\{a, b, c\}\}$ & $\{\{a\},\{a, b\},\{a, c\},\{b, c\}\}$ & $\{\{b\},\{c\},\{a, b\},\{b, c\}\}$ & $\begin{array}{l}\{a\},\{b\},\{c\},\{a, b\},\{b, c\}, \\
\{a, b, c\}\}\end{array}$ \\
\hline- & $\{\Phi\}$ & $\begin{array}{l}\{\{a, b\},\{a, c\},\{b, c\},\{a, b, c\} \\
\}\end{array}$ & $\{a a\},\{c\},\{a, b\},\{a, b, c\}\}$ & $\{\{a\},\{b\},\{b, c\},\{a, b, c\}\}$ \\
\hline
\end{tabular}

Filter chain \# 22 and its STRT spectrum

\begin{tabular}{|l|l|l|l|l|}
\hline F1 & $\begin{array}{l}\{\{a\},\{b\},\{c\},\{a, b\},\{a, c \\
\}, \\
\{b, c\},\{a, b, c\}\}\end{array}$ & $\begin{array}{l}\{\{a\},\{b\},\{c\},\{a, b\},\{a, c \\
\{b, c\},\{a, b, c\}\}\end{array}$ & $\begin{array}{l}\{\{a\},\{b\},\{c\},\{a, b\},\{a, c\}, \\
\{b, c\},\{a, b, c\}\}\end{array}$ & $\begin{array}{l}\{\{a\},\{b\},\{c\},\{a, b\},\{a, c\}, \\
\{b, c\},\{a, b, c\}\}\end{array}$ \\
\hline F3 & $\begin{array}{l}\{\{a\},\{c\},\{a, b\},\{a, c\}, \\
\{b, c\},\{a, b, c\}\}\end{array}$ & $\begin{array}{l}\{\{a\},\{c\},\{a, b\},\{a, c\}, \\
\{b, c\},\{a, b, c\}\}\end{array}$ & $\begin{array}{l}\{\{a\},\{c\},\{a, b\},\{a, c\}, \\
\{b, c\},\{a, b, c\}\}\end{array}$ & $\{\{b\}\}$ \\
\hline F5 & $\begin{array}{l}\{\{a\},\{a, b\},\{a, c\},\{b, c\}, \\
\{a, b, c\}\}\end{array}$ & $\begin{array}{l}\{\{a\},\{a, b\},\{a, c\},\{b, c\}, \\
\{a, b, c\}\}\end{array}$ & $\{\{b\},\{c\}\}$ & $\{\{a\},\{b\},\{c\}\}$ \\
\hline F11 & $\begin{array}{l}\{\{a, b\},\{a, c\},\{b, c\},\{a, b, \\
c\}\}\end{array}$ & $\begin{array}{l}\{\{a, b\},\{a, c\},\{b, c\},\{a, b, \\
c\}\}\end{array}$ & $\{\{a\},\{c\}\}$ & $\{\{a\},\{b\}\}$ \\
\hline F13 & $\{\{a, b\},\{b, c\},\{a, b, c\}\}$ & $\{\{a\},\{b\},\{c\},\{a, c\}\}$ & $\begin{array}{l}\{\{a\},\{b\},\{c\},\{a, b\},\{a, c\},\{b, c \\
\}\}\end{array}$ & $\begin{array}{l}\{a b\},\{b\},\{c\},\{a, b\},\{a, c\}, \\
\{b, c\},\{a, b, c\}\}\end{array}$ \\
\hline F17 & $\{\{b, c\},\{a, b, c\}\}$ & $\{\{a\},\{c\},\{a, b\},\{a, c\}\}$ & $\begin{array}{l}\{a b\},\{c\},\{a, b\},\{a, c\}, \\
\{b, c\},\{a, b, c\}\}\end{array}$ & $\{\{b\},\{a, b, c\}\}$ \\
\hline F18 & $\{\{a, b, c\}\}$ & $\{\{a\},\{a, b\},\{a, c\},\{b, c\}\}$ & $\{\{b\},\{c\},\{a, b\},\{b, c\}\}$ & $\begin{array}{l}\{a\},,\{b\},\{c\},\{a, b\},\{b, c\}, \\
\{a, b, c\}\}\end{array}$ \\
\hline- & $\{\Phi\}$ & $\begin{array}{l}\{\{a, b\},\{a, c\},\{b, c\},\{a, b, \\
c\}\}\end{array}$ & $\{\{a\},\{c\},\{b, c\},\{a, b, c\}\}$ & $\{\{a\},\{b\},\{a, b\},\{a, b, c\}\}$ \\
\hline
\end{tabular}

Filter chain \# 23 and its STRT spectrum

\begin{tabular}{|c|c|c|c|c|}
\hline F1 & $\begin{array}{l}\{\{a\},\{b\},\{c\},\{a, b\},\{a, c \\
\} \\
\{b, c\},\{a, b, c\}\}\end{array}$ & $\begin{array}{l}\{\{a\},\{b\},\{c\},\{a, b\},\{a, c\} \\
\{b, c\},\{a, b, c\}\}\end{array}$ & $\begin{array}{l}\{\{a\},\{b\},\{c\},\{a, b\},\{a, c\} \\
\{b, c\},\{a, b, c\}\}\end{array}$ & $\begin{array}{l}\{\{a\},\{b\},\{c\},\{a, b\},\{a, c\}, \\
\{b, c\},\{a, b, c\}\}\end{array}$ \\
\hline F3 & $\begin{array}{l}\{a \mathrm{a}\},\{c\},\{a, b\},\{a, c\} \\
\{b, c\},\{a, b, c\}\}\end{array}$ & $\begin{array}{l}\{a \mathrm{a}\},\{c\},\{a, b\},\{a, c\} \\
\{b, c\},\{a, b, c\}\}\end{array}$ & $\begin{array}{l}\{\{a\},\{c\},\{a, b\},\{a, c\}, \\
\{b, c\},\{a, b, c\}\}\end{array}$ & $\{\{b\}\}$ \\
\hline
\end{tabular}




\begin{tabular}{|l|l|l|l|l|}
\hline F5 & $\begin{array}{l}\{\{a\},\{a, b\},\{a, c\},\{b, c\}, \\
\{a, b, c\}\}\end{array}$ & $\begin{array}{l}\{\{a\},\{a, b\},\{a, c\},\{b, c\}, \\
\{a, b, c\}\}\end{array}$ & $\{\{b\},\{c\}\}$ & $\{\{a\},\{b\},\{c\}\}$ \\
\hline F11 & $\begin{array}{l}\{a, b\},\{a, c\},\{b, c\},\{a, b, \\
c\}\}\end{array}$ & $\begin{array}{l}\{\{a, b\},\{a, c\},\{b, c\},\{a, b, \\
c\}\}\end{array}$ & $\{\{a\},\{c\}\}$ & $\{\{a\},\{b\}\}$ \\
\hline F14 & $\{\{a, c\},\{b, c\},\{a, b, c\}\}$ & $\{\{a\},\{b\},\{c\},\{a, b\}\}$ & $\begin{array}{l}\{\{a\},\{b\},\{c\},\{a, b\},\{a, c\},\{b, c \\
\}\}\end{array}$ & $\begin{array}{l}\{\{a\},\{b\},\{c\},\{a, b\},\{a, c\}, \\
\{b, c\},\{a, b, c\}\}\end{array}$ \\
\hline F16 & $\{\{a, c\},\{a, b, c\}\}$ & $\{\{a\},\{c\},\{a, b\},\{b, c\}\}$ & $\begin{array}{l}\{\{a\},\{c\},\{a, b\},\{a, c\},\{b, c\}, \\
\{a, b, c\}\}\end{array}$ & $\{\{b\},\{a, b, c\}\}$ \\
\hline F18 & $\{\{a, b, c\}\}$ & $\{\{a\},\{a, b\},\{a, c\},\{b, c\}\}$ & $\{\{b\},\{c\},\{a, c\},\{b, c\}\}$ & $\begin{array}{l}\{\{a\},\{b\},\{c\},\{a, c\},\{b, c\}, \\
\{a, b, c\}\}\end{array}$ \\
\hline- & $\{\Phi\}$ & $\begin{array}{l}\{\{a, b\},\{a, c\},\{b, c\},\{a, b, \\
c\}\}\end{array}$ & $\{\{a\},\{c\},\{a, c\},\{a, b, c\}\}$ & $\{\{a\},\{b\},\{b, c\},\{a, b, c\}\}$ \\
\hline
\end{tabular}

Filter chain \# 24 and its STRT spectrum

\begin{tabular}{|c|c|c|c|c|}
\hline $\mathrm{F} 1$ & $\begin{array}{l}\{\{a\},\{b\},\{c\},\{a, b\},\{a, c \\
\}, \\
\{b, c\},\{a, b, c\}\}\end{array}$ & $\begin{array}{l}\{\{a\},\{b\},\{c\},\{a, b\},\{a, c\} \\
\{b, c\},\{a, b, c\}\}\end{array}$ & $\begin{array}{l}\{a \mathrm{a}\},\{b\},\{c\},\{a, b\},\{a, c\} \\
\{b, c\},\{a, b, c\}\}\end{array}$ & $\begin{array}{l}\{\{a\},\{b\},\{c\},\{a, b\},\{a, c\}, \\
\{b, c\},\{a, b, c\}\}\end{array}$ \\
\hline F3 & $\begin{array}{l}\{\{a\},\{c\},\{a, b\},\{a, c\} \\
\{b, c\},\{a, b, c\}\}\end{array}$ & $\begin{array}{l}\{\{a\},\{c\},\{a, b\},\{a, c\} \\
\{b, c\},\{a, b, c\}\}\end{array}$ & $\begin{array}{l}\{\{a\},\{c\},\{a, b\},\{a, c\},\{b, c\}, \\
\{a, b, c\}\}\end{array}$ & $\{\{\mathrm{b}\}\}$ \\
\hline F5 & $\begin{array}{l}\{\{a\},\{a, b\},\{a, c\},\{b, c\}, \\
\{a, b, c\}\}\end{array}$ & $\begin{array}{l}\{\{a\},\{a, b\},\{a, c\},\{b, c\},\{ \\
a, b, c\}\}\end{array}$ & $\{\{b\},\{c\}\}$ & $\{\{a\},\{b\},\{c\}\}$ \\
\hline $\mathrm{F} 11$ & $\begin{array}{l}\{\{a, b\},\{a, c\},\{b, c\},\{a, b, \\
c\}\}\end{array}$ & $\begin{array}{l}\{\{a, b\},\{a, c\},\{b, c\},\{a, b, \\
c\}\}\end{array}$ & $\{\{a\},\{c\}\}$ & $\{\{a\},\{b\}\}$ \\
\hline $\mathrm{F} 14$ & $\{\{a, c\},\{b, c\},\{a, b, c\}\}$ & $\{\{a\},\{b\},\{c\},\{a, b\}\}$ & $\begin{array}{l}\{\{a\},\{b\},\{c\},\{a, b\},\{a, c\},\{b, c \\
\}\}\end{array}$ & $\begin{array}{l}\{\{a\},\{b\},\{c\},\{a, b\},\{a, c\}, \\
\{b, c\},\{a, b, c\}\}\end{array}$ \\
\hline F17 & $\{\{b, c\},\{a, b, c\}\}$ & $\{\{a\},\{c\},\{a, b\},\{a, c\}\}$ & $\begin{array}{l}\{\{a\},\{c\},\{a, b\},\{a, c\},\{b, c\}, \\
\{a, b, c\}\}\end{array}$ & $\{\{b\},\{a, b, c\}\}$ \\
\hline F18 & $\{\{a, b, c\}\}$ & $\{\{a\},\{a, b\},\{a, c\},\{b, c\}\}$ & $\{\{b\},\{c\},\{a, c\},\{b, c\}\}$ & $\begin{array}{l}\{\{a\},\{b\},\{c\},\{a \cdot c\},\{b, c\}, \\
\{a, b, c\}\}\end{array}$ \\
\hline- & $\{\Phi\}$ & $\begin{array}{l}\{\{a, b\},\{a, c\},\{b, c\},\{a, b, \\
c\}\}\end{array}$ & $\{\{a\},\{c\},\{b, c\},\{a, b, c\}\}$ & $\{\{a\},\{b\},\{a, c\},\{a, b, c\}\}$ \\
\hline
\end{tabular}

Filter chain \# 25 and its STRT spectrum

\begin{tabular}{|c|c|c|c|c|}
\hline $\mathrm{F} 1$ & $\begin{array}{l}\{\{a\},\{b\},\{c\},\{a, b\},\{a, c\} \\
\{b, c\},\{a, b, c\}\}\end{array}$ & $\begin{array}{l}\{\{a\},\{b\},\{c\},\{a, b\},\{a, c\} \\
\{b, c\},\{a, b, c\}\}\end{array}$ & $\begin{array}{l}\{\{a\},\{b\},\{c\},\{a, b\},\{a, c\} \\
\{b, c\},\{a, b, c\}\}\end{array}$ & $\begin{array}{l}\{a \mathrm{a}\},\{b\},\{c\},\{a, b\},\{a, c\} \\
\{b, c\},\{a, b, c\}\}\end{array}$ \\
\hline F3 & $\begin{array}{l}\{\{a\},\{c\},\{a, b\},\{a, c\} \\
\{b, c\},\{a, b, c\}\}\end{array}$ & $\begin{array}{l}\{\{a\},\{c\},\{a, b\},\{a, c\},\{b, c\}, \\
\{a, b, c\}\}\end{array}$ & $\begin{array}{l}\{\{a\},\{c\},\{a, b\},\{a, c\},\{b, c\}, \\
\{a, b, c\}\}\end{array}$ & $\{\{b\}\}$ \\
\hline F7 & $\begin{array}{l}\{\{c\},\{a, b\},\{a, c\},\{b, c\}, \\
\{a, b, c\}\}\end{array}$ & $\begin{array}{l}\{\{c\},\{a, b\},\{a, c\},\{b, c\}, \\
\{a, b, c\}\}\end{array}$ & $\{\{a\},\{b\}\}$ & $\{\{a\},\{b\},\{c\}\}$ \\
\hline F11 & $\{\{a, b\},\{a, c\},\{b, c\},\{a, b, c\}\}$ & $\{\{a, b\},\{a, c\},\{b, c\},\{a, b, c\}\}$ & $\{\{a\},\{c\}\}$ & $\{\{b\},\{c\}\}$ \\
\hline F12 & $\{\{a, b\},\{a, c\},\{a, b, c\}\}$ & $\{\{a\},\{b\},\{c\},\{b, c\}\}$ & $\begin{array}{l}\{\{a\},\{b\},\{c\},\{a, b\},\{a, c\} \\
\{b, c\}\}\end{array}$ & $\begin{array}{l}\{\{a\},\{b\},\{c\},\{a, b\},\{a, c\}, \\
\{b, c\},\{a, b, c\}\}\end{array}$ \\
\hline F15 & $\{\{a, b\},\{a, b, c\}\}$ & $\{\{a\},\{c\},\{a, c\},\{b, c\}\}$ & $\begin{array}{l}\{\{a\},\{c\},\{a, b\},\{a, c\},\{b, c\} \\
\{a, b, c\}\}\end{array}$ & $\{\{b\},\{a, b, c\}\}$ \\
\hline F18 & $\{\{a, b, c\}\}$ & $\{\{c\},\{a, b\},\{a, c\},\{b, c\}\}$ & $\{\{a\},\{b\},\{a, b\},\{a, c\}\}$ & $\begin{array}{l}\{\{a\},\{b\},\{c\},\{a, b\} \\
\{a, c\},\{a, b, c\}\}\end{array}$ \\
\hline- & $\{\Phi\}$ & $\{\{a, b\},\{a, c\},\{b, c\},\{a, b, c\}\}$ & $\{\{a\},\{c\},\{a, b\},\{a, b, c\}\}$ & $\{\{b\},\{c\},\{a, c\},\{a, b, c\}\}$ \\
\hline
\end{tabular}

Filter chain \# 26 and its STRT spectrum

\begin{tabular}{|l|l|l|l|l|}
\hline \multirow{2}{*}{ F1 } & $\{\{a\},\{b\},\{c\},\{a, b\},\{a, c\}$, & $\{\{a\},\{b\},\{c\},\{a, b\},\{a, c\}$, & $\{\{a\},\{b\},\{c\},\{a, b\},\{a, c\}$, & $\{\{a\},\{b\},\{c\},\{a, b\},\{a, c\}$, \\
& $\{b, c\},\{a, b, c\}\}$ & $\{b, c\},\{a, b, c\}\}$ & $\{b, c\},\{a, b, c\}\}$ & $\{b, c\},\{a, b, c\}\}$ \\
\hline \multirow{2}{*}{ F3 } & $\{\{a\},\{c\},\{a, b\},\{a, c\}$, & $\{\{a\},\{c\},\{a, b\},\{a, c\}$, & $\{a\},\{c\},\{a, b\},\{a, c\},\{b, c\}$, & $\{\{b\}\}$ \\
& $\{b, c\},\{a, b, c\}\}$ & $\{b, c\},\{a, b, c\}\}$ & $\{a, b, c\}\}$ & $\{b$, \\
\hline
\end{tabular}




\begin{tabular}{|l|l|l|l|l|}
\hline F7 & $\begin{array}{l}\{\{c\},\{a, b\},\{a, c\},\{b, c\}, \\
\{a, b, c\}\}\end{array}$ & $\begin{array}{l}\{\{c\},\{a, b\},\{a, c\},\{b, c\}, \\
\{a, b, c\}\}\end{array}$ & $\{\{a\},\{b\}\}$ & $\{\{a\},\{b\},\{c\}\}$ \\
\hline F11 & $\{\{a, b\},\{a, c\},\{b, c\},\{a, b, c\}\}$ & $\{\{a, b\},\{a, c\},\{b, c\},\{a, b, c\}\}$ & $\{\{a\},\{c\}\}$ & $\{\{b\},\{c\}\}$ \\
\hline F12 & $\{\{a, b\},\{a, c\},\{a, b, c\}\}$ & $\{\{a\},\{b\},\{c\},\{b, c\}\}$ & $\begin{array}{l}\{a a\},\{b\},\{c\},\{a, b\},\{a, c\}, \\
\{b, c\}\}\end{array}$ & $\begin{array}{l}\{\{a\},\{b\},\{c\},\{a, b\},\{a, c\}, \\
\{b, c\},\{a, b, c\}\}\end{array}$ \\
\hline$F 16$ & $\{\{a, c\},\{a, b, c\}\}$ & $\{\{a\},\{c\},\{a, b\},\{b, c\}\}$ & $\begin{array}{l}\{\{a\},\{c\},\{a, b\},\{a, c\},\{b, c\}, \\
\{a, b, c\}\}\end{array}$ & $\{\{b\},\{a, b, c\}\}$ \\
\hline F18 & $\{\{a, b, c\}\}$ & $\{\{c\},\{a, b\},\{a, c\},\{b, c\}\}$ & $\{\{a\},\{b\},\{a, b\},\{a, c\}\}$ & $\begin{array}{l}\{\{a\},\{b\},\{c\},\{a, b\},\{a, c\}, \\
\{a, b, c\}\}\end{array}$ \\
\hline- & $\{\Phi\}$ & $\{\{a, b\},\{a, c\},\{b, c\},\{a, b, c\}\}$ & $\{\{a\},\{c\},\{a, c\},\{a, b, c\}\}$ & $\{\{b\},\{c\},\{a, b\},\{a, b, c\}\}$ \\
\hline
\end{tabular}

Filter chain \# 27 and its STRT spectrum

\begin{tabular}{|l|l|l|l|l|}
\hline F1 & $\begin{array}{l}\{\{a\},\{b\},\{c\},\{a, b\},\{a, c\}, \\
\{b, c\},\{a, b, c\}\}\end{array}$ & $\begin{array}{l}\{\{a\},\{b\},\{c\},\{a, b\},\{a, c\}, \\
\{b, c\},\{a, b, c\}\}\end{array}$ & $\begin{array}{l}\{\{a\},\{b\},\{c\},\{a, b\},\{a, c\}, \\
\{b, c\},\{a, b, c\}\}\end{array}$ & $\begin{array}{l}\{\{a\},\{b\},\{c\},\{a, b\},\{a, c\}, \\
\{b, c\},\{a, b, c\}\}\end{array}$ \\
\hline F3 & $\begin{array}{l}\{\{a\},\{c\},\{a, b\},\{a, c\}, \\
\{b, c\},\{a, b, c\}\}\end{array}$ & $\begin{array}{l}\{\{a\},\{c\},\{a, b\},\{a, c\}, \\
\{b, c\},\{a, b, c\}\}\end{array}$ & $\begin{array}{l}\{\{a\},\{c\},\{a, b\},\{a, c\},\{b, c\}, \\
\{a, b, c\}\}\end{array}$ & $\{\{b\}\}$ \\
\hline F7 & $\begin{array}{l}\{\{c\},\{a, b\},\{a, c\},\{b, c\}, \\
\{a, b, c\}\}\end{array}$ & $\begin{array}{l}\{\{c\},\{a, b\},\{a, c\},\{b, c\}, \\
\{a, b, c\}\}\end{array}$ & $\{\{a\},\{b\}\}$ & $\{\{a\},\{b\},\{c\}\}$ \\
\hline F11 & $\begin{array}{l}\{\{a, b\},\{a, c\},\{b, c\},\{a, b, c\} \\
\}\end{array}$ & $\begin{array}{l}\{\{a, b\},\{a, c\},\{b, c\},\{a, b, c\} \\
\}\end{array}$ & $\{\{a\},\{c\}\}$ & $\{\{b\},\{c\}\}$ \\
\hline F13 & $\{\{a, b\},\{b, c\},\{a, b, c\}\}$ & $\{\{a\},\{b\},\{c\},\{a, c\}\}$ & $\begin{array}{l}\{\{a\},\{b\},\{c\},\{a, b\},\{a, c\}, \\
\{b, c\}\}\end{array}$ & $\begin{array}{l}\{\{a\},\{b\},\{c\},\{a, b\},\{a, c\}, \\
\{b, c\},\{a, b, c\}\}\end{array}$ \\
\hline F15 & $\{\{a, b\},\{a, b, c\}\}$ & $\{\{a\},\{c\},\{a, c\},\{b, c\}\}$ & $\begin{array}{l}\{a a\},\{c\},\{a, b\},\{a, c\},\{b, c\}, \\
\{a, b, c\}\}\end{array}$ & $\{\{b\},\{a, b, c\}\}$ \\
\hline F18 & $\{\{a, b, c\}\}$ & $\{\{c\},\{a, b\},\{a, c\},\{b, c\}\}$ & $\{\{a\},\{b\},\{a, b\},\{b, c\}\}$ & $\begin{array}{l}\{\{a\},\{b\},\{c\},\{a, b\},\{b, c\}, \\
\{a, b, c\}\}\end{array}$ \\
\hline- & $\{\Phi\}$ & $\{\{a, b\},\{a, c\},\{b, c\},\{a, b, c\}\}$ & $\{\{a\},\{c\},\{a, b\},\{a, b, c\}\}$ & $\{\{b\},\{c\},\{b, c\},\{a, b, c\}\}$ \\
\hline
\end{tabular}

Filter chain \# 28 and its STRT spectrum

\begin{tabular}{|c|c|c|c|c|}
\hline F1 & $\begin{array}{l}\{\{a\},\{b\},\{c\},\{a, b\},\{a, c\} \\
\{b, c\},\{a, b, c\}\}\end{array}$ & $\begin{array}{l}\{a \mathrm{a}\},\{b\},\{c\},\{a, b\},\{a, c\} \\
\{b, c\},\{a, b, c\}\}\end{array}$ & $\begin{array}{l}\{\{a\},\{b\},\{c\},\{a, b\},\{a, c\} \\
\{b, c\},\{a, b, c\}\}\end{array}$ & $\begin{array}{l}\{\{a\},\{b\},\{c\},\{a, b\},\{a, c\} \\
\{b, c\},\{a, b, c\}\}\end{array}$ \\
\hline F3 & $\begin{array}{l}\{\{a\},\{c\},\{a, b\},\{a, c\} \\
\{b, c\},\{a, b, c\}\}\end{array}$ & $\begin{array}{l}\{\{a\},\{c\},\{a, b\},\{a, c\} \\
\{b, c\},\{a, b, c\}\}\end{array}$ & $\begin{array}{l}\{\{a\},\{c\},\{a, b\},\{a, c\} \\
\{b, c\},\{a, b, c\}\}\end{array}$ & $\{\{b\}\}$ \\
\hline F7 & $\begin{array}{l}\{\{c\},\{a, b\},\{a, c\},\{b, c\}, \\
\{a, b, c\}\}\end{array}$ & $\begin{array}{l}\{\{c\},\{a, b\},\{a, c\},\{b, c\}, \\
\{a, b, c\}\}\end{array}$ & $\{\{a\},\{b\}\}$ & $\{\{a\},\{b\},\{c\}\}$ \\
\hline F11 & $\{\{a, b\},\{a, c\},\{b, c\},\{a, b, c\}\}$ & $\{\{a, b\},\{a, c\},\{b, c\},\{a, b, c\}\}$ & $\{\{a\},\{c\}\}$ & $\{\{b\},\{c\}\}$ \\
\hline F13 & $\{\{a, b\},\{b, c\},\{a, b, c\}\}$ & $\{\{a\},\{b\},\{c\},\{a, c\}\}$ & $\begin{array}{l}\{\{a\},\{b\},\{c\},\{a, b\},\{a, c\} \\
\{b, c\}\}\end{array}$ & $\begin{array}{l}\{\{a\},\{b\},\{c\},\{a, b\},\{a, c\}, \\
\{b, c\},\{a, b, c\}\}\end{array}$ \\
\hline $\mathrm{F} 17$ & $\{\{b, c\},\{a, b, c\}\}$ & $\{\{a\},\{c\},\{a, b\},\{a, c\}\}$ & $\begin{array}{l}\{\{a\},\{c\},\{a, b\},\{a, c\},\{b, c\}, \\
\{a, b, c\}\}\end{array}$ & $\{\{b\},\{a, b, c\}\}$ \\
\hline F18 & $\{\{a, b, c\}\}$ & $\{\{c\},\{a, b\},\{a, c\},\{b, c\}\}$ & $\{\{a\},\{b\},\{a, b\},\{b, c\}\}$ & $\begin{array}{l}\{\{a\},\{b\},\{c\},\{a, b\},\{b, c\}, \\
\{a, b, c\}\}\end{array}$ \\
\hline - & $\{\Phi\}$ & $\{\{a, b\},\{a, c\},\{b, c\},\{a, b, c\}\}$ & $\{\{a\},\{c\},\{b, c\},\{a, b, c\}\}$ & $\{\{b\},\{c\},\{a, b\},\{a, b, c\}\}$ \\
\hline
\end{tabular}

Filter chain \# 29 and its STRT spectrum

\begin{tabular}{|l|l|l|l|l|}
\hline F1 & $\begin{array}{l}\{\{a\},\{b\},\{c\},\{a, b\},\{a, c\}, \\
\{b, c\},\{a, b, c\}\}\end{array}$ & $\begin{array}{l}\{\{a\},\{b\},\{c\},\{a, b\},\{a, c\}, \\
\{b, c\},\{a, b, c\}\}\end{array}$ & $\begin{array}{l}\{\{a\},\{b\},\{c\},\{a, b\},\{a, c\}, \\
\{b, c\},\{a, b, c\}\}\end{array}$ & $\begin{array}{l}\{\{a\},\{b\},\{c\},\{a, b\},\{a, c\}, \\
\{b, c\},\{a, b, c\}\}\end{array}$ \\
\hline F3 & $\begin{array}{l}\{\{a\},\{c\},\{a, b\},\{a, c\}, \\
\{b, c\},\{a, b, c\}\}\end{array}$ & $\begin{array}{l}\{\{a\},\{c\},\{a, b\},\{a, c\},\{b, c\}, \\
\{a, b, c\}\}\end{array}$ & $\begin{array}{l}\{a a\},\{c\},\{a, b\},\{a, c\},\{b, c\}, \\
\{a, b, c\}\}\end{array}$ & $\{\{b\}\}$ \\
\hline F7 & $\begin{array}{l}\{\{c\},\{a, b\},\{a, c\},\{b, c\}, \\
\{a, b, c\}\}\end{array}$ & $\begin{array}{l}\{\{c\},\{a, b\},\{a, c\},\{b, c\}, \\
\{a, b, c\}\}\end{array}$ & $\{\{a\},\{b\}\}$ & $\{\{a\},\{b\},\{c\}\}$ \\
\hline F11 & $\begin{array}{l}\{\{a, b\},\{a, c\},\{b, c\}, \\
\{a, b, c\}\}\end{array}$ & $\{\{a, b\},\{a, c\},\{b, c\},\{a, b, c\}\}$ & $\{\{a\},\{c\}\}$ & $\{\{b\},\{c\}\}$ \\
\hline \multirow{2}{*}{ F14 } & $\{\{a, c\},\{b, c\},\{a, b, c\}\}$ & $\{\{a\},\{b\},\{c\},\{a, b\}\}$ & $\begin{array}{l}\{\{a\},\{b\},\{c\},\{a, b\},\{a, c\}, \\
\{b, c\}\}\end{array}$ & $\begin{array}{l}\{\{a\},\{b\},\{c\},\{a, b\},\{a, c\}, \\
\{b, c\},\{a, b, c\}\}\end{array}$ \\
\hline
\end{tabular}




\begin{tabular}{|l|l|l|l|l|}
\hline F16 & $\{\{a, c\},\{a, b, c\}\}$ & $\{\{a\},\{c\},\{a, b\},\{b, c\}\}$ & $\begin{array}{l}\{a \mathrm{a}\},\{c\},\{a, b\},\{a, c\},\{b, c\}, \\
\{a, b, c\}\}\end{array}$ & $\{\{b\},\{a, b, c\}\}$ \\
\hline F18 & $\{\{a, b, c\}\}$ & $\{\{c\},\{a, b\},\{a, c\},\{b, c\}\}$ & $\{\{a\},\{b\},\{a, c\},\{b, c\}\}$ & $\begin{array}{l}\{\{a\},\{b\},\{c\},\{a, c\},\{b, c\}, \\
\{a, b, c\}\}\end{array}$ \\
\hline- & $\{\Phi\}$ & $\{\{a, b\},\{a, c\},\{b, c\},\{a, b, c\}\}$ & $\{\{a\},\{c\},\{a, c\},\{a, b, c\}\}$ & $\{\{b\},\{c\},\{b, c\},\{a, b, c\}\}$ \\
\hline
\end{tabular}

Filter chain \# 30 and its STRT spectrum

\begin{tabular}{|l|l|l|l|l|}
\hline F1 & $\begin{array}{l}\{\{a\},\{b\},\{c\},\{a, b\},\{a, c\}, \\
\{b, c\},\{a, b, c\}\}\end{array}$ & $\begin{array}{l}\{\{a\},\{b\},\{c\},\{a, b\},\{a, c\}, \\
\{b, c\},\{a, b, c\}\}\end{array}$ & $\begin{array}{l}\{\{a\},\{b\},\{c\},\{a, b\},\{a, c\}, \\
\{b, c\},\{a, b, c\}\}\end{array}$ & $\begin{array}{l}\{\{a\},\{b\},\{c\},\{a, b\},\{a, c\}, \\
\{b, c\},\{a, b, c\}\}\end{array}$ \\
\hline F3 & $\begin{array}{l}\{\{a\},\{c\},\{a, b\},\{a, c\}, \\
\{b, c\},\{a, b, c\}\}\end{array}$ & $\begin{array}{l}\{\{a\},\{c\},\{a, b\},\{a, c\}, \\
\{b, c\},\{a, b, c\}\}\end{array}$ & $\begin{array}{l}\{\{a\},\{c\},\{a, b\},\{a, c\},\{b, c\}, \\
\{a, b, c\}\}\end{array}$ & $\{\{b\}\}$ \\
\hline F7 & $\begin{array}{l}\{\{c\},\{a, b\},\{a, c\},\{b, c\}, \\
\{a, b, c\}\}\end{array}$ & $\begin{array}{l}\{\{c\},\{a, b\},\{a, c\},\{b, c\}, \\
\{a, b, c\}\}\end{array}$ & $\{\{a\},\{b\}\}$ & $\{\{a\},\{b\},\{c\}\}$ \\
\hline F11 & $\{\{a, b\},\{a, c\},\{b, c\},\{a, b, c\}\}$ & $\{\{a, b\},\{a, c\},\{b, c\},\{a, b, c\}\}$ & $\{\{a\},\{c\}\}$ & $\{\{b\},\{c\}\}$ \\
\hline F14 & $\{\{a, c\},\{b, c\},\{a, b, c\}\}$ & $\{\{a\},\{b\},\{c\},\{a, b\}\}$ & $\begin{array}{l}\{\{a\},\{b\},\{c\},\{a, b\},\{a, c\}, \\
\{b, c\}\}\end{array}$ & $\begin{array}{l}\{a a\},\{b\},\{c\},\{a, b\},\{a, c\}, \\
\{b, c\},\{a, b, c\}\}\end{array}$ \\
\hline F17 & $\{\{b, c\},\{a, b, c\}\}$ & $\{\{a\},\{c\},\{a, b\},\{a, c\}\}$ & $\begin{array}{l}\{\{a\},\{c\},\{a, b\},\{a, c\},\{b, c\}, \\
\{a, b, c\}\}\end{array}$ & $\{\{b\},\{a, b, c\}\}$ \\
\hline F18 & $\{\{a, b, c\}\}$ & $\{\{c\},\{a, b\},\{a, c\},\{b, c\}\}$ & $\{a a\},\{b\},\{a, c\},\{b, c\}\}$ & $\begin{array}{l}\{\{a\},\{b\},\{c\},\{a, c\},\{b, c\}, \\
\{a, b, c\}\}\end{array}$ \\
\hline- & $\{\Phi\}$ & $\{\{a, b\},\{a, c\},\{b, c\},\{a, b, c\}\}$ & $\{\{a\},\{c\},\{b, c\},\{a, b, c\}\}$ & $\{\{b\},\{c\},\{a, c\},\{a, b, c\}\}$ \\
\hline
\end{tabular}

Filter chain \# 31 and its STRT spectrum

\begin{tabular}{|l|l|l|l|l|}
\hline F1 & $\begin{array}{l}\{\{a\},\{b\},\{c\},\{a, b\},\{a, c\}, \\
\{b, c\},\{a, b, c\}\}\end{array}$ & $\begin{array}{l}\{\{a\},\{b\},\{c\},\{a, b\},\{a, c\}, \\
\{b, c\},\{a, b, c\}\}\end{array}$ & $\begin{array}{l}\{\{a\},\{b\},\{c\},\{a, b\},\{a, c\}, \\
\{b, c\},\{a, b, c\}\}\end{array}$ & $\begin{array}{l}\{\{a\},\{b\},\{c\},\{a, b\},\{a, c\}, \\
\{b, c\},\{a, b, c\}\}\end{array}$ \\
\hline F3 & $\begin{array}{l}\{\{a\},\{c\},\{a, b\},\{a, c\}, \\
\{b, c\},\{a, b, c\}\}\end{array}$ & $\begin{array}{l}\{\{a\},\{c\},\{a, b\},\{a, c\}, \\
\{b, c\},\{a, b, c\}\}\end{array}$ & $\begin{array}{l}\{\{a\},\{c\},\{a, b\},\{a, c\}, \\
\{b, c\},\{a, b, c\}\}\end{array}$ & $\{\{b\}\}$ \\
\hline F7 & $\begin{array}{l}\{\{c\},\{a, b\},\{a, c\},\{b, c\}, \\
\{a, b, c\}\}\end{array}$ & $\begin{array}{l}\{\{c\},\{a, b\},\{a, c\},\{b, c\}, \\
\{a, b, c\}\}\end{array}$ & $\{\{a\},\{b\}\}$ & $\{\{a\},\{b\},\{a, b\}\}$ \\
\hline$F 10$ & $\{\{c\},\{a, c\},\{b, c\},\{a, b, c\}\}$ & $\{\{c\},\{a, c\},\{b, c\},\{a, b, c\}\}$ & $\{\{a\},\{a, b\}\}$ & $\{\{b\},\{a, b\}\}$ \\
\hline F14 & $\{\{a, c\},\{b, c\},\{a, b, c\}\}$ & $\{\{a\},\{b\},\{c\},\{a, b\}\}$ & $\begin{array}{l}\{\{a\},\{b\},\{c\},\{a, b\},\{a, c\}, \\
\{b, c\}\}\end{array}$ & $\begin{array}{l}\{\{a\},\{b\},\{c\},\{a, b\},\{a, c\}, \\
\{b, c\}, \\
\{a, b, c\}\}\end{array}$ \\
\hline F16 & $\{\{a, c\},\{a, b, c\}\}$ & $\{\{a\},\{c\},\{a, b\},\{b, c\}\}$ & $\begin{array}{l}\{\{a\},\{c\},\{a, b\},\{a, c\},\{b, c\}, \\
\{a, b, c\}\}\end{array}$ & $\{\{b\},\{a, b, c\}\}$ \\
\hline F18 & $\{\{a, b, c\}\}$ & $\{\{c\},\{a, b\},\{a, c\},\{b, c\}\}$ & $\{\{a\},\{b\},\{a, c\},\{b, c\}\}$ & $\begin{array}{l}\{\{a\},\{b\},\{a, b\},\{a, c\},\{b, c\}, \\
\{a, b, c\}\}\end{array}$ \\
\hline- & $\{\Phi\}$ & $\{\{c\},\{a, c\},\{b, c\},\{a, b, c\}\}$ & $\{\{a\},\{a, b\},\{a, c\},\{a, b, c\}\}$ & $\{\{b\},\{a, b\},\{b, c\},\{a, b, c\}\}$ \\
\hline
\end{tabular}

Filter chain \# 32 and its STRT spectrum

\begin{tabular}{|c|c|c|c|c|}
\hline $\mathrm{F} 1$ & $\begin{array}{l}\{\{a\},\{b\},\{c\},\{a, b\},\{a \\
c\} \\
\{b, c\},\{a, b, c\}\}\end{array}$ & $\begin{array}{l}\{\{a\},\{b\},\{c\},\{a, b\},\{a, c\} \\
\{b, c\},\{a, b, c\}\}\end{array}$ & $\begin{array}{l}\{a \mathrm{a}\},\{b\},\{c\},\{a, b\},\{a, c\} \\
\{b, c\},\{a, b, c\}\}\end{array}$ & $\begin{array}{l}\{\{a\},\{b\},\{c\},\{a, b\},\{a, c \\
\}, \\
\{b, c\},\{a, b, c\}\}\end{array}$ \\
\hline F3 & $\begin{array}{l}\{\{a\},\{c\},\{a, b\},\{a, c\} \\
\{b, c\},\{a, b, c\}\}\end{array}$ & $\begin{array}{l}\{a a\},\{c\},\{a, b\},\{a, c\},\{b, c\} \\
\{a, b, c\}\}\end{array}$ & $\begin{array}{l}\{\{a\},\{c\},\{a, b\},\{a, c\},\{b, c\} \\
\{a, b, c\}\}\end{array}$ & $\{\{b\}\}$ \\
\hline F7 & $\begin{array}{l}\{\{c\},\{a, b\},\{a, c\},\{b, c\}, \\
\{a, b, c\}\}\end{array}$ & $\begin{array}{l}\{\{c\},\{a, b\},\{a, c\},\{b, c\} \\
\{a, b, c\}\}\end{array}$ & $\{\{a\},\{b\}\}$ & $\{\{a\},\{b\},\{a, b\}\}$ \\
\hline F10 & $\begin{array}{l}\{\{c\},\{a, c\},\{b, c\},\{a, b, c \\
\}\}\end{array}$ & $\{\{c\},\{a, c\},\{b, c\},\{a, b, c\}\}$ & $\{\{a\},\{a, b\}\}$ & $\{\{b\},\{a, b\}\}$ \\
\hline F14 & $\{\{a, c\},\{b, c\},\{a, b, c\}\}$ & $\{\{a\},\{b\},\{c\},\{a, b\}\}$ & $\begin{array}{l}\{a\},\{b\},\{c\},\{a, b\},\{a, c\},\{b, c\} \\
\}\end{array}$ & $\begin{array}{l}\{\{a\},\{b\},\{c\},\{a, b\},\{a, c \\
\}, \\
\{b, c\},\{a, b, c\}\}\end{array}$ \\
\hline F17 & $\{\{b, c\},\{a, b, c\}\}$ & $\{\{a\},\{c\},\{a, b\},\{a, c\}\}$ & $\begin{array}{l}\{\{a\},\{c\},\{a, b\},\{a, c\},\{b, c\} \\
\{a, b, c\}\}\end{array}$ & $\{\{b\},\{a, b, c\}\}$ \\
\hline F18 & $\{\{a, b, c\}\}$ & $\{\{c\},\{a, b\},\{a, c\},\{b, c\}\}$ & $\{\{a\},\{b\},\{a, c\},\{b, c\}\}$ & $\begin{array}{l}\{\{a\},\{b\},\{a, b\},\{a, c\},\{b \\
, c\},\end{array}$ \\
\hline
\end{tabular}




\begin{tabular}{|l|l|l|l|l|}
\hline & & & & $\{a, b, c\}\}$ \\
\hline- & $\{\Phi\}$ & $\{\{c\},\{a, c\},\{b, c\},\{a, b, c\}\}$ & $\{\{a\},\{a, b\},\{b, c\},\{a, b, c\}\}$ & $\begin{array}{l}\{\{b\},\{a, b\},\{a, c\},\{a, b, c \\
\}\}\end{array}$ \\
\hline
\end{tabular}

Filter chain \# 33 and its STRT spectrum

\begin{tabular}{|c|c|c|c|c|}
\hline F1 & $\begin{array}{l}\{\{a\},\{b\},\{c\},\{a, b\},\{a, c \\
\}, \\
\{b, c\},\{a, b, c\}\}\end{array}$ & $\begin{array}{l}\{\{a\},\{b\},\{c\},\{a, b\},\{a, c\}, \\
\{b, c\},\{a, b, c\}\}\end{array}$ & $\begin{array}{l}\{\{a\},\{b\},\{c\},\{a, b\},\{a, c\} \\
\{b, c\},\{a, b, c\}\}\end{array}$ & $\begin{array}{l}\{\{a\},\{b\},\{c\},\{a, b\},\{ \\
a, c\}, \\
\{b, c\},\{a, b, c\}\}\end{array}$ \\
\hline F4 & $\begin{array}{l}\{\{b\},\{c\},\{a, b\},\{a, c\} \\
\{b, c\},\{a, b, c\}\}\end{array}$ & $\begin{array}{l}\{\{b\},\{c\},\{a, b\},\{a, c\} \\
\{b, c\},\{a, b, c\}\}\end{array}$ & $\begin{array}{l}\{\{b\},\{c\},\{a, b\},\{a, c\},\{b, c\},\{a, b \\
, c\}\}\end{array}$ & $\{\{a\}\}$ \\
\hline F6 & $\begin{array}{l}\{\{b\},\{a, b\},\{a, c\},\{b, c\}, \\
\{a, b, c\}\}\end{array}$ & $\begin{array}{l}\{\{b\},\{a, b\},\{a, c\},\{b, c\} \\
\{a, b, c\}\}\end{array}$ & $\{\{a\},\{c\}\}$ & $\{\{a\},\{b\},\{c\}\}$ \\
\hline F11 & $\begin{array}{l}\{\{a, b\},\{a, c\},\{b, c\},\{a, b, \\
c\}\}\end{array}$ & $\begin{array}{l}\{\{a, b\},\{a, c\},\{b, c\},\{a, b, c \\
\}\}\end{array}$ & $\{\{b\},\{c\}\}$ & $\{\{a\},\{b\}\}$ \\
\hline F12 & $\{\{a, b\},\{a, c\},\{a, b, c\}\}$ & $\{\{a\},\{b\},\{c\},\{b, c\}\}$ & $\{\{a\},\{b\},\{c\},\{a, b\},\{a, c\},\{b, c\}\}$ & $\begin{array}{l}\{\{a\},\{b\},\{c\},\{a, b\} \\
\{a, c\},\{b, c\},\{a, b, c\}\}\end{array}$ \\
\hline F15 & $\{\{a, b\},\{a, b, c\}\}$ & $\{\{b\},\{c\},\{a, c\},\{b, c\}\}$ & $\begin{array}{l}\{\{b\},\{c\},\{a, b\},\{a, c\},\{b, c\}, \\
\{a, b, c\}\}\end{array}$ & $\{\{a\},\{a, b, c\}\}$ \\
\hline F18 & $\{\{a, b, c\}\}$ & $\{\{b\},\{a, b\},\{a, c\},\{b, c\}\}$ & $\{\{a\},\{c\},\{a, b\},\{a, c\}\}$ & $\begin{array}{l}\{\{a\},\{b\},\{c\},\{a, b\} \\
\{a, c\},\{a, b, c\}\}\end{array}$ \\
\hline - & $\{\Phi\}$ & $\{\{a, b\},\{a, c\},\{b, c\},\{a, b, c$ & $\{\{b\},\{c\},\{a, b\},\{a, b, c\}\}$ & $\begin{array}{l}\{\{a\},\{b\},\{a, c\},\{a, b, c \\
\}\}\end{array}$ \\
\hline
\end{tabular}

Filter chain \# 34 and its STRT spectrum

\begin{tabular}{|c|c|c|c|c|}
\hline $\mathrm{F} 1$ & $\begin{array}{l}\{\{a\},\{b\},\{c\},\{a, b\},\{a, c\} \\
\{b, c\},\{a, b, c\}\}\end{array}$ & $\begin{array}{l}\{\{a\},\{b\},\{c\},\{a, b\},\{a, c\} \\
\{b, c\},\{a, b, c\}\}\end{array}$ & $\begin{array}{l}\{\{a\},\{b\},\{c\},\{a, b\},\{a, c\} \\
\{b, c\},\{a, b, c\}\}\end{array}$ & $\begin{array}{l}\{a \mathrm{a}\},\{b\},\{c\},\{a, b\}, \\
\{a, c\}, \\
\{b, c\},\{a, b, c\}\}\end{array}$ \\
\hline F4 & $\begin{array}{l}\{\{b\},\{c\},\{a, b\},\{a, c\} \\
\{b, c\},\{a, b, c\}\}\end{array}$ & $\begin{array}{l}\{\{b\},\{c\},\{a, b\},\{a, c\},\{b, c\} \\
\{a, b, c\}\}\end{array}$ & $\begin{array}{l}\{\{b\},\{c\},\{a, b\},\{a, c\} \\
\{b, c\},\{a, b, c\}\}\end{array}$ & $\{\{a\}\}$ \\
\hline F6 & $\begin{array}{l}\{\{b\},\{a, b\},\{a, c\},\{b, c\}, \\
\{a, b, c\}\}\end{array}$ & $\begin{array}{l}\{\{b\},\{a, b\},\{a, c\},\{b, c\} \\
\{a, b, c\}\}\end{array}$ & $\{\{a\},\{c\}\}$ & $\{\{a\},\{b\},\{c\}\}$ \\
\hline $\begin{array}{l}\mathrm{F} 1 \\
1\end{array}$ & $\begin{array}{l}\{\{a, b\},\{a, c\},\{b, c\},\{a, b, c\} \\
\}\end{array}$ & $\begin{array}{l}\{\{a, b\},\{a, c\},\{b, c\},\{a, b, c\} \\
\}\end{array}$ & $\{\{b\},\{c\}\}$ & $\{\{a\},\{b\}\}$ \\
\hline $\begin{array}{l}\text { F1 } \\
2\end{array}$ & $\{\{a, b\},\{a, c\},\{a, b, c\}\}$ & $\{\{a\},\{b\},\{c\},\{b, c\}\}$ & $\begin{array}{l}\{\{a\},\{b\},\{c\},\{a, b\},\{a, c\},\{b, c\} \\
\}\end{array}$ & $\begin{array}{l}\{\{a\},\{b\},\{c\},\{a, b\}, \\
\{a, c\} \\
\{b, c\},\{a, b, c\}\}\end{array}$ \\
\hline $\begin{array}{l}\mathrm{F} 1 \\
6\end{array}$ & $\{\{a, c\},\{a, b, c\}\}$ & $\{\{b\},\{c\},\{a, b\},\{b, c\}\}$ & $\begin{array}{l}\{\{b\},\{c\},\{a, b\},\{a, c\},\{b, c\}, \\
\{a, b, c\}\}\end{array}$ & $\{\{a\},\{a, b, c\}\}$ \\
\hline $\begin{array}{l}\text { F1 } \\
8\end{array}$ & $\{\{a, b, c\}\}$ & $\{\{b\},\{a, b\},\{a, c\},\{b, c\}\}$ & $\{\{a\},\{c\},\{a, b\},\{a, c\}\}$ & $\begin{array}{l}\{\{a\},\{b\},\{c\},\{a, b\}, \\
\{a, c\},\{a, b, c\}\}\end{array}$ \\
\hline - & $\{\Phi\}$ & $\begin{array}{l}\{\{a, b\},\{a, c\},\{b, c\},\{a, b, c\} \\
\}\end{array}$ & $\{\{b\},\{c\},\{a, c\},\{a, b, c\}\}$ & $\begin{array}{l}\{\{a\},\{b\},\{a, b\},\{a, b \\
, c\}\}\end{array}$ \\
\hline
\end{tabular}

Filter chain \# 35 and its STRT spectrum

\begin{tabular}{|l|l|l|l|l|}
\hline F1 & $\begin{array}{l}\{\{a\},\{b\},\{c\},\{a, b\},\{a, c\} \\
,\{b, c\},\{a, b, c\}\}\end{array}$ & $\begin{array}{l}\{\{a\},\{b\},\{c\},\{a, b\},\{a, c\}, \\
\{b, c\},\{a, b, c\}\}\end{array}$ & $\begin{array}{l}\{\{a\},\{b\},\{c\},\{a, b\},\{a, c\}, \\
\{b, c\},\{a, b, c\}\}\end{array}$ & $\begin{array}{l}\{\{a\},\{b\},\{c\},\{a, b\},\{a, c\}, \\
\{b, c\},\{a, b, c\}\}\end{array}$ \\
\hline F4 & $\begin{array}{l}\{\{b\},\{c\},\{a, b\},\{a, c\}, \\
\{b, c\},\{a, b, c\}\}\end{array}$ & $\begin{array}{l}\{\{b\},\{c\},\{a, b\},\{a, c\}, \\
\{b, c\},\{a, b, c\}\}\end{array}$ & $\begin{array}{l}\{\{b\},\{c\},\{a, b\},\{a, c\}, \\
\{b, c\},\{a, b, c\}\}\end{array}$ & $\{\{a\}\}$ \\
\hline F6 & $\begin{array}{l}\{\{b\},\{a, b\},\{a, c\},\{b, c\}, \\
\{a, b, c\}\}\end{array}$ & $\begin{array}{l}\{\{b\},\{a, b\},\{a, c\},\{b, c\}, \\
\{a, b, c\}\}\end{array}$ & $\{\{a\},\{c\}\}$ & $\{\{a\},\{b\},\{c\}\}$ \\
\hline F11 & $\begin{array}{l}\{\{a, b\},\{a, c\},\{b, c\}, \\
\{a, b, c\}\}\end{array}$ & $\begin{array}{l}\{\{a, b\},\{a, c\},\{b, c\}, \\
\{a, b, c\}\}\end{array}$ & $\{\{b\},\{c\}\}$ & $\{\{a\},\{b\}\}$ \\
\hline F13 & $\{\{a, b\},\{b, c\},\{a, b, c\}\}$ & $\{\{a\},\{b\},\{c\},\{a, c\}\}$ & $\begin{array}{l}\{\{a\},\{b\},\{c\},\{a, b\},\{a, c\}, \\
\{b, c\}\}\end{array}$ & $\begin{array}{l}\{\{a\},\{b\},\{c\},\{a, b\},\{a, c\}, \\
\{b, c\},\{a, b, c\}\}\end{array}$ \\
\hline F15 & $\{\{a, b\},\{a, b, c\}\}$ & $\{\{b\},\{c\},\{a, c\},\{b, c\}\}$ & $\begin{array}{l}\{\{b\},\{c\},\{a, b\},\{a, c\}, \\
\{b, c\},\{a, b, c\}\}\end{array}$ & $\{\{a\},\{a, b, c\}\}$ \\
\hline F18 & $\{\{a, b, c\}\}$ & $\{\{b\},\{a, b\},\{a, c\},\{b, c\}\}$ & $\{\{a\},\{c\},\{a, b\},\{b, c\}\}$ & $\{\{a\},\{b\},\{c\},\{a, b\},\{b, c\}$, \\
\hline
\end{tabular}




\begin{tabular}{|l|l|l|l|l|}
\hline & & & & $\{a, b, c\}\}$ \\
\hline- & $\{\Phi\}$ & $\begin{array}{l}\{\{a, b\},\{a, c\},\{b, c\}, \\
\{a, b, c\}\}\end{array}$ & $\{\{b\},\{c\},\{a, b\},\{a, b, c\}\}$ & $\{\{a\},\{b\},\{b, c\},\{a, b, c\}\}$ \\
\hline
\end{tabular}

Filter chain \# 36 and its STRT spectrum

\begin{tabular}{|c|c|c|c|c|}
\hline F1 & $\begin{array}{l}\{a \mathrm{a}\},\{b\},\{c\},\{a, b\},\{a, c\} \\
\{b, c\},\{a, b, c\}\}\end{array}$ & $\begin{array}{l}\{\{a\},\{b\},\{c\},\{a, b\},\{a, c\} \\
\{b, c\},\{a, b, c\}\}\end{array}$ & $\begin{array}{l}\{\{a\},\{b\},\{c\},\{a, b\},\{a, c\} \\
\{b, c\},\{a, b, c\}\}\end{array}$ & $\begin{array}{l}\{\{a\},\{b\},\{c\},\{a, b\},\{a, c\}, \\
\{b, c\},\{a, b, c\}\}\end{array}$ \\
\hline F4 & $\begin{array}{l}\{\{b\},\{c\},\{a, b\},\{a, c\} \\
\{b, c\},\{a, b, c\}\}\end{array}$ & $\begin{array}{l}\{\{b\},\{c\},\{a, b\},\{a, c\} \\
\{b, c\},\{a, b, c\}\}\end{array}$ & $\begin{array}{l}\{\{b\},\{c\},\{a, b\},\{a, c\} \\
\{b, c\},\{a, b, c\}\}\end{array}$ & ( \\
\hline F6 & $\begin{array}{l}\{\{b\},\{a, b\},\{a, c\},\{b, c\} \\
\{a, b, c\}\}\end{array}$ & $\begin{array}{l}\{\{b\},\{a, b\},\{a, c\},\{b, c\} \\
\{a, b, c\}\}\end{array}$ & $\{\{a\},\{c\}\}$ & $\{\{a\},\{b\},\{c\}\}$ \\
\hline F11 & $\{\{a, b\},\{a, c\},\{b, c\},\{a, b, c\}\}$ & $\{\{a, b\},\{a, c\},\{b, c\},\{a, b, c\}\}$ & $\{\{b\},\{c\}\}$ & $\{\{a\},\{b\}\}$ \\
\hline $\mathrm{F} 13$ & $\{\{a, b\},\{b, c\},\{a, b, c\}\}$ & $\{\{a\},\{b\},\{c\},\{a, c\}\}$ & $\begin{array}{l}\{\{a\},\{b\},\{c\},\{a, b\},\{a, c\}, \\
\{b, c\}\}\end{array}$ & $\begin{array}{l}\{\{a\},\{b\},\{c\},\{a, b\},\{a, c\}, \\
\{b, c\},\{a, b, c\}\}\end{array}$ \\
\hline F17 & $\{\{b, c\},\{a, b, c\}\}$ & $\{\{b\},\{c\},\{a, b\},\{a, c\}\}$ & $\begin{array}{l}\{\{b\},\{c\},\{a, b\},\{a, c\} \\
\{b, c\},\{a, b, c\}\}\end{array}$ & $\{\{a\},\{a, b, c\}\}$ \\
\hline F18 & $\{\{a, b, c\}\}$ & $\{\{b\},\{a, b\},\{a, c\},\{b, c\}\}$ & $\{\{a\},\{c\},\{a, b\},\{b, c\}\}$ & $\begin{array}{l}\{a a\},\{b\},\{c\},\{a, b\},\{b, c\}, \\
\{a, b, c\}\}\end{array}$ \\
\hline- & $\{\Phi\}$ & $\{\{a, b\},\{a, c\},\{b, c\},\{a, b, c\}\}$ & $\{\{b\},\{c\},\{b, c\},\{a, b, c\}\}$ & $\{\{a\},\{b\},\{a, b\},\{a, b, c\}\}$ \\
\hline
\end{tabular}

Filter chain \# 37 and its STRT spectrum

\begin{tabular}{|l|l|l|l|l|}
\hline F1 & $\begin{array}{l}\{\{a\},\{b\},\{c\},\{a, b\},\{a, c\} \\
\{b, c\},\{a, b, c\}\}\end{array}$ & $\begin{array}{l}\{\{a\},\{b\},\{c\},\{a, b\},\{a, c\}, \\
\{b, c\},\{a, b, c\}\}\end{array}$ & $\begin{array}{l}\{\{a\},\{b\},\{c\},\{a, b\},\{a, c\}, \\
\{b, c\},\{a, b, c\}\}\end{array}$ & $\begin{array}{l}\{\{a\},\{b\},\{c\},\{a, b\}, \\
\{a, c\},\{b, c\},\{a, b, c\}\}\end{array}$ \\
\hline F4 & $\begin{array}{l}\{\{b\},\{c\},\{a, b\},\{a, c\}, \\
\{b, c\},\{a, b, c\}\}\end{array}$ & $\begin{array}{l}\{\{b\},\{c\},\{a, b\},\{a, c\}, \\
\{b, c\},\{a, b, c\}\}\end{array}$ & $\begin{array}{l}\{\{b\},\{c\},\{a, b\},\{a, c\},\{b, c\}, \\
\{a, b, c\}\}\end{array}$ & $\{\{a\}\}$ \\
\hline$F 6$ & $\begin{array}{l}\{\{b\},\{a, b\},\{a, c\},\{b, c\}, \\
\{a, b, c\}\}\end{array}$ & $\begin{array}{l}\{\{b\},\{a, b\},\{a, c\},\{b, c\}, \\
\{a, b, c\}\}\end{array}$ & $\{\{a\},\{c\}\}$ & $\{\{a\},\{b\},\{c\}\}$ \\
\hline$F 11$ & $\begin{array}{l}\{\{a, b\},\{a, c\},\{b, c\},\{a, b, \\
c\}\}\end{array}$ & $\begin{array}{l}\{\{a, b\},\{a, c\},\{b, c\},\{a, b, c \\
\}\}\end{array}$ & $\{\{b\},\{c\}\}$ & $\{\{a\},\{b\}\}$ \\
\hline$F 14$ & $\{\{a, c\},\{b, c\},\{a, b, c\}\}$ & $\{\{a\},\{b\},\{c\},\{a, b\}\}$ & $\begin{array}{l}\{\{a\},\{b\},\{c\},\{a, b\},\{a, c\}, \\
\{b, c\}\}\end{array}$ & $\begin{array}{l}\{a a\},\{b\},\{c\},\{a, b\}, \\
\{a, c\},\{b, c\},\{a, b, c\}\}\end{array}$ \\
\hline F16 & $\{\{a, c\},\{a, b, c\}\}$ & $\{\{b\},\{c\},\{a, b\},\{b, c\}\}$ & $\begin{array}{l}\{\{b\},\{c\},\{a, b\},\{a, c\},\{b, c\}, \\
\{a, b, c\}\}\end{array}$ & $\{\{a\},\{a, b, c\}\}$ \\
\hline F18 & $\{\{a, b, c\}\}$ & $\{\{b\},\{a, b\},\{a, c\},\{b, c\}\}$ & $\{\{a\},\{c\},\{a, c\},\{b, c\}\}$ & $\begin{array}{l}\{a b\},\{b\},\{c\},\{a, c\},\{b, c\} \\
\{a, b, c\}\}\end{array}$ \\
\hline- & $\{\Phi\}$ & $\begin{array}{l}\{\{a, b\},\{a, c\},\{b, c\},\{a, b, c \\
\}\}\end{array}$ & $\{\{b\},\{c\},\{a, c\},\{a, b, c\}\}$ & $\begin{array}{l}\{\{a,,\{b\},\{b, c\}, \\
\{a, b, c\}\}\end{array}$ \\
\hline
\end{tabular}

Filter chain \# 38 and its STRT spectrum

\begin{tabular}{|c|c|c|c|c|}
\hline F1 & $\begin{array}{l}\{\{a\},\{b\},\{c\},\{a, b\},\{a, c\} \\
\{b, c\},\{a, b, c\}\}\end{array}$ & $\begin{array}{l}\{\{a\},\{b\},\{c\},\{a, b\},\{a, c\} \\
\{b, c\},\{a, b, c\}\}\end{array}$ & $\begin{array}{l}\{\{a\},\{b\},\{c\},\{a, b\},\{a, c\} \\
\{b, c\},\{a, b, c\}\}\end{array}$ & $\begin{array}{l}\{\{a\},\{b\},\{c\},\{a, b\},\{a, c\}, \\
\{b, c\},\{a, b, c\}\}\end{array}$ \\
\hline $\mathrm{F} 4$ & $\begin{array}{l}\{\{b\},\{c\},\{a, b\},\{a, c\} \\
\{b, c\},\{a, b, c\}\}\end{array}$ & $\begin{array}{l}\{\{b\},\{c\},\{a, b\},\{a, c\},\{b, c\}, \\
\{a, b, c\}\}\end{array}$ & $\begin{array}{l}\{\{b\},\{c\},\{a, b\},\{a, c\},\{b, c\}, \\
\{a, b, c\}\}\end{array}$ & $\{\{a\}\}$ \\
\hline F6 & $\begin{array}{l}\{\{b\},\{a, b\},\{a, c\},\{b, c\}, \\
\{a, b, c\}\}\end{array}$ & $\begin{array}{l}\{\{b\},\{a, b\},\{a, c\},\{b, c\} \\
\{a, b, c\}\}\end{array}$ & $\{\{a\},\{c\}\}$ & $\{\{a\},\{b\},\{c\}\}$ \\
\hline F11 & $\begin{array}{l}\{\{a, b\},\{a, c\},\{b, c\} \\
\{a, b, c\}\}\end{array}$ & $\{\{a, b\},\{a, c\},\{b, c\},\{a, b, c\}\}$ & $\{\{b\},\{c\}\}$ & $\{\{a\},\{b\}\}$ \\
\hline F14 & $\{\{a, c\},\{b, c\},\{a, b, c\}\}$ & $\{\{a\},\{b\},\{c\},\{a, b\}\}$ & $\begin{array}{l}\{\{a\},\{b\},\{c\},\{a, b\} \\
\{a, c\},\{b, c\}\}\end{array}$ & $\begin{array}{l}\{\{a\},\{b\},\{c\},\{a, b\},\{a, c\}, \\
\{b, c\},\{a, b, c\}\}\end{array}$ \\
\hline $\mathrm{F} 17$ & $\{\{b, c\},\{a, b, c\}\}$ & $\{\{b\},\{c\},\{a, b\},\{a, c\}\}$ & $\begin{array}{l}\{\{b\},\{c\},\{a, b\},\{a, c\} \\
\{b, c\},\{a, b, c\}\}\end{array}$ & $\{\{a\},\{a, b, c\}\}$ \\
\hline F18 & $\{\{a, b, c\}\}$ & $\{\{b\},\{a, b\},\{a, c\},\{b, c\}\}$ & $\{\{a\},\{c\},\{a, c\},\{b, c\}\}$ & $\begin{array}{l}\{\{a\},\{b\},\{c\},\{a, c\},\{b, c\}, \\
\{a, b, c\}\}\end{array}$ \\
\hline- & $\{\Phi\}$ & $\{\{a, b\},\{a, c\},\{b, c\},\{a, b, c\}\}$ & $\{\{b\},\{c\},\{b, c\},\{a, b, c\}\}$ & $\{\{a\},\{b\},\{a, c\},\{a, b, c\}\}$ \\
\hline
\end{tabular}


Filter chain \# 39 and its STRT spectrum

\begin{tabular}{|l|l|l|l|l|}
\hline F1 & $\begin{array}{l}\{\{a\},\{b\},\{c\},\{a, b\},\{a, c\}, \\
\{b, c\},\{a, b, c\}\}\end{array}$ & $\begin{array}{l}\{\{a\},\{b\},\{c\},\{a, b\},\{a, c\}, \\
\{b, c\},\{a, b, c\}\}\end{array}$ & $\begin{array}{l}\{a b,\{b\},\{c\},\{a, b\},\{a, c\}, \\
\{b, c\},\{a, b, c\}\}\end{array}$ & $\begin{array}{l}\{\{a\},\{b\},\{c\},\{a, b\},\{a, c\}, \\
\{b, c\},\{a, b, c\}\}\end{array}$ \\
\hline F4 & $\begin{array}{l}\{\{b\},\{c\},\{a, b\},\{a, c\}, \\
\{b, c\},\{a, b, c\}\}\end{array}$ & $\begin{array}{l}\{\{b\},\{c\},\{a, b\},\{a, c\}, \\
\{b, c\},\{a, b, c\}\}\end{array}$ & $\begin{array}{l}\{\{b\},\{c\},\{a, b\},\{a, c\}, \\
\{b, c\},\{a, b, c\}\}\end{array}$ & $\{\{a\}\}$ \\
\hline F6 & $\begin{array}{l}\{\{b\},\{a, b\},\{a, c\},\{b, c\}, \\
\{a, b, c\}\}\end{array}$ & $\begin{array}{l}\{\{b\},\{a, b\},\{a, c\},\{b, c\}, \\
\{a, b, c\}\}\end{array}$ & $\{\{a\},\{c\}\}$ & $\{\{a\},\{c\},\{a, c\}\}$ \\
\hline F9 & $\{\{b\},\{a, b\},\{b, c\},\{a, b, c\}\}$ & $\{\{b\},\{a, b\},\{b, c\},\{a, b, c\}\}$ & $\{\{c\},\{a, c\}\}$ & $\{\{a\},\{a, c\}\}$ \\
\hline F13 & $\{\{a, b\},\{b, c\},\{a, b, c\}\}$ & $\{\{a\},\{b\},\{c\},\{a, c\}\}$ & $\begin{array}{l}\{\{a\},\{b\},\{c\},\{a, b\}, \\
\{a, c\},\{b, c\}\}\end{array}$ & $\begin{array}{l}\{\{a\},\{b\},\{c\},\{a, b\},\{a, c\}, \\
\{b, c\},\{a, b, c\}\}\end{array}$ \\
\hline F15 & $\{\{a, b\},\{a, b, c\}\}$ & $\{\{b\},\{c\},\{a, c\},\{b, c\}\}$ & $\begin{array}{l}\{\{b\},\{c\},\{a, b\},\{a, c\}, \\
\{b, c\},\{a, b, c\}\}\end{array}$ & $\{\{a\},\{a, b, c\}\}$ \\
\hline F18 & $\{\{a, b, c\}\}$ & $\{\{b\},\{a, b\},\{a, c\},\{b, c\}\}$ & $\{\{a\},\{c\},\{a, b\},\{b, c\}\}$ & $\begin{array}{l}\{\{a\},\{c\},\{a, b\},\{a, c\},\{b, c\}, \\
\{a, b, c\}\}\end{array}$ \\
\hline- & $\{\Phi\}$ & $\{\{b\},\{a, b\},\{b, c\},\{a, b, c\}\}$ & $\{\{c\},\{a, b\},\{a, c\},\{a, b, c\}\}$ & $\{\{a\},\{a, c\},\{b, c\},\{a, b, c\}\}$ \\
\hline
\end{tabular}

Filter chain \#40 and its STRT spectrum

\begin{tabular}{|c|c|c|c|c|}
\hline F1 & $\begin{array}{l}\{\{a\},\{b\},\{c\},\{a, b\},\{a, c\}, \\
\{b, c\},\{a, b, c\}\}\end{array}$ & $\begin{array}{l}\{\{a\},\{b\},\{c\},\{a, b\},\{a, c\}, \\
\{b, c\},\{a, b, c\}\}\end{array}$ & $\begin{array}{l}\{\{a\},\{b\},\{c\},\{a, b\},\{a, c\}, \\
\{b, c\},\{a, b, c\}\}\end{array}$ & $\begin{array}{l}\{\{a\},\{b\},\{c\},\{a, b\},\{a, c\}, \\
\{b, c\},\{a, b, c\}\}\end{array}$ \\
\hline F4 & $\begin{array}{l}\{\{b\},\{c\},\{a, b\},\{a, c\} \\
\{b, c\},\{a, b, c\}\}\end{array}$ & $\begin{array}{l}\{\{b\},\{c\},\{a, b\},\{a, c\} \\
\{b, c\},\{a, b, c\}\}\end{array}$ & $\begin{array}{l}\{\{b\},\{c\},\{a, b\},\{a, c\} \\
\{b, c\},\{a, b, c\}\}\end{array}$ & $\{\{a\}\}$ \\
\hline F6 & $\begin{array}{l}\{\{b\},\{a, b\},\{a, c\},\{b, c\}, \\
\{a, b, c\}\}\end{array}$ & $\begin{array}{l}\{\{b\},\{a, b\},\{a, c\},\{b, c\}, \\
\{a, b, c\}\}\end{array}$ & $\{\{a\},\{c\}\}$ & $\{\{a\},\{c\},\{a, c\}\}$ \\
\hline F9 & $\{\{b\},\{a, b\},\{b, c\},\{a, b, c\}\}$ & $\{\{b\},\{a, b\},\{b, c\},\{a, b, c\}\}$ & $\{\{c\},\{a, c\}\}$ & $\{\{\mathrm{a}\},\{\mathrm{a}, \mathrm{c}\}\}$ \\
\hline F13 & $\{\{a, b\},\{b, c\},\{a, b, c\}\}$ & $\{\{a\},\{b\},\{c\},\{a, c\}\}$ & $\begin{array}{l}\{\{a\},\{b\},\{c\},\{a, b\}, \\
\{a, c\},\{b, c\}\}\end{array}$ & $\begin{array}{l}\{\{a\},\{b\},\{c\},\{a, b\},\{a, c\}, \\
\{b, c\},\{a, b, c\}\}\end{array}$ \\
\hline F17 & $\{\{b, c\},\{a, b, c\}\}$ & $\{\{b\},\{c\},\{a, b\},\{a, c\}\}$ & $\begin{array}{l}\{\{b\},\{c\},\{a, b\},\{a, c\}, \\
\{b, c\},\{a, b, c\}\}\end{array}$ & $\{\{a\},\{a, b, c\}\}$ \\
\hline F18 & $\{\{a, b, c\}\}$ & $\{\{b\},\{a, b\},\{a, c\},\{b, c\}\}$ & $\{\{a\},\{c\},\{a, b\},\{b, c\}\}$ & $\begin{array}{l}\{\{a\},\{c\},\{a, b\},\{a, c\}, \\
\{b, c\},\{a, b, c\}\}\end{array}$ \\
\hline - & $\{\Phi\}$ & $\{\{b\},\{a, b\},\{b, c\},\{a, b, c\}\}$ & $\begin{array}{l}\{\{c\},\{a, c\},\{b, c\}, \\
\{a, b, c\}\}\end{array}$ & $\{\{a\},\{a, b\},\{a, c\},\{a, b, c\}\}$ \\
\hline
\end{tabular}

Filter chain \# 41 and its STRT spectrum

\begin{tabular}{|l|l|l|l|l|}
\hline F1 & $\begin{array}{l}\{\{a\},\{b\},\{c\},\{a, b\},\{a, c\}, \\
\{b, c\},\{a, b, c\}\}\end{array}$ & $\begin{array}{l}\{\{a\},\{b\},\{c\},\{a, b\},\{a, c\}, \\
\{b, c\},\{a, b, c\}\}\end{array}$ & $\begin{array}{l}\{\{a\},\{b\},\{c\},\{a, b\},\{a, c\}, \\
\{b, c\},\{a, b, c\}\}\end{array}$ & $\begin{array}{l}\{a a\},\{b\},\{c\},\{a, b\},\{a, c\}, \\
\{b, c\},\{a, b, c\}\}\end{array}$ \\
\hline F4 & $\begin{array}{l}\{\{b\},\{c\},\{a, b\},\{a, c\}, \\
\{b, c\},\{a, b, c\}\}\end{array}$ & $\begin{array}{l}\{\{b\},\{c\},\{a, b\},\{a, c\}, \\
\{b, c\},\{a, b, c\}\}\end{array}$ & $\begin{array}{l}\{\{b\},\{c\},\{a, b\},\{a, c\}, \\
\{b, c\},\{a, b, c\}\}\end{array}$ & $\{\{a\}\}$ \\
\hline F7 & $\begin{array}{l}\{\{c\},\{a, b\},\{a, c\},\{b, c\}, \\
\{a, b, c\}\}\end{array}$ & $\begin{array}{l}\{\{c\},\{a, b\},\{a, c\},\{b, c\}, \\
\{a, b, c\}\}\end{array}$ & $\{\{a\},\{b\}\}$ & $\{\{a\},\{b\},\{c\}\}$ \\
\hline F11 & $\begin{array}{l}\{\{a, b\},\{a, c\},\{b, c\}, \\
\{a, b, c\}\}\end{array}$ & $\begin{array}{l}\{\{a, b\},\{a, c\},\{b, c\}, \\
\{a, b, c\}\}\end{array}$ & $\{\{b\},\{c\}\}$ & $\{\{a\},\{c\}\}$ \\
\hline F12 & $\{\{a, b\},\{a, c\},\{a, b, c\}\}$ & $\{\{a\},\{b\},\{c\},\{b, c\}\}$ & $\begin{array}{l}\{\{a\},\{b\},\{c\},\{a, b\},\{a, c\}, \\
\{b, c\}\}\end{array}$ & $\begin{array}{l}\{\{a\},\{b\},\{c\},\{a, b\},\{a, c\}, \\
\{b, c\},\{a, b, c\}\}\end{array}$ \\
\hline F15 & $\{\{a, b\},\{a, b, c\}\}$ & $\{\{b\},\{c\},\{a, c\},\{b, c\}\}$ & $\begin{array}{l}\{\{b\},\{c\},\{a, b\},\{a, c\}, \\
\{b, c\},\{a, b, c\}\}\end{array}$ & $\{\{a\},\{a, b, c\}\}$ \\
\hline F18 & $\{\{a, b, c\}\}$ & $\{\{c\},\{a, b\},\{a, c\},\{b, c\}\}$ & $\{\{a\},\{b\},\{a, b\},\{a, c\}\}$ & $\begin{array}{l}\{a a\},\{b\},\{c\},\{a, b\},\{a, c\}, \\
\{a, b, c\}\}\end{array}$ \\
\hline- & $\{\Phi\}$ & $\begin{array}{l}\{\{a, b\},\{a, c\},\{b, c\}, \\
\{a, b, c\}\}\end{array}$ & $\{\{b\},\{c\},\{a, b\},\{a, b, c\}\}$ & $\{\{a\},\{c\},\{a, c\},\{a, b, c\}\}$ \\
\hline
\end{tabular}

Filter chain \# 42 and its STRT spectrum

\begin{tabular}{|l|l|l|l|l|}
\hline F1 & $\begin{array}{l}\{\{a\},\{b\},\{c\},\{a, b\},\{a, c\}, \\
\\
\{b, c\},\{a, b, c\}\}\end{array}$ & $\begin{array}{l}\{\{a\},\{b\},\{c\},\{a, b\},\{a, c\}, \\
\{b, c\},\{a, b, c\}\}\end{array}$ & $\begin{array}{l}\{\{a\},\{b\},\{c\},\{a, b\},\{a, c\}, \\
\{b, c\},\{a, b, c\}\}\end{array}$ & $\begin{array}{l}\{\{a\},\{b\},\{c\},\{a, b\},\{a, c\}, \\
\{b, c\},\{a, b, c\}\}\end{array}$ \\
\hline F4 & $\begin{array}{l}\{\{b\},\{c\},\{a, b\},\{a, c\}, \\
\{b, c\},\{a, b, c\}\}\end{array}$ & $\begin{array}{l}\{\{b\},\{c\},\{a, b\},\{a, c\}, \\
\{b, c\},\{a, b, c\}\}\end{array}$ & $\begin{array}{l}\{\{b\},\{c\},\{a, b\},\{a, c\}, \\
\{b, c\},\{a, b, c\}\}\end{array}$ & $\{\{a\}\}$ \\
\hline F7 & $\begin{array}{l}\{\{c\},\{a, b\},\{a, c\},\{b, c\}, \\
\text { fa,b,c\}\} }\end{array}$ & $\begin{array}{l}\{\{c\},\{a, b\},\{a, c\},\{b, c\}, \\
\{a, b, c\}\}\end{array}$ & $\{\{a\},\{b\}\}$ & $\{\{a\},\{b\},\{c\}\}$ \\
\hline F11 & $\begin{array}{l}\{\{a, b\},\{a, c\},\{b, c\}, \\
\{a, b, c\}\}\end{array}$ & $\begin{array}{l}\{\{a, b\},\{a, c\},\{b, c\}, \\
\{a, b, c\}\}\end{array}$ & $\{\{b\},\{c\}\}$ & $\{a b\},\{c\}\}$ \\
\hline
\end{tabular}




\begin{tabular}{|l|l|l|l|l|}
\hline F12 & $\{\{a, b\},\{a, c\},\{a, b, c\}\}$ & $\{\{a\},\{b\},\{c\},\{b, c\}\}$ & $\begin{array}{l}\{\{a\},\{b\},\{c\},\{a, b\},\{a, c\}, \\
\{b, c\}\}\end{array}$ & $\begin{array}{l}\{\{a\},\{b\},\{c\},\{a, b\},\{a, c\}, \\
\{b, c\},\{a, b, c\}\}\end{array}$ \\
\hline F16 & $\{\{a, c\},\{a, b, c\}\}$ & $\{\{b\},\{c\},\{a, b\},\{b, c\}\}$ & $\begin{array}{l}\{\{b\},\{c\},\{a, b\},\{a, c\}, \\
\{b, c\},\{a, b, c\}\}\end{array}$ & $\{\{a\},\{a, b, c\}\}$ \\
\hline F18 & $\{\{a, b, c\}\}$ & $\{\{c\},\{a, b\},\{a, c\},\{b, c\}\}$ & $\{\{a\},\{b\},\{a, b\},\{a, c\}\}$ & $\begin{array}{l}\{\{a\},\{b\},\{c\},\{a, b\},\{a, c\}, \\
\{a, b, c\}\}\end{array}$ \\
\hline- & $\{\Phi\}$ & $\begin{array}{l}\{\{a, b\},\{a, c\},\{b, c\}, \\
\{a, b, c\}\}\end{array}$ & $\{\{b\},\{c\},\{a, c\},\{a, b, c\}\}$ & $\{\{a\},\{c\},\{a, b\},\{a, b, c\}\}$ \\
\hline
\end{tabular}

Filter chain \# 43 and its STRT spectrum

\begin{tabular}{|c|c|c|c|c|}
\hline F1 & $\begin{array}{l}\{a \mathrm{a}\},\{b\},\{c\},\{a, b\},\{a, c\} \\
\{b, c\},\{a, b, c\}\}\end{array}$ & $\begin{array}{l}\{a \mathrm{a}\},\{b\},\{c\},\{a, b\},\{a, c\} \\
\{b, c\},\{a, b, c\}\}\end{array}$ & $\begin{array}{l}\{\{a\},\{b\},\{c\},\{a, b\},\{a, c\} \\
\{b, c\},\{a, b, c\}\}\end{array}$ & $\begin{array}{l}\{a \mathrm{a}\},\{b\},\{c\},\{a, b\},\{a, c\}, \\
\{b, c\},\{a, b, c\}\}\end{array}$ \\
\hline F4 & $\begin{array}{l}\{\{b\},\{c\},\{a, b\},\{a, c\} \\
\{b, c\},\{a, b, c\}\}\end{array}$ & $\begin{array}{l}\{\{b\},\{c\},\{a, b\},\{a, c\} \\
\{b, c\},\{a, b, c\}\}\end{array}$ & $\begin{array}{l}\{\{b\},\{c\},\{a, b\},\{a, c\} \\
\{b, c\},\{a, b, c\}\}\end{array}$ & $\{\{a\}\}$ \\
\hline F7 & $\begin{array}{l}\{\{c\},\{a, b\},\{a, c\},\{b, c\}, \\
\{a, b, c\}\}\end{array}$ & $\begin{array}{l}\{\{c\},\{a, b\},\{a, c\},\{b, c\}, \\
\{a, b, c\}\}\end{array}$ & $\{\{a\},\{b\}\}$ & $\{\{a\},\{b\},\{c\}\}$ \\
\hline F11 & $\{\{a, b\},\{a, c\},\{b, c\},\{a, b, c\}\}$ & $\{\{a, b\},\{a, c\},\{b, c\},\{a, b, c\}\}$ & $\{\{b\},\{c\}\}$ & $\{\{a\},\{c\}\}$ \\
\hline F13 & $\{\{a, b\},\{b, c\},\{a, b, c\}\}$ & $\{\{a\},\{b\},\{c\},\{a, c\}\}$ & $\begin{array}{l}\{\{a\},\{b\},\{c\},\{a, b\},\{a, c\}, \\
\{b, c\}\}\end{array}$ & $\begin{array}{l}\{a \mathrm{a}\},\{b\},\{c\},\{a, b\},\{a, c\}, \\
\{b, c\},\{a, b, c\}\}\end{array}$ \\
\hline F15 & $\{\{a, b\},\{a, b, c\}\}$ & $\{\{b\},\{c\},\{a, c\},\{b, c\}\}$ & $\begin{array}{l}\{\{b\},\{c\},\{a, b\},\{a, c\} \\
\{b, c\},\{a, b, c\}\}\end{array}$ & $\{\{a\},\{a, b, c\}\}$ \\
\hline F18 & $\{\{a, b, c\}\}$ & $\{\{c\},\{a, b\},\{a, c\},\{b, c\}\}$ & $\{\{a\},\{b\},\{a, b\},\{b, c\}\}$ & $\begin{array}{l}\{\{a\},\{b\},\{c\},\{a, b\},\{b, c\}, \\
\{a, b, c\}\}\end{array}$ \\
\hline- & $\{\Phi\}$ & $\{\{a, b\},\{a, c\},\{b, c\},\{a, b, c\}\}$ & $\{\{b\},\{c\},\{a, b\},\{a, b, c\}\}$ & $\{\{a\},\{c\},\{b, c\},\{a, b, c\}\}$ \\
\hline
\end{tabular}

Filter chain \# 44 and its STRT spectrum

\begin{tabular}{|l|l|l|l|l|}
\hline F1 & $\begin{array}{l}\{\{a\},\{b\},\{c\},\{a, b\},\{a, c\}, \\
\{b, c\},\{a, b, c\}\}\end{array}$ & $\begin{array}{l}\{\{a\},\{b\},\{c\},\{a, b\},\{a, c\}, \\
\{b, c\},\{a, b, c\}\}\end{array}$ & $\begin{array}{l}\{\{a\},\{b\},\{c\},\{a, b\},\{a, c\}, \\
\{b, c\},\{a, b, c\}\}\end{array}$ & $\begin{array}{l}\{\{a\},\{b\},\{c\},\{a, b\},\{a, c\}, \\
\{b, c\},\{a, b, c\}\}\end{array}$ \\
\hline F4 & $\begin{array}{l}\{\{b\},\{c\},\{a, b\},\{a, c\}, \\
\{b, c\},\{a, b, c\}\}\end{array}$ & $\begin{array}{l}\{\{b\},\{c\},\{a, b\},\{a, c\}, \\
\{b, c\},\{a, b, c\}\}\end{array}$ & $\begin{array}{l}\{\{b\},\{c\},\{a, b\},\{a, c\}, \\
\{b, c\},\{a, b, c\}\}\end{array}$ & $\{\{a\}\}$ \\
\hline F7 & $\begin{array}{l}\{\{c\},\{a, b\},\{a, c\},\{b, c\}, \\
\{a, b, c\}\}\end{array}$ & $\begin{array}{l}\{\{c\},\{a, b\},\{a, c\},\{b, c\}, \\
\{a, b, c\}\}\end{array}$ & $\{\{a\},\{b\}\}$ & $\{\{a\},\{b\},\{c\}\}$ \\
\hline F11 & $\{\{a, b\},\{a, c\},\{b, c\},\{a, b, c\}\}$ & $\{\{a, b\},\{a, c\},\{b, c\},\{a, b, c\}\}$ & $\{\{b\},\{c\}\}$ & $\{\{a\},\{c\}\}$ \\
\hline F13 & $\{\{a, b\},\{b, c\},\{a, b, c\}\}$ & $\{\{a\},\{b\},\{c\},\{a, c\}\}$ & $\begin{array}{l}\{\{a\},\{b\},\{c\},\{a, b\},\{a, c\}, \\
\{b, c\}\}\end{array}$ & $\begin{array}{l}\{a\},\{b\},\{c\},\{a, b\},\{a, c\}, \\
\{b, c\},\{a, b, c\}\}\end{array}$ \\
\hline F17 & $\{\{b, c\},\{a, b, c\}\}$ & $\{\{b\},\{c\},\{a, b\},\{a, c\}\}$ & $\begin{array}{l}\{\{b\},\{c\},\{a, b\},\{a, c\}, \\
\{b, c\},\{a, b, c\}\}\end{array}$ & $\{\{a\},\{a, b, c\}\}$ \\
\hline F18 & $\{\{a, b, c\}\}$ & $\{\{c\},\{a, b\},\{a, c\},\{b, c\}\}$ & $\{a a\},\{b\},\{a, b\},\{b, c\}\}$ & $\begin{array}{l}\{a\},\{b\},\{c\},\{a, b\},\{b, c\}, \\
\{a, b, c\}\}\end{array}$ \\
\hline- & $\{\Phi\}$ & $\{\{a, b\},\{a, c\},\{b, c\},\{a, b, c\}\}$ & $\{\{b\},\{c\},\{b, c\},\{a, b, c\}\}$ & $\{\{a\},\{c\},\{a, b\},\{a, b, c\}\}$ \\
\hline
\end{tabular}

Filter chain \# 45 and its STRT spectrum

\begin{tabular}{|l|l|l|l|l|}
\hline F1 & $\begin{array}{l}\{\{a\},\{b\},\{c\},\{a, b\},\{a, c\}, \\
\{b, c\},\{a, b, c\}\}\end{array}$ & $\begin{array}{l}\{\{a\},\{b\},\{c\},\{a, b\},\{a, c\}, \\
\{b, c\},\{a, b, c\}\}\end{array}$ & $\begin{array}{l}\{\{a\},\{b\},\{c\},\{a, b\},\{a, c\}, \\
\{b, c\},\{a, b, c\}\}\end{array}$ & $\begin{array}{l}\{\{a\},\{b\},\{c\},\{a, b\},\{a, c\}, \\
\{b, c\},\{a, b, c\}\}\end{array}$ \\
\hline F4 & $\begin{array}{l}\{\{b\},\{c\},\{a, b\},\{a, c\}, \\
\{b, c\},\{a, b, c\}\}\end{array}$ & $\begin{array}{l}\{\{b\},\{c\},\{a, b\},\{a, c\}, \\
\{b, c\},\{a, b, c\}\}\end{array}$ & $\begin{array}{l}\{\{b\},\{c\},\{a, b\},\{a, c\}, \\
\{b, c\},\{a, b, c\}\}\end{array}$ & $\{\{a\}\}$ \\
\hline$F 7$ & $\begin{array}{l}\{\{c\},\{a, b\},\{a, c\},\{b, c\}, \\
\{a, b, c\}\}\end{array}$ & $\begin{array}{l}\{\{c\},\{a, b\},\{a, c\},\{b, c\}, \\
\{a, b, c\}\}\end{array}$ & $\{\{a\},\{b\}\}$ & $\{\{a\},\{b\},\{c\}\}$ \\
\hline$F 11$ & $\{\{a, b\},\{a, c\},\{b, c\},\{a, b, c\}\}$ & $\{\{a, b\},\{a, c\},\{b, c\},\{a, b, c\}\}$ & $\{\{b\},\{c\}\}$ & $\{\{a\},\{c\}\}$ \\
\hline$F 14$ & $\{\{a, c\},\{b, c\},\{a, b, c\}\}$ & $\{\{a\},\{b\},\{c\},\{a, b\}\}$ & $\begin{array}{l}\{\{a\},\{b\},\{c\},\{a, b\},\{a, c\}, \\
\{b, c\}\}\end{array}$ & $\begin{array}{l}\{\{a\},\{b\},\{c\},\{a, b\},\{a, c\}, \\
\{b, c\},\{a, b, c\}\}\end{array}$ \\
\hline$F 16$ & $\{\{a, c\},\{a, b, c\}\}$ & $\{\{b\},\{c\},\{a, b\},\{b, c\}\}$ & $\begin{array}{l}\{\{b\},\{c\},\{a, b\},\{a, c\}, \\
\{b, c\},\{a, b, c\}\}\end{array}$ & $\{\{a\},\{a, b, c\}\}$ \\
\hline F18 & $\{\{a, b, c\}\}$ & $\{\{c\},\{a, b\},\{a, c\},\{b, c\}\}$ & $\{\{a\},\{b\},\{a, c\},\{b, c\}\}$ & $\begin{array}{l}\{a b\},\{b\},\{c\},\{a, c\},\{b, c\}, \\
\{a, b, c\}\}\end{array}$ \\
\hline- & $\{\Phi\}$ & $\{\{a, b\},\{a, c\},\{b, c\},\{a, b, c\}\}$ & $\{\{b\},\{c\},\{a, c\},\{a, b, c\}\}$ & $\{\{a\},\{c\},\{b, c c\},\{a, b, c\}\}$ \\
\hline
\end{tabular}


Filter chain \# 46 and its STRT spectrum

\begin{tabular}{|l|l|l|l|l|}
\hline F1 & $\begin{array}{l}\{\{a\},\{b\},\{c\},\{a, b\},\{a, c\}, \\
\{b, c\},\{a, b, c\}\}\end{array}$ & $\begin{array}{l}\{\{a\},\{b\},\{c\},\{a, b\},\{a, c\}, \\
\{b, c\},\{a, b, c\}\}\end{array}$ & $\begin{array}{l}\{\{a\},\{b\},\{c\},\{a, b\},\{a, c\}, \\
\{b, c\},\{a, b, c\}\}\end{array}$ & $\begin{array}{l}\{\{a\},\{b\},\{c\},\{a, b\},\{a, c\}, \\
\{b, c\},\{a, b, c\}\}\end{array}$ \\
\hline F4 & $\begin{array}{l}\{\{b\},\{c\},\{a, b\},\{a, c\},\{b, c\}, \\
\{a, b, c\}\}\end{array}$ & $\begin{array}{l}\{\{b\},\{c\},\{a, b\},\{a, c\}, \\
\{b, c\},\{a, b, c\}\}\end{array}$ & $\begin{array}{l}\{\{b\},\{c\},\{a, b\},\{a, c\}, \\
\{b, c\},\{a, b, c\}\}\end{array}$ & $\{\{a\}\}$ \\
\hline$F 7$ & $\begin{array}{l}\{\{c\},\{a, b\},\{a, c\},\{b, c\}, \\
\{a, b, c\}\}\end{array}$ & $\begin{array}{l}\{\{c\},\{a, b\},\{a, c\},\{b, c\}, \\
\{a, b, c\}\}\end{array}$ & $\{\{a\},\{b\}\}$ & $\{\{a\},\{b\},\{c\}\}$ \\
\hline$F 11$ & $\begin{array}{l}\{\{a, b\},\{a, c\},\{b, c\}, \\
\{a, b, c\}\}\end{array}$ & $\begin{array}{l}\{\{a, b\},\{a, c\},\{b, c\}, \\
\{a, b, c\}\}\end{array}$ & $\{\{b\},\{c\}\}$ & $\{\{a\},\{c\}\}$ \\
\hline$F 14$ & $\{\{a, c\},\{b, c\},\{a, b, c\}\}$ & $\{\{a\},\{b\},\{c\},\{a, b\}\}$ & $\begin{array}{l}\{\{a\},\{b\},\{c\},\{a, b\},\{a, c\}, \\
\{b, c\}\}\end{array}$ & $\begin{array}{l}\{\{a\},\{b\},\{c\},\{a, b\},\{a, c\}, \\
\{b, c\},\{a, b, c\}\}\end{array}$ \\
\hline$F 17$ & $\{\{b, c\},\{a, b, c\}\}$ & $\{\{b\},\{c\},\{a, b\},\{a, c\}\}$ & $\begin{array}{l}\{\{b\},\{c\},\{a, b\},\{a, c\}, \\
\{b, c\},\{a, b, c\}\}\end{array}$ & $\{\{a\},\{a, b, c\}\}$ \\
\hline F18 & $\{\{a, b, c\}\}$ & $\{\{c\},\{a, b\},\{a, c\},\{b, c\}\}$ & $\{\{a\},\{b\},\{a, c\},\{b, c\}\}$ & $\begin{array}{l}\{\{a\},\{b\},\{c\},\{a, c\},\{b, c\}, \\
\{a, b, c\}\}\end{array}$ \\
\hline- & $\{\Phi\}$ & $\{\{a, b\},\{a, c\},\{b, c\},\{a, b, c\}\}$ & $\{\{b\},\{c\},\{b, c\},\{a, b, c\}\}$ & $\{\{a\},\{c\},\{a, c\},\{a, b, c\}\}$ \\
\hline
\end{tabular}

Filter chain \# 47 and its STRT spectrum

\begin{tabular}{|c|c|c|c|c|}
\hline F1 & $\begin{array}{l}\{\{a\},\{b\},\{c\},\{a, b\},\{a, c\}, \\
\{b, c\},\{a, b, c\}\}\end{array}$ & $\begin{array}{l}\{\{a\},\{b\},\{c\},\{a, b\},\{a, c\} \\
\{b, c\},\{a, b, c\}\}\end{array}$ & $\begin{array}{l}\{\{a\},\{b\},\{c\},\{a, b\},\{a, c\} \\
\{b, c\},\{a, b, c\}\}\end{array}$ & $\begin{array}{l}\{\{a\},\{b\},\{c\},\{a, b\},\{a, c\}, \\
\{b, c\},\{a, b, c\}\}\end{array}$ \\
\hline F4 & $\begin{array}{l}\{\{b\},\{c\},\{a, b\},\{a, c\} \\
\{b, c\},\{a, b, c\}\}\end{array}$ & $\begin{array}{l}\{\{b\},\{c\},\{a, b\},\{a, c\},\{b, c\}, \\
\{a, b, c\}\}\end{array}$ & $\begin{array}{l}\{\{b\},\{c\},\{a, b\},\{a, c\}, \\
\{b, c\},\{a, b, c\}\}\end{array}$ & $\{\{a\}\}$ \\
\hline F7 & $\begin{array}{l}\{\{c\},\{a, b\},\{a, c\},\{b, c\}, \\
\{a, b, c\}\}\end{array}$ & $\begin{array}{l}\{\{c\},\{a, b\},\{a, c\},\{b, c\} \\
\{a, b, c\}\}\end{array}$ & $\{\{a\},\{b\}\}$ & $\{\{a\},\{b\},\{a, b\}\}$ \\
\hline $\mathrm{F} 10$ & $\{\{c\},\{a, c\},\{b, c\},\{a, b, c\}\}$ & $\{\{c\},\{a, c\},\{b, c\},\{a, b, c\}\}$ & $\{\{b\},\{a, b\}\}$ & $\{\{a\},\{a, b\}\}$ \\
\hline $\mathrm{F} 14$ & $\{\{a, c\},\{b, c\},\{a, b, c\}\}$ & $\{\{a\},\{b\},\{c\},\{a, b\}\}$ & $\begin{array}{l}\{\{a\},\{b\},\{c\},\{a, b\},\{a, c\}, \\
\{b, c\}\}\end{array}$ & $\begin{array}{l}\{a a\},\{b\},\{c\},\{a, b\},\{a, c\}, \\
\{b, c\},\{a, b, c\}\}\end{array}$ \\
\hline F16 & $\{\{a, c\},\{a, b, c\}\}$ & $\{\{b\},\{c\},\{a, b\},\{b, c\}\}$ & $\begin{array}{l}\{\{b\},\{c\},\{a, b\},\{a, c\} \\
\{b, c\},\{a, b, c\}\}\end{array}$ & $\{\{a\},\{a, b, c\}\}$ \\
\hline F18 & $\{\{a, b, c\}\}$ & $\{\{c\},\{a, b\},\{a, c\},\{b, c\}\}$ & $\{\{a\},\{b\},\{a, c\},\{b, c\}\}$ & $\begin{array}{l}\{\{a\},\{b\},\{a, b\},\{a, c\} \\
\{b, c\},\{a, b, c\}\}\end{array}$ \\
\hline- & $\{\Phi\}$ & $\{\{c\},\{a, c\},\{b, c\},\{a, b, c\}\}$ & $\{\{b\},\{a, b\},\{a, c\},\{a, b, c\}\}$ & $\{\{a\},\{a, b\},\{b, c\},\{a, b, c\}\}$ \\
\hline
\end{tabular}

Filter chain \# 48 and its STRT spectrum

\begin{tabular}{|l|l|l|l|l|}
\hline F1 & $\begin{array}{l}\{\{a\},\{b\},\{c\},\{a, b\},\{a, c\}, \\
\{b, c\},\{a, b, c\}\}\end{array}$ & $\begin{array}{l}\{\{a\},\{b\},\{c\},\{a, b\},\{a, c\}, \\
\{b, c\},\{a, b, c\}\}\end{array}$ & $\begin{array}{l}\{\{a\},\{b\},\{c\},\{a, b\},\{a, c\}, \\
\{b, c\},\{a, b, c\}\}\end{array}$ & $\begin{array}{l}\{\{a\},\{b\},\{c\},\{a, b\},\{a, c\}, \\
\{b, c\},\{a, b, c\}\}\end{array}$ \\
\hline F4 & $\begin{array}{l}\{\{b\},\{c\},\{a, b\},\{a, c\}, \\
\{b, c\},\{a, b, c\}\}\end{array}$ & $\begin{array}{l}\{\{b\},\{c\},\{a, b\},\{a, c\}, \\
\{b, c\},\{a, b, c\}\}\end{array}$ & $\begin{array}{l}\{\{b\},\{c\},\{a, b\},\{a, c\}, \\
\{b, c\},\{a, b, c\}\}\end{array}$ & $\{\{a\}\}$ \\
\hline F7 & $\begin{array}{l}\{\{c\},\{a, b\},\{a, c\},\{b, c\}, \\
\{a, b, c\}\}\end{array}$ & $\begin{array}{l}\{\{c\},\{a, b\},\{a, c\},\{b, c\}, \\
\{a, b, c\}\}\end{array}$ & $\{\{a\},\{b\}\}$ & $\{a b\},\{b\},\{a, b\}\}$ \\
\hline$F 10$ & $\{\{c\},\{a, c\},\{b, c\},\{a, b, c\}\}$ & $\{\{c\},\{a, c\},\{b, c\},\{a, b, c\}\}$ & $\{\{b\},\{a, b\}\}$ & $\{\{a\},\{a, b\}\}$ \\
\hline$F 14$ & $\{\{a, c\},\{b, c\},\{a, b, c\}\}$ & $\{\{a\},\{b\},\{c\},\{a, b\}\}$ & $\begin{array}{l}\{a a\},\{b\},\{c\},\{a, b\},\{a, c\}, \\
\{b, c\}\}\end{array}$ & $\begin{array}{l}\{\{a\},\{b\},\{c\},\{a, b\},\{a, c\}, \\
\{b, c\},\{a, b, c\}\}\end{array}$ \\
\hline F17 & $\{\{b, c\},\{a, b, c\}\}$ & $\{\{b\},\{c\},\{a, b\},\{a, c\}\}$ & $\begin{array}{l}\{\{b\},\{c\},\{a, b\},\{a, c\}, \\
\{b, c\},\{a, b, c\}\}\end{array}$ & $\{\{a\},\{a, b, c\}\}$ \\
\hline F18 & $\{\{a, b, c\}\}$ & $\{\{c\},\{a, b\},\{a, c\},\{b, c\}\}$ & $\{\{a\},\{b\},\{a, c\},\{b, c\}\}$ & $\begin{array}{l}\{\{a\},\{b\},\{a, b\},\{a, c\},\{b, c\}, \\
\{a, b, c\}\}\end{array}$ \\
\hline- & $\{\Phi\}$ & $\{\{c\},\{a, c\},\{b, c\},\{a, b, c\}\}$ & $\{\{b\},\{a, b\},\{b, c\},\{a, b, c\}\}$ & $\{\{a\},\{a, b\},\{a, c\},\{a, b, c\}\}$ \\
\hline
\end{tabular}

Consider a finite set $X=\{a, b, c, d\}$. Then its power set is $\{\{\},\{a\},\{b\},\{c\},\{d\},\{a, b\},\{a, c\},\{a, d\},\{b, c\},\{b, d\}$, $\{c, d\},\{a, b, c\},\{a, b, d\},\{a, c, d\},\{b, c, d\},\{a, b, c, d\}\}$. One can construct a filter set $F$ whose elements satisfy the following property: 'Any element of $F$ ensures the presence of all its super sets present in the power set of $X$. One can construct 166 topological filters from the ground set $X=\{a, b, c, d\}$. This list is given in table 2 . 
Table 2: List of topological filters defined over $X=\{a, b, c, d\}$

\begin{tabular}{|c|c|c|}
\hline $\begin{array}{l}\text { Filter } \\
\text { No. }\end{array}$ & Filter Elements & Cardinality \\
\hline F1 & $\{\{a\},\{b\},\{c\},\{d\},\{a, b\},\{a, c\},\{a, d\},\{b, c\},\{b, d\},\{c, d\},\{a, b, c\},\{a, b, d\},\{a, c, d\},\{b, c, d\},\{a, b, c, d\}\}$ & 15 \\
\hline F2 & $\{\{a\},\{b\},\{c\},\{a, b\},\{a, c\},\{a, d\},\{b, c\},\{b, d\},\{c, d\},\{a, b, c\},\{a, b, d\},\{a, c, d\},\{b, c, d\},\{a, b, c, d\}\}$ & 14 \\
\hline F3 & $\{\{a\},\{b\},\{d\},\{a, b\},\{a, c\},\{a, d\},\{b, c\},\{b, d\},\{c, d\},\{a, b, c\},\{a, b, d\},\{a, c, d\},\{b, c, d\},\{a, b, c, d\}\}$ & 14 \\
\hline F4 & $\{\{a\},\{c\},\{d\},\{a, b\},\{a, c\},\{a, d\},\{b, c\},\{b, d\},\{c, d\},\{a, b, c\},\{a, b, d\},\{a, c, d\},\{b, c, d\},\{a, b, c, d\}\}$ & 14 \\
\hline F5 & $\{\{b\},\{c\},\{d\},\{a, b\},\{a, c\},\{a, d\},\{b, c\},\{b, d\},\{c, d\},\{a, b, c\},\{a, b, d\},\{a, c, d\},\{b, c, d\},\{a, b, c, d\}\}$ & 14 \\
\hline F6 & $\{\{a\},\{b\},\{a, b\},\{a, c\},\{a, d\},\{b, c\},\{b, d\},\{c, d\},\{a, b, c\},\{a, b, d\},\{a, c, d\},\{b, c, d\},\{a, b, c, d\}\}$ & 13 \\
\hline F7 & $\{\{a\},\{c\},\{a, b\},\{a, c\},\{a, d\},\{b, c\},\{b, d\},\{c, d\},\{a, b, c\},\{a, b, d\},\{a, c, d\},\{b, c, d\},\{a, b, c, d\}\}$ & 13 \\
\hline F8 & $\{\{b\},\{c\},\{a, b\},\{a, c\},\{a, d\},\{b, c\},\{b, d\},\{c, d\},\{a, b, c\},\{a, b, d\},\{a, c, d\},\{b, c, d\},\{a, b, c, d\}\}$ & 13 \\
\hline F9 & $\{\{a\},\{d\},\{a, b\},\{a, c\},\{a, d\},\{b, c\},\{b, d\},\{c, d\},\{a, b, c\},\{a, b, d\},\{a, c, d\},\{b, c, d\},\{a, b, c, d\}\}$ & 13 \\
\hline F10 & $\{\{b\},\{d\},\{a, b\},\{a, c\},\{a, d\},\{b, c\},\{b, d\},\{c, d\},\{a, b, c\},\{a, b, d\},\{a, c, d\},\{b, c, d\},\{a, b, c, d\}\}$ & 13 \\
\hline F11 & $\{\{c\},\{d\},\{a, b\},\{a, c\},\{a, d\},\{b, c\},\{b, d\},\{c, d\},\{a, b, c\},\{a, b, d\},\{a, c, d\},\{b, c, d\},\{a, b, c, d\}\}$ & 13 \\
\hline F12 & $\{\{a\},\{b\},\{a, b\},\{a, c\},\{a, d\},\{b, c\},\{b, d\},\{a, b, c\},\{a, b, d\},\{a, c, d\},\{b, c, d\},\{a, b, c, d\}\}$ & 12 \\
\hline F13 & $\{\{a\},\{a, b\},\{a, c\},\{a, d\},\{b, c\},\{b, d\}\{c, d\},\{a, b, c\},\{a, b, d\},\{a, c, d\},\{b, c, d\},\{a, b, c, d\}\}$ & 12 \\
\hline F14 & $\{\{a\},\{c\},\{a, b\},\{a, c\},\{a, d\},\{b, c\},\{c, d\},\{a, b, c\},\{a, b, d\},\{a, c, d\},\{b, c, d\},\{a, b, c, d\}\}$ & 12 \\
\hline F15 & $\{\{b\},\{a, b\},\{a, c\},\{a, d\},\{b, c\},\{b, d\},\{c, d\},\{a, b, c\},\{a, b, d\},\{a, c, d\},\{b, c, d\},\{a, b, c, d\}\}$ & 12 \\
\hline F16 & $\{\{b\},\{c\},\{a, b\},\{a, c\},\{b, c\},\{b, d\},\{c, d\},\{a, b, c\},\{a, b, d\},\{a, c, d\},\{b, c, d\},\{a, b, c, d\}\}$ & 12 \\
\hline F17 & $\{\{c\},\{a, b\},\{a, c\},\{a, d\},\{b, c\},\{b, d\},\{c, d\},\{a, b, c\},\{a, b, d\},\{a, c, d\},\{b, c, d\},\{a, b, c, d\}\}$ & 12 \\
\hline F18 & $\{\{\mathrm{a}\},\{\mathrm{d}\},\{\mathrm{a}, \mathrm{b}\},\{\mathrm{a}, \mathrm{c}\},\{\mathrm{a}, \mathrm{d}\},\{\mathrm{b}, \mathrm{d}\},\{\mathrm{c}, \mathrm{d}\},\{\mathrm{a}, \mathrm{b}, \mathrm{c}\},\{\mathrm{a}, \mathrm{b}, \mathrm{d}\},\{\mathrm{a}, \mathrm{c}, \mathrm{d}\},\{\mathrm{b}, \mathrm{c}, \mathrm{d}\},\{\mathrm{a}, \mathrm{b}, \mathrm{c}, \mathrm{d}\}\}$ & 12 \\
\hline F19 & $\{\{d\},\{a, b\},\{a, c\},\{a, d\},\{b, c\},\{b, d\},\{c, d\},\{a, b, c\},\{a, b, d\},\{a, c, d\},\{b, c, d\},\{a, b, c, d\}\}$ & 12 \\
\hline F20 & $\{\{b\},\{d\},\{a, b\},\{a, d\},\{b, c\},\{b, d\},\{c, d\},\{a, b, c\},\{a, b, d\},\{a, c, d\},\{b, c, d\},\{a, b, c, d\}\}$ & 12 \\
\hline F21 & $\{\{c\},\{d\},\{a, c\},\{a, d\},\{b, c\},\{b, d\},\{c, d\},\{a, b, c\},\{a, b, d\},\{a, c, d\},\{b, c, d\},\{a, b, c, d\}\}$ & 12 \\
\hline F22 & $\{\{a\},\{a, b\},\{a, c\},\{a, d\},\{b, c\},\{b, d\},\{a, b, c\},\{a, b, d\},\{a, c, d\},\{b, c, d\},\{a, b, c, d\}\}$ & 11 \\
\hline F23 & $\{\{a\},\{a, b\},\{a, c\},\{a, d\},\{b, d\},\{c, d\},\{a, b, c\},\{a, b, d\},\{a, c, d\},\{b, c, d\},\{a, b, c, d\}\}$ & 11 \\
\hline F24 & $\{\{a\},\{a, b\},\{a, c\},\{a, d\},\{b, c\},\{c, d\},\{a, b, c\},\{a, b, d\},\{a, c, d\},\{b, c, d\},\{a, b, c, d\}\}$ & 11 \\
\hline F25 & $\{\{b\},\{a, b\},\{a, d\},\{b, c\},\{b, d\},\{c, d\},\{a, b, c\},\{a, b, d\},\{a, c, d\},\{b, c, d\},\{a, b, c, d\}\}$ & 11 \\
\hline F26 & $\{\{b\},\{a, b\},\{a, c\},\{b, c\},\{b, d\},\{c, d\},\{a, b, c\},\{a, b, d\},\{a, c, d\},\{b, c, d\},\{a, b, c, d\}\}$ & 11 \\
\hline F27 & $\{\{a, b\},\{a, c\},\{a, d\},\{b, c\},\{b, d\},\{c, d\},\{a, b, c\},\{a, b, d\},\{a, c, d\},\{b, c, d\},\{a, b, c, d\}\}$ & 11 \\
\hline F28 & $\{\{b\},\{a, b\},\{a, c\},\{a, d\},\{b, c\},\{b, d\},\{a, b, c\},\{a, b, d\},\{a, c, d\},\{b, c, d\},\{a, b, c, d\}\}$ & 11 \\
\hline F29 & $\{\{c\},\{a, c\},\{a, d\},\{b, c\},\{b, d\},\{c, d\},\{a, b, c\},\{a, b, d\},\{a, c, d\},\{b, c, d\},\{a, b, c, d\}\}$ & 11 \\
\hline F30 & $\{\{c\},\{a, b\},\{a, c\},\{b, c\},\{b, d\},\{c, d\},\{a, b, c\},\{a, b, d\},\{a, c, d\},\{b, c, d\},\{a, b, c, d\}\}$ & 11 \\
\hline F31 & $\{\{c\},\{a, b\},\{a, c\},\{a, d\},\{b, c\},\{c, d\},\{a, b, c\},\{a, b, d\},\{a, c, d\},\{b, c, d\},\{a, b, c, d\}\}$ & 11 \\
\hline F32 & $\{\{d\},\{a, c\},\{a, d\},\{b, c\},\{b, d\},\{c, d\},\{a, b, c\},\{a, b, d\},\{a, c, d\},\{b, c, d\},\{a, b, c, d\}\}$ & 11 \\
\hline F33 & $\{\{d\},\{a, b\},\{a, d\},\{b, c\},\{b, d\},\{c, d\},\{a, b, c\},\{a, b, d\},\{a, c, d\},\{b, c, d\},\{a, b, c, d\}\}$ & 11 \\
\hline F34 & $\{\{d\},\{a, b\},\{a, c\},\{a, d\},\{b, d\},\{c, d\},\{a, b, c\},\{a, b, d\},\{a, c, d\},\{b, c, d\},\{a, b, c, d\}\}$ & 11 \\
\hline F35 & $\{\{a\},\{a, b\},\{a, c\},\{a, d\},\{b, d\},\{a, b, c\},\{a, b, d\},\{a, c, d\},\{b, c, d\},\{a, b, c, d\}\}$ & 10 \\
\hline F36 & $\{\{a\},\{a, b\},\{a, c\},\{a, d\},\{b, c\},\{a, b, c\},\{a, b, d\},\{a, c, d\},\{b, c, d\},\{a, b, c, d\}\}$ & 10 \\
\hline F37 & $\{\{a\},\{a, b\},\{a, c\},\{a, d\},\{c, d\},\{a, b, c\},\{a, b, d\},\{a, c, d\},\{b, c, d\},\{a, b, c, d\}\}$ & 10 \\
\hline F38 & $\{\{b\},\{a, b\},\{b, c\},\{b, d\},\{c, d\},\{a, b, c\},\{a, b, d\},\{a, c, d\},\{b, c, d\},\{a, b, c, d\}\}$ & 10 \\
\hline F39 & $\{\{b\},\{a, b\},\{a, d\},\{b, c\},\{b, d\},\{a, b, c\},\{a, b, d\},\{a, c, d\},\{b, c, d\},\{a, b, c, d\}\}$ & 10 \\
\hline F40 & $\{\{b\},\{a, b\},\{a, c\},\{b, c\},\{b, d\},\{a, b, c\},\{a, b, d\},\{a, c, d\},\{b, c, d\},\{a, b, c, d\}\}$ & 10 \\
\hline F41 & $\{\{a, c\},\{a, d\},\{b, c\},\{b, d\},\{c, d\},\{a, b, c\},\{a, b, d\},\{a, c, d\},\{b, c, d\},\{a, b, c, d\}\}$ & 10 \\
\hline F42 & $\{\{a, b\},\{a, d\},\{b, c\},\{b, d\},\{c, d\},\{a, b, c\},\{a, b, d\},\{a, c, d\},\{b, c, d\},\{a, b, c, d\}\}$ & 10 \\
\hline F43 & $\{\{a, b\},\{a, c\},\{b, c\}, b, d\},\{c, d\},\{a, b, c\},\{a, b, d\},\{a, c, d\},\{b, c, d\},\{a, b, c, d\}\}$ & 10 \\
\hline F44 & $\{\{a, b\},\{a, c\},\{a, d\},\{b, d\},\{c, d\},\{a, b, c\},\{a, b, d\},\{a, c, d\},\{b, c, d\},\{a, b, c, d\}\}$ & 10 \\
\hline F45 & $\{\{a, b\},\{a, c\},\{a, d\},\{b, c\},\{c, d\},\{a, b, c\},\{a, b, d\},\{a, c, d\},\{b, c, d\},\{a, b, c, d\}\}$ & 10 \\
\hline F46 & $\{\{a, b\},\{a, c\},\{a, d\},\{b, c\},\{b, d\},\{a, b, c\},\{a, b, d\},\{a, c, d\},\{b, c, d\},\{a, b, c, d\}\}$ & 10 \\
\hline F47 & $\{\{c\},,\{a, c\},\{b, c\},\{b, d\},\{c, d\},\{a, b, c\},\{a, b, d\},\{a, c, d\},\{b, c, d\},\{a, b, c, d\}\}$ & 10 \\
\hline F48 & $\{\{c\},\{a, c\},\{a, d\},\{b, c\},\{c, d\},\{a, b, c\},\{a, b, d\},\{a, c, d\},\{b, c, d\},\{a, b, c, d\}\}$ & 10 \\
\hline F49 & $\{\{c\},\{a, b\},\{a, c\},\{b, c\},\{c, d\},\{a, b, c\},\{a, b, d\},\{a, c, d\},\{b, c, d\},\{a, b, c, d\}\}$ & 10 \\
\hline F50 & $\{\{d\},\{a, c\},\{a, d\},\{b, d\},\{c, d\},\{a, b, c\},\{a, b, d\},\{a, c, d\},\{b, c, d\},\{a, b, c, d\}\}$ & 10 \\
\hline F51 & $\{\{d\},\{a, d\},\{b, c\},\{b, d\},\{c, d\},\{a, b, c\},\{a, b, d\},\{a, c, d\},\{b, c, d\},\{a, b, c, d\}\}$ & 10 \\
\hline F52 & $\{\{\mathrm{d}\},\{\mathrm{a}, \mathrm{b}\},\{\mathrm{a}, \mathrm{d}\},\{\mathrm{b}, \mathrm{d}\},\{\mathrm{c}, \mathrm{d}\},\{\mathrm{a}, \mathrm{b}, \mathrm{c}\},\{\mathrm{a}, \mathrm{b}, \mathrm{d}\},\{\mathrm{a}, \mathrm{c}, \mathrm{d}\},\{\mathrm{b}, \mathrm{c}, \mathrm{d}\},\{\mathrm{a}, \mathrm{b}, \mathrm{c}, \mathrm{d}\}\}$ & 10 \\
\hline F53 & $\{\{a\},\{a, b\},\{a, c\},\{a, d\},\{a, b, c\},\{a, b, d\},\{a, c, d\},\{b, c, d\},\{a, b, c, d\}\}$ & 9 \\
\hline F54 & $\{\{b\},\{a, b\},\{b, c\},\{b, d\},\{a, b, c\},\{a, b, d\},\{a, c, d\},\{b, c, d\},\{a, b, c, d\}\}$ & 9 \\
\hline F55 & $\{\{a, d\},\{b, c\},\{b, d\},\{c, d\},\{a, b, c\},\{a, b, d\},\{a, c, d\},\{b, c, d\},\{a, b, c, d\}\}$ & 9 \\
\hline F56 & $\{\{a, d\},\{b, c\},\{b, d\},\{c, d\},\{a, b, c\},\{a, b, d\},\{a, c, d\},\{b, c, d\},\{a, b, c, d\}\}$ & 9 \\
\hline
\end{tabular}




\begin{tabular}{|c|c|c|}
\hline F57 & $\{\{a, c\},\{a, d\},\{b, d\},\{c, d\},\{a, b, c\},\{a, b, d\},\{a, c, d\},\{b, c, d\},\{a, b, c, d\}\}$ & 9 \\
\hline F58 & $\{\{a, c\},\{a, d\},\{b, c\},\{c, d\},\{a, b, c\},\{a, b, d\},\{a, c, d\},\{b, c, d\},\{a, b, c, d\}\}$ & 9 \\
\hline F59 & $\{\{a, c\},\{a, d\},\{b, c\},\{b, d\},\{a, b, c\},\{a, b, d\},\{a, c, d\},\{b, c, d\},\{a, b, c, d\}\}$ & 9 \\
\hline F60 & $\{\{a, b\},\{b, c\},\{b, d\},\{c, d\},\{a, b, c\},\{a, b, d\},\{a, c, d\},\{b, c, d\},\{a, b, c, d\}\}$ & 9 \\
\hline F61 & $\{\{a, b\},\{a, d\},\{b, d\},\{c, d\},\{a, b, c\},\{a, b, d\},\{a, c, d\},\{b, c, d\},\{a, b, c, d\}\}$ & 9 \\
\hline F62 & $\{\{a, b\},\{a, d\},\{b, c\},\{c, d\},\{a, b, c\},\{a, b, d\},\{a, c, d\},\{b, c, d\},\{a, b, c, d\}\}$ & 9 \\
\hline F63 & $\{\{a, b\},\{a, d\},\{b, c\},\{b, d\},\{a, b, c\},\{a, b, d\},\{a, c, d\},\{b, c, d\},\{a, b, c, d\}\}$ & 9 \\
\hline F64 & $\{\{a, b\},\{a, c\},\{b, d\},\{c, d\},\{a, b, c\},\{a, b, d\},\{a, c, d\},\{b, c, d\},\{a, b, c, d\}\}$ & 9 \\
\hline F65 & $\{\{a, b\},\{a, c\},\{b, c\},\{c, d\},\{a, b, c\},\{a, b, d\},\{a, c, d\},\{b, c, d\},\{a, b, c, d\}\}$ & 9 \\
\hline F66 & $\{\{a, b\},\{a, c\},\{b, c\},\{b, d\},\{a, b, c\},\{a, b, d\},\{a, c, d\},\{b, c, d\},\{a, b, c, d\}\}$ & 9 \\
\hline F67 & $\{\{a, b\},\{a, c\},\{a, d\},\{c, d\},\{a, b, c\},\{a, b, d\},\{a, c, d\},\{b, c, d\},\{a, b, c, d\}\}$ & 9 \\
\hline F68 & $\{\{a, b\},\{a, c\},\{a, d\},\{b, c\},\{a, b, c\},\{a, b, d\},\{a, c, d\},\{b, c, d\},\{a, b, c, d\}\}$ & 9 \\
\hline F69 & $\{\{a, b\},\{a, c\},\{a, d\},\{b, d\},\{a, b, c\},\{a, b, d\},\{a, c, d\},\{b, c, d\},\{a, b, c, d\}\}$ & 9 \\
\hline F70 & $\{\{c\},\{a, c\},\{b, c\},\{c, d\},\{a, b, c\},\{a, b, d\},\{a, c, d\},\{b, c, d\},\{a, b, c, d\}\}$ & 9 \\
\hline F71 & $\{\{d\},\{a, d\},\{b, d\},\{c, d\},\{a, b, c\},\{a, b, d\},\{a, c, d\},\{b, c, d\},\{a, b, c, d\}\}$ & 9 \\
\hline F72 & $\{\{a\},\{a, b\},\{a, c\},\{a, d\},\{a, b, c\},\{a, b, d\},\{a, c, d\},\{a, b, c, d\}\}$ & 8 \\
\hline F73 & $\{\{b\},\{a, b\},\{b, c\},\{b, d\},\{a, b, c\},\{a, b, d\},\{b, c, d\},\{a, b, c, d\}\}$ & 8 \\
\hline F74 & $\{\{b, c\},\{b, d\},\{c, d\},\{a, b, c\},\{a, b, d\},\{a, c, d\},\{b, c, d\},\{a, b, c, d\}\}$ & 8 \\
\hline F75 & $\{\{a, d\},\{b, d\},\{c, d\},\{a, b, c\},\{a, b, d\},\{a, c, d\},\{b, c, d\},\{a, b, c, d\}\}$ & 8 \\
\hline F76 & $\{\{a, d\},\{b, c\},\{c, d\},\{a, b, c\},\{a, b, d\},\{a, c, d\},\{b, c, d\},\{a, b, c, d\}\}$ & 8 \\
\hline F77 & $\{\{a, d\},\{b, c\},\{b, d\},\{a, b, c\},\{a, b, d\},\{a, c, d\},\{b, c, d\},\{a, b, c, d\}\}$ & 8 \\
\hline F78 & $\{\{a, c\},\{b, c\},\{b, d\},\{a, b, c\},\{a, b, d\},\{a, c, d\},\{b, c, d\},\{a, b, c, d\}\}$ & 8 \\
\hline F79 & $\{\{a, c\},\{b, c\},\{c, d\},\{a, b, c\},\{a, b, d\},\{a, c, d\},\{b, c, d\},\{a, b, c, d\}\}$ & 8 \\
\hline F80 & $\{\{a, c\},\{a, d\},\{c, d\},\{a, b, c\},\{a, b, d\},\{a, c, d\},\{b, c, d\},\{a, b, c, d\}\}$ & 8 \\
\hline F81 & $\{\{a, c\},\{a, d\},\{b, d\},\{a, b, c\},\{a, b, d\},\{a, c, d\},\{b, c, d\},\{a, b, c, d\}\}$ & 8 \\
\hline F82 & $\{\{a, c\},\{a, d\},\{b, c\},\{a, b, c\},\{a, b, d\},\{a, c, d\},\{b, c, d\},\{a, b, c, d\}\}$ & 8 \\
\hline F83 & $\{\{a, b\},\{b, d\},\{c, d\},\{a, b, c\},\{a, b, d\},\{a, c, d\},\{b, c, d\},\{a, b, c, d\}\}$ & 8 \\
\hline F84 & $\{\{a, b\},\{b, c\},\{c, d\},\{a, b, c\},\{a, b, d\},\{a, c, d\},\{b, c, d\},\{a, b, c, d\}\}$ & 8 \\
\hline F85 & $\{\{a, b\},\{b, c\},\{b, d\},\{a, b, c\},\{a, b, d\},\{a, c, d\},\{b, c, d\},\{a, b, c, d\}\}$ & 8 \\
\hline F86 & $\{\{a, b\},\{a, d\},\{c, d\},\{a, b, c\},\{a, b, d\},\{a, c, d\},\{b, c, d\},\{a, b, c, d\}\}$ & 8 \\
\hline F87 & $\{\{a, b\},\{a, d\},\{b, d\},\{a, b, c\},\{a, b, d\},\{a, c, d\},\{b, c, d\},\{a, b, c, d\}\}$ & 8 \\
\hline F88 & $\{\{a, b\},\{a, d\},\{b, c\},\{a, b, c\},\{a, b, d\},\{a, c, d\},\{b, c, d\},\{a, b, c, d\}\}$ & 8 \\
\hline F89 & $\{\{a, c\},\{b, d\},\{c, d\},\{a, b, c\},\{a, b, d\},\{a, c, d\},\{b, c, d\},\{a, b, c, d\}\}$ & 8 \\
\hline F90 & $\{\{a, b\},\{a, c\},\{c, d\},\{a, b, c\},\{a, b, d\},\{a, c, d\},\{b, c, d\},\{a, b, c, d\}\}$ & 8 \\
\hline F91 & $\{\{a, b\},\{a, c\},\{b, d\},\{a, b, c\},\{a, b, d\},\{a, c, d\},\{b, c, d\},\{a, b, c, d\}\}$ & 8 \\
\hline F92 & $\{\{a, b\},\{a, c\},\{b, c\},\{a, b, c\},\{a, b, d\},\{a, c, d\},\{b, c, d\},\{a, b, c, d\}\}$ & 8 \\
\hline F93 & $\{\{a, b\},\{a, c\},\{a, d\},\{a, b, c\},\{a, b, d\},\{a, c, d\},\{b, c, d\},\{a, b, c, d\}\}$ & 8 \\
\hline F94 & $\{\{c\},\{a, c\},\{b, c\},\{c, d\},\{a, b, c\},\{a, c, d\},\{b, c, d\},\{a, b, c, d\}\}$ & 8 \\
\hline F95 & $\{\{d\},\{a, d\},\{b, d\},\{c, d\},\{a, b, d\},\{a, c, d\},\{b, c, d\},\{a, b, c, d\}\}$ & 8 \\
\hline F96 & $\{\{a, d\},\{b, d\},\{c, d\},\{a, b, d\},\{a, c, d\},\{b, c, d\},\{a, b, c, d\}\}$ & 7 \\
\hline F97 & $\{\{a, c\},\{b, c\},\{c, d\},\{a, b, c\},\{a, c, d\},\{b, c, d\},\{a, b, c, d\}\}$ & 7 \\
\hline F98 & $\{\{b, d\},\{c, d\},\{a, b, c\},\{a, b, d\},\{a, c, d\},\{b, c, d\},\{a, b, c, d\}\}$ & 7 \\
\hline F99 & $\{\{b, c\},\{c, d\},\{a, b, c\},\{a, b, d\},\{a, c, d\},\{b, c, d\},\{a, b, c, d\}\}$ & 7 \\
\hline F100 & $\{\{b, c\},\{b, d\},\{a, b, c\},\{a, b, d\},\{a, c, d\},\{b, c, d\},\{a, b, c, d\}\}$ & 7 \\
\hline F101 & $\{\{a, d\},\{c, d\},\{a, b, c\},\{a, b, d\},\{a, c, d\},\{b, c, d\},\{a, b, c, d\}\}$ & 7 \\
\hline F102 & $\{\{a, d\},\{b, d\},\{a, b, c\},\{a, b, d\},\{a, c, d\},\{b, c, d\},\{a, b, c, d\}\}$ & 7 \\
\hline F103 & $\{\{a, d\},\{b, c\},\{a, b, c\},\{a, b, d\},\{a, c, d\},\{b, c, d\},\{a, b, c, d\}\}$ & 7 \\
\hline F104 & $\{\{a, c\},\{c, d\},\{a, b, c\},\{a, b, d\},\{a, c, d\},\{b, c, d\},\{a, b, c, d\}\}$ & 7 \\
\hline F105 & $\{\{a, c\},\{b, d\},\{a, b, c\},\{a, b, d\},\{a, c, d\},\{b, c, d\},\{a, b, c, d\}\}$ & 7 \\
\hline F106 & $\{\{a, c\},\{b, c\},\{a, b, c\},\{a, b, d\},\{a, c, d\},\{b, c, d\},\{a, b, c, d\}\}$ & 7 \\
\hline F107 & $\{\{a, c\},\{a, d\},\{a, b, c\},\{a, b, d\},\{a, c, d\},\{b, c, d\},\{a, b, c, d\}\}$ & 7 \\
\hline F108 & $\{\{a, b\},\{c, d\},\{a, b, c\},\{a, b, d\},\{a, c, d\},\{b, c, d\},\{a, b, c, d\}\}$ & 7 \\
\hline F109 & $\{\{a, b\},\{b, d\},\{a, b, c\},\{a, b, d\},\{a, c, d\},\{b, c, d\},\{a, b, c, d\}\}$ & 7 \\
\hline $\mathrm{F} 110$ & $\{\{a, b\},\{b, c\},\{a, b, c\},\{a, b, d\},\{a, c, d\},\{b, c, d\},\{a, b, c, d\}\}$ & 7 \\
\hline F111 & $\{\{a, b\},\{a, d\},\{a, b, c\},\{a, b, d\},\{a, c, d\},\{b, c, d\},\{a, b, c, d\}\}$ & 7 \\
\hline F112 & $\{\{a, b\},\{a, c\},\{a, b, c\},\{a, b, d\},\{a, c, d\},\{b, c, d\},\{a, b, c, d\}\}$ & 7 \\
\hline $\mathrm{F} 113$ & $\{\{a, b\},\{b, c\},\{b, d\},\{a, b, c\},\{a, b, d\},\{b, c, d\},\{a, b, c, d\}\}$ & 7 \\
\hline F114 & $\{\{a, b\},\{a, c\},\{a, d\},\{a, b, c\},\{a, b, d\},\{a, c, d\},\{a, b, c, d\}\}$ & 7 \\
\hline F115 & $\{\{b, d\},\{c, d\},\{a, b, d\},\{a, c, d\},\{b, c, d\},\{a, b, c, d\}\}$ & 6 \\
\hline F116 & $\{\{\mathrm{b}, \mathrm{c}\},\{\mathrm{c}, \mathrm{d}\},\{\mathrm{a}, \mathrm{b}, \mathrm{c}\},\{\mathrm{a}, \mathrm{c}, \mathrm{d}\},\{\mathrm{b}, \mathrm{c}, \mathrm{d}\},\{\mathrm{a}, \mathrm{b}, \mathrm{c}, \mathrm{d}\}\}$ & 6 \\
\hline
\end{tabular}




\begin{tabular}{|c|c|c|}
\hline F117 & $\{\{b, c\},\{b, d\},\{a, b, c\},\{a, b, d\},\{b, c, d\},\{a, b, c, d\}\}$ & 6 \\
\hline F118 & $\{\{a, d\},\{c, d\},\{a, b, d\},\{a, c, d\},\{b, c, d\},\{a, b, c, d\}\}$ & 6 \\
\hline F119 & $\{\{a, d\},\{b, d\},\{a, b, d\},\{a, c, d\},\{b, c, d\},\{a, b, c, d\}\}$ & 6 \\
\hline F120 & $\{\{\mathrm{a}, \mathrm{c}\},\{\mathrm{c}, \mathrm{d}\},\{\mathrm{a}, \mathrm{b}, \mathrm{c}\},\{\mathrm{a}, \mathrm{c}, \mathrm{d}\},\{\mathrm{b}, \mathrm{c}, \mathrm{d}\},\{\mathrm{a}, \mathrm{b}, \mathrm{c}, \mathrm{d}\}\}$ & 6 \\
\hline F121 & $\{\{\mathrm{c}, \mathrm{d}\},\{\mathrm{a}, \mathrm{b}, \mathrm{c}\},\{\mathrm{a}, \mathrm{b}, \mathrm{d}\},\{\mathrm{a}, \mathrm{c}, \mathrm{d}\},\{\mathrm{b}, \mathrm{c}, \mathrm{d}\},\{\mathrm{a}, \mathrm{b}, \mathrm{c}, \mathrm{d}\}\}$ & 6 \\
\hline F122 & $\{\{b, d\},\{a, b, c\},\{a, b, d\},\{a, c, d\},\{b, c, d\},\{a, b, c, d\}\}$ & 6 \\
\hline F123 & $\{\{\mathrm{b}, \mathrm{c}\},\{\mathrm{a}, \mathrm{b}, \mathrm{c}\},\{\mathrm{a}, \mathrm{b}, \mathrm{d}\},\{\mathrm{a}, \mathrm{c}, \mathrm{d}\},\{\mathrm{b}, \mathrm{c}, \mathrm{d}\},\{\mathrm{a}, \mathrm{b}, \mathrm{c}, \mathrm{d}\}\}$ & 6 \\
\hline F124 & $\{\{a, d\},\{a, b, c\},\{a, b, d\},\{a, c, d\},\{b, c, d\},\{a, b, c, d\}\}$ & 6 \\
\hline F125 & $\{\{a, c\},\{a, b, c\},\{a, b, d\},\{a, c, d\},\{b, c, d\},\{a, b, c, d\}\}$ & 6 \\
\hline F126 & $\{\{a, b\},\{a, b, c\},\{a, b, d\},\{a, c, d\},\{b, c, d\},\{a, b, c, d\}\}$ & 6 \\
\hline F127 & $\{\{a, c\},\{b, c\},\{a, b, c\},\{a, c, d\},\{b, c, d\},\{a, b, c, d\}\}$ & 6 \\
\hline F128 & $\{\{a, c\},\{a, d\},\{a, b, c\},\{a, b, d\},\{a, c, d\},\{a, b, c, d\}\}$ & 6 \\
\hline F129 & $\{\{a, b\},\{b, d\},\{a, b, c\},\{a, b, d\},\{b, c, d\},\{a, b, c, d\}\}$ & 6 \\
\hline F130 & $\{\{a, b\},\{b, c\},\{a, b, c\},\{a, b, d\},\{b, c, d\},\{a, b, c, d\}\}$ & 6 \\
\hline F131 & $\{\{a, b\},\{a, d\},\{a, b, c\},\{a, b, d\},\{a, c, d\},\{a, b, c, d\}\}$ & 6 \\
\hline F132 & $\{\{a, b\},\{a, c\},\{a, b, c\},\{a, b, d\},\{a, c, d\},\{a, b, c, d\}\}$ & 6 \\
\hline F133 & $\{\{b, d\},\{a, b, d\},\{a, c, d\},\{b, c, d\},\{a, b, c, d\}\}$ & 5 \\
\hline F134 & $\{\{c, d\},\{a, b, d\},\{a, c, d\},\{b, c, d\},\{a, b, c, d\}\}$ & 5 \\
\hline F135 & $\{\{c, d\},\{a, b, c\},\{a, c, d\},\{b, c, d\},\{a, b, c, d\}\}$ & 5 \\
\hline F136 & $\{\{\mathrm{b}, \mathrm{d}\},\{\mathrm{a}, \mathrm{b}, \mathrm{c}\},\{\mathrm{a}, \mathrm{b}, \mathrm{d}\},\{\mathrm{b}, \mathrm{c}, \mathrm{d}\},\{\mathrm{a}, \mathrm{b}, \mathrm{c}, \mathrm{d}\}\}$ & 5 \\
\hline F137 & $\{\{b, c\},\{a, b, c\},\{a, c, d\},\{b, c, d\},\{a, b, c, d\}\}$ & 5 \\
\hline F138 & $\{\{b, c\},\{a, b, c\},\{a, b, d\},\{b, c, d\},\{a, b, c, d\}\}$ & 5 \\
\hline F139 & $\{\{\mathrm{a}, \mathrm{b}, \mathrm{c}\},\{\mathrm{a}, \mathrm{b}, \mathrm{d}\},\{\mathrm{a}, \mathrm{c}, \mathrm{d}\},\{\mathrm{b}, \mathrm{c}, \mathrm{d}\},\{\mathrm{a}, \mathrm{b}, \mathrm{c}, \mathrm{d}\}\}$ & 5 \\
\hline F140 & $\{\{\mathrm{a}, \mathrm{d}\},\{\mathrm{a}, \mathrm{b}, \mathrm{d}\},\{\mathrm{a}, \mathrm{c}, \mathrm{d}\},\{\mathrm{b}, \mathrm{c}, \mathrm{d}\},\{\mathrm{a}, \mathrm{b}, \mathrm{c}, \mathrm{d}\}\}$ & 5 \\
\hline F141 & $\{\{a, d\},\{a, b, c\},\{a, b, d\},\{a, c, d\},\{a, b, c, d\}\}$ & 5 \\
\hline F142 & $\{\{a, c\},\{a, b, c\},\{a, c, d\},\{b, c, d\},\{a, b, c, d\}\}$ & 5 \\
\hline F143 & $\{\{a, c\},\{a, b, c\},\{a, b, d\},\{a, c, d\},\{a, b, c, d\}\}$ & 5 \\
\hline F144 & $\{\{a, b\},\{a, b, c\},\{a, b, d\},\{b, c, d\},\{a, b, c, d\}\}$ & 5 \\
\hline F145 & $\{\{a, b\},\{a, b, c\},\{a, b, d\},\{a, c, d\},\{a, b, c, d\}\}$ & 5 \\
\hline F146 & $\{\{\mathrm{b}, \mathrm{d}\},\{\mathrm{a}, \mathrm{b}, \mathrm{d}\},\{\mathrm{b}, \mathrm{c}, \mathrm{d}\},\{\mathrm{a}, \mathrm{b}, \mathrm{c}, \mathrm{d}\}\}$ & 4 \\
\hline F147 & $\{\{c, d\},\{a, c, d\},\{b, c, d\},\{a, b, c, d\}\}$ & 4 \\
\hline F148 & $\{\{\mathrm{b}, \mathrm{c}\},\{\mathrm{a}, \mathrm{b}, \mathrm{c}\},\{\mathrm{b}, \mathrm{c}, \mathrm{d}\},\{\mathrm{a}, \mathrm{b}, \mathrm{c}, \mathrm{d}\}\}$ & 4 \\
\hline F149 & $\{\{a, b, c\},\{a, b, d\},\{a, c, d\},\{a, b, c, d\}\}$ & 4 \\
\hline F150 & $\{\{a, b, d\},\{a, c, d\},\{b, c, d\},\{a, b, c, d\}\}$ & 4 \\
\hline F151 & $\{\{\mathrm{a}, \mathrm{b}, \mathrm{c}\},\{\mathrm{a}, \mathrm{c}, \mathrm{d}\},\{\mathrm{b}, \mathrm{c}, \mathrm{d}\},\{\mathrm{a}, \mathrm{b}, \mathrm{c}, \mathrm{d}\}\}$ & 4 \\
\hline F152 & $\{\{a, b, c\},\{a, b, d\},\{b, c, d\},\{a, b, c, d\}\}$ & 4 \\
\hline F153 & $\{\{a, d\},\{a, b, d\},\{a, c, d\},\{a, b, c, d\}\}$ & 4 \\
\hline F154 & $\{\{a, c\},\{a, b, c\},\{a, c, d\},\{a, b, c, d\}\}$ & 4 \\
\hline F155 & $\{\{a, b\},\{a, b, c\},\{a, b, d\},\{a, b, c, d\}\}$ & 4 \\
\hline F156 & $\{\{a, c, d\},\{b, c, d\},\{a, b, c, d\}\}$ & 3 \\
\hline F157 & $\{\{a, b, d\},\{a, c, d\},\{a, b, c, d\}\}$ & 3 \\
\hline F158 & $\{\{a, b, d\},\{b, c, d\},\{a, b, c, d\}\}$ & 3 \\
\hline F159 & $\{\{a, b, c\},\{a, c, d\},\{a, b, c, d\}\}$ & 3 \\
\hline F160 & $\{\{a, b, c\},\{b, c, d\},\{a, b, c, d\}\}$ & 3 \\
\hline F161 & $\{\{a, b, c\},\{a, b, d\},\{a, b, c, d\}\}$ & 3 \\
\hline F162 & $\{\{a, c, d\},\{a, b, c, d\}\}$ & 2 \\
\hline F163 & $\{\{b, c, d\},\{a, b, c, d\}\}$ & 2 \\
\hline F164 & $\{\{a, b, d\},\{a, b, c, d\}\}$ & 2 \\
\hline F165 & $\{\{a, b, c\},\{a, b, c, d\}\}$ & 2 \\
\hline F166 & $(\{a, b, c, d\}\}$ & 1 \\
\hline
\end{tabular}

The lattice $<\Phi, \subseteq>$ is constructed as given in Fig. 3 whose elements are 166 topological filters defined over the ground set $X=\{a, b, c, d\}$. Note that the symbol $\subseteq$ denotes the partial order relation of 'subset of'. One can enumerate 13,767 linear maximal filter chains from this lattice. One can compute STRT spectra for all the 13,767 linear maximal filter chains. For example, one linear maximal filter chain is considered here and its STRT shown in table 3. 
Table 3: Filter chain and its STRT spectrum

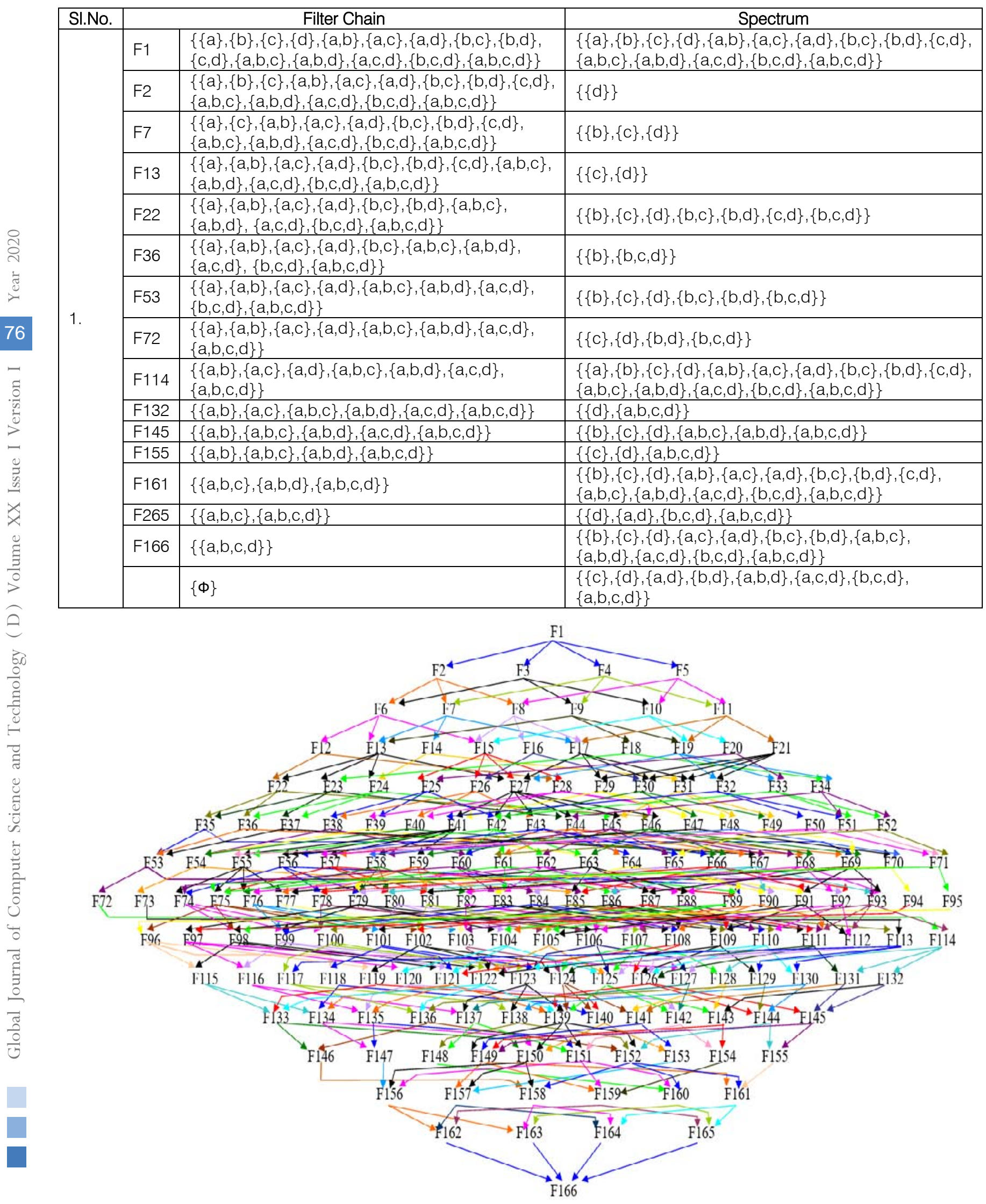

Fig. 3: Lattice diagram showing the linear filter chains over a set $X=\{a, b, c, d\}$ 


\section{OBSERVATIONS}

By applying STRT to the above maximal filter chains, we examined few pair-wise intersection properties. The level with filter of maximum cardinality is considered as Level1.By taking two random filter chains, which deviate at certain levels the following properties were observed:

Deviation in any combination of even levels results in following properties:

Union of spectra of two filter chains is same as Spectrum of Intersection of those two filter chains.

Intersection of spectra of two filter chains is same as Spectrum of Union of those two filter chains.

One can easily verify these properties by applying STRT to the below pair of filter chains:

For example, let us consider $n=3$,

Deviation in Level 2-

$$
\begin{aligned}
& \text { F1-F2-F5-F8-F12-F15-F18 } \\
& \text { F1-F3-F5-F8-F12-F15-F18 }
\end{aligned}
$$

Deviation in Level 4-

$$
\begin{aligned}
& \text { F1-F3-F5-F8-F12-F15-F18 } \\
& \text { F1-F3-F5-F11-F12-F15-F18 }
\end{aligned}
$$

Deviation in Level 6-

$$
\begin{aligned}
& \text { F1-F4-F7-F10-F14-F16-F18 } \\
& \text { F1-F4-F7-F10-F14-F17-F18 }
\end{aligned}
$$

Deviation in Level 4 and 6-

$$
\begin{aligned}
& \text { F1-F2-F5-F8-F12-F15-F18 } \\
& \text { F1-F2-F5-F11-F12-F16-F18 }
\end{aligned}
$$

Deviation in any combination of odd levels results in following properties:

Union of spectra of two filter chains is same as Spectrum of Union of those two filter chains.

Intersection of spectra of two filter chains is same as Spectrum of Intersection of those two filter chains.

One can easily verify these properties by applying STRT to the below pair of filter chains:

Deviation in Level 3-

$$
\begin{aligned}
& \text { F1-F3-F5-F11-F12-F15-F18 } \\
& \text { F1-F3-F7-F11-F12-F15-F18 }
\end{aligned}
$$

Deviation in Level 5-

$$
\begin{aligned}
& \text { F1-F2-F5-F11-F12-F16-F18 } \\
& \text { F1-F2-F5-F11-F14-F16-F18 }
\end{aligned}
$$

Deviation in Level 3 and 5-

F1-F3-F5-F11-F12-F15-F18

F1-F3-F7-F11-F13-F15-F18

\section{Concluding Remarks}

All orthogonal transforms, be it continuous or discrete, are models of first order logic, that is, they have been developed in the framework of first order logic that deal with elements of sets. Alternatively, STRT is a novel concept developed in the framework of second order logic that deals with set of sets, and so it has potential applications to solve problems related to functions of sets.

\section{ACKNOWLEDGEMENT}

The first author thanks the second author Professor E. G. Rajan for his valuable guidance and encouragement to work on the concept introduced by him in the year 1997. Thanks are due to the management of the University of Petroleum and Energy Studies, Dehradun, India for encouraging us to carry out this in-depth research. The authors express their appreciation to Mr. Sathya Govindarajan and Mrs. Prashanthi Govindarajan, Directors, Pentagram Research Centre Private Limited, Hyderabad, India who have developed many programs to test our concepts and participated untiringly in our ongoing research.

\section{References Références Referencias}

1. Rajan, E. G, On the Notion of Generalized Rapid Transformation, World multi conference on Systemics, Cybernetics and Informatics, Caracas, Venezuela, July $7-11,1997$. 